\title{
WATER-LEVEL DATA FROM WELLS IN THE VICINITY OF THE WASTE ISOLATION PILOT PLANT, SOUTHEASTERN NEW MEXICO \\ By Steven F. Richey
}

U.S. GEOLOGICAL SURVEY

Open-File Report 87-120

Prepared in cooperation with the

U.S. DEPARTMENT OF ENERGY

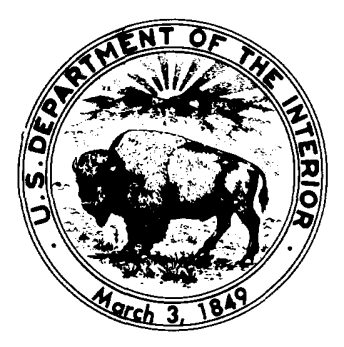

Albuquerque, New Mexico 
DEPARTMENT OF THE INTERIOR

DONALD PAUL HODEL, Secretary

U.S. GEOLOGICAL SURVEY

Dallas L. Peck, Director

For additional information

write to:

District Chief

U.S. Geological Survey

Water Resources Division

Pinetree office Park

4501 Indian School Rd. NE, Suite 200

Albuquerque, New Mexico 87110
Copies of this report can

be purchased from:

U.S. Geological Survey

Books and Open-File Reports Section Federal Center

Box 25425

Denver, Colorado 80225 


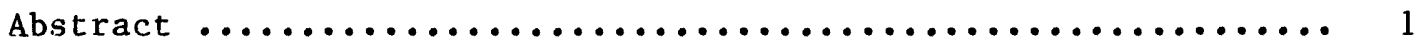

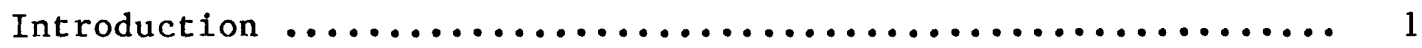

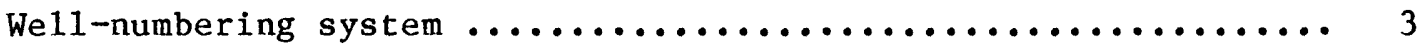

Measurement methods $\ldots \ldots \ldots \ldots \ldots \ldots \ldots \ldots \ldots \ldots \ldots \ldots \ldots \ldots \ldots \ldots$

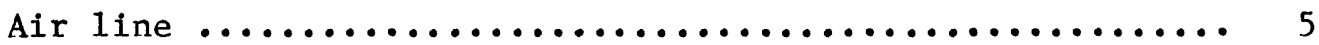

Lynes Pressure Sentry System ..................... 5

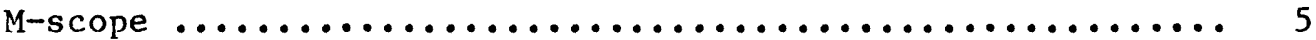

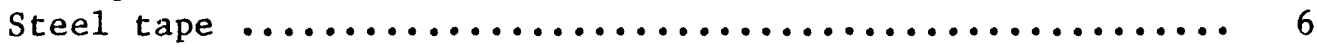

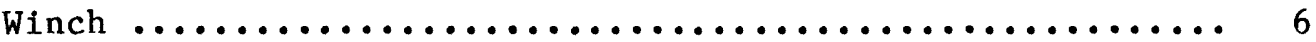

FIGURES

Figure 1. Map showing general location of the Waste Isolation Pilot Plant (WIPP) and wells in which water levels were measured .............. 2

2. Diagran showing the system of numbering wells

\section{TABLES}

Tables 1-8. Water-level records for:

1. We11 AEC-8, lower Delaware sand (economic usage) of the Bell Canyon Formation ...... 7

2. We11 AEC-8, upper Delaware sand (economic usage) of the Bell Canyon Formation ...... 9

3. Well H-1, Magenta Dolomite Member of the

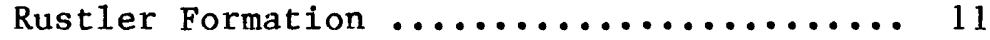

4. Well H-1, Culebra Dolomite Member of the

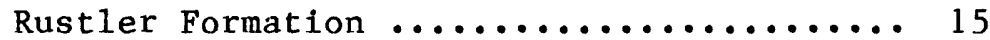

5. Well $\mathrm{H}-1$, Magenta Dolomite and Culebra Dolomite Members of the Rustier Formation ........................ 19

6. We11 H-2A, Magenta Dolomite Member of the Rustler Formation .................. 20

7. Well H-2B, Magenta Dolomite Member of the Rustler Formation ....................... 24

8. Well H-2B, Culebra Dolomite Member of the

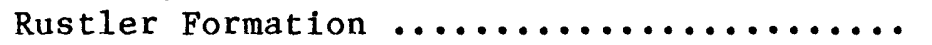


Tables 9-29. Water-level records for:

9. We11 H-2B, Magenta Dolomite and Culebra Dolomite Members of the Rustler Formation ....................... 27

10. Well H-2C, Culebra Dolomite Member of the Rustler Formation ...................... 30

11. Well H-2C, Rustler Formation-Salado Formation contact zone ................ 34

12. Well $\mathrm{H}-3$, Magenta Dolomite Member of the

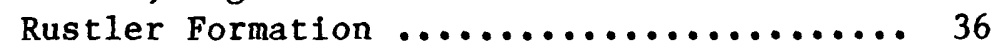

13. Well $\mathrm{H}-3$, Culebra Dolomite Member of the Rustler Formation ................. 40

14. Well H-3, Magenta Dolomite and Culebra Dolomite Members of the Rustler Formation ........................ 44

15. Well H-4A, Magenta Dolomite Member of the Rustler Formation ................. 45

16. Well H-4B, Culebra Dolomite Member of the Rustler Formation ................. 47

17. Well H-4C, Rustler Formation-Salado

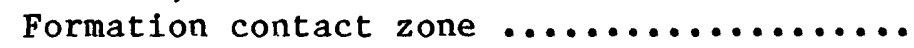

18. Well H-5A, Magenta Dolomite Member of the Rustler Formation ................. 50

19. Well H-5B, Culebra Dolomite Member of the Rustler Formation ................. 51

20. Well H-5C, Rustler Formation-Salado Formation contact zone .............. 53

21. We11 H-6A, Magenta Dolomite Member of the Rustler Formation .................. 54

22. Well H-6B, Culebra Dolomite Member of the Rustler Formation ................. 55

23. Well H-6C, Rustler Formation-Salado Formation contact zone .............. 57

24. Well H-7A, Magenta Dolomite Member of the Rustler Formation ................. 58

25. Well H-7B, Culebra Dolomite Member of the Rustler Formation .................... 59

26. Well H-7C, Rustler Formation-Salado Formation contact zone ............... 61

27. Well H-8A, Magenta Dolomite Member of the Rustler Formation ................. 62

28. Well H-8B, Culebra Dolomite Member of the Rustler Formation ................. 63

29. Well H-8C, Rustler Formation-Salado Formation contact zone ............... 
Tables 30-51. Water-level records for:

30. Well H-9A, Magenta Dolomite Member of the Rustler Formation ..................... 66

31. Well H-9B, Culebra Dolomite Member of the Rustler Formation ................. 68

32. We11 H-9C, Rustler Formation-Salado Formation contact zone ............... 70

33. Well H-10A, Magenta Dolomite Member of the Rustler Formation .................... 71

34. Well H-10B, Culebra Dolomite Member of the Rustler Formation ................. 73

35. We11 H-10C, Rustler Formation-Salado Formation contact zone ............... 75

36. Well P-14, Culebra Dolomite Member of the Rustler Formation ................ 76

37. Well P-14, Rustler Formation-Salado Formation contact zone .............. 78

38. Well P-15, Culebra Dolomite Member of the Rustler Formation ................. 80

39. Wel1 P-15, Rustler Formation-Salado Formation contact zone .............. 82

40. Well P-17, Culebra Dolomite Member of the Rustler Formation .................. 84

41. Well P-17, Rustler Formation-Salado Formation contact zone ................. 85

42. Wel1 P-18, Culebra Dolomite Member of the Rustler Formation ................ 86

43. Well P-18, Rustler Formation-Salado Formation contact zone .............. 88

44. Well WIPP-16, breccia ................. 90

45. We11 WIPP-25, Magenta Dolomite Member of the Rustler Formation .................... 91

46. Well WIPP-25, Culebra Dolomite Member of the Rustler Formation .................. 92

47. We11 WIPP-25, Rustler Formation-Salado Formation contact zone .................93 93

48. Well WIPP-26, Magenta Dolomite Member of the Rustler Formation ...................... 94

49. Well WIPP-26, Culebra Dolomite Member of the Rustler Formation ..................... 95

50. Wel1 WIPP-26, Rustler Formation-Salado Formation contact zone .............. 96

51. Well WIPP-27, Magenta Dolomite Member of the Rustler Formation ................ 97 
Tables 52-61. Water-1eve1 records for:

52. We1l WIPP-27, Culebra Dolomite Member of the Rustler Formation ................. 98

53. We1l WIPP-27, Rustler Formation-Salado Formation contact zone ............... 99

54. We1l WIPP-28, Magenta Dolomite Member of the

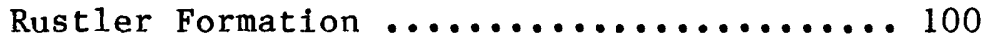

55. Well WIPP-28, Culebra Dolomite Member of the

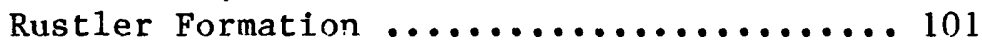

56. Well WIPP-28, Rustler Formation-Salado

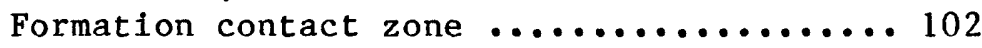

57. Well WIPP-29, Culebra Dolomite Member of the

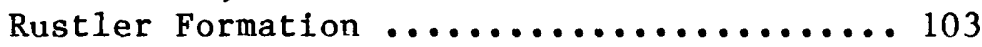

58. Well WIPP-29, Rustler Formation-Salado Formation contact zone ................... 104

59. Well WIPP-30, Magenta Dolomite Member of the

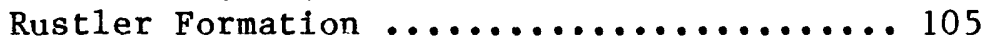

60. Wel1 WIPP-30, Culebra Dolomite Member of the

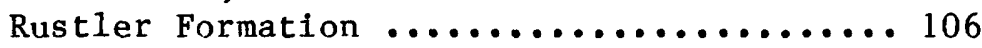

61. We1l WIPP-30, Rustler Formation-Salado Formation contact zone ...............107

\author{
CONVERSION FACTORS
}

In this report, figures for measurements are given in inch-pound units only. The following table contains factors for converting to metric units.

$\begin{array}{lcl}\text { Multiply inch-pound units } & \text { By } & \text { To obtain m } \\ \text { inch } & 25.40 & \text { millimeter } \\ \text { foot } & 0.3048 & \text { meter } \\ \text { gallon } & 3.785 & \text { liter }\end{array}$

The use of brand names in this report is for identification purposes only and does not constitute endorsement by the U.S. Geological Survey.

Sea level: In this report, "sea level" refers to the National Geodetic Vertical Datum of 1929 (NGVD of 1929)-- a geodetic datum derived from a general adjustment of the first-order level nets of both the United States and Canada, formerly called "Mean Sea Level of 1929." 


\author{
WATER-LEVEL DATA FROM WELLS IN THE VICINITY OF \\ THE WASTE ISOLATION PILOT PLANT, \\ SOUTHEASTERN NEW MEXICO \\ By Steven F. Richey
}

\begin{abstract}
The U.S. Geological Survey monitored water levels in wells in the vicinity of the Waste Isolation Pilot Plant in southeastern New Mexico during 1977 to 1985. Water-level data are presented, as are brief descriptions of measurement methods used.
\end{abstract}

\title{
INTRODUCTION
}

The U.S. Geological Survey, at the request of the U.S. Department of Energy, is investigating the geohydrology of the area in the vicinity of the Waste Isolation Pilot Plant (WIPP) in southeastern New Mexico (fig. 1). The WIPP is a storage facility constructed in bedded salts of the Permian Salado Formation in which defense-associated transuranic wastes will be deposited. Water levels in three primary water-bearing zones were monitored: the Magenta Dolomite Member of the Rustler Formation, the Culebra Dolomite Member of the Rustler Formation, and the Rustler Formation-Salado Formation contact zone. In addition, water levels in the upper and lower Delaware sands (economic usage) were monitored in one well. Water-level measurements in this report do not necessarily reflect static conditions because perturbations to the water-bearing zones caused by well completion and testing, particularly in the Rustler-Salado contact zone, may take months or years to return to normal. This report presents water-level data collected during monitoring conducted from 1977 to 1985 (tables 1 through 61). 


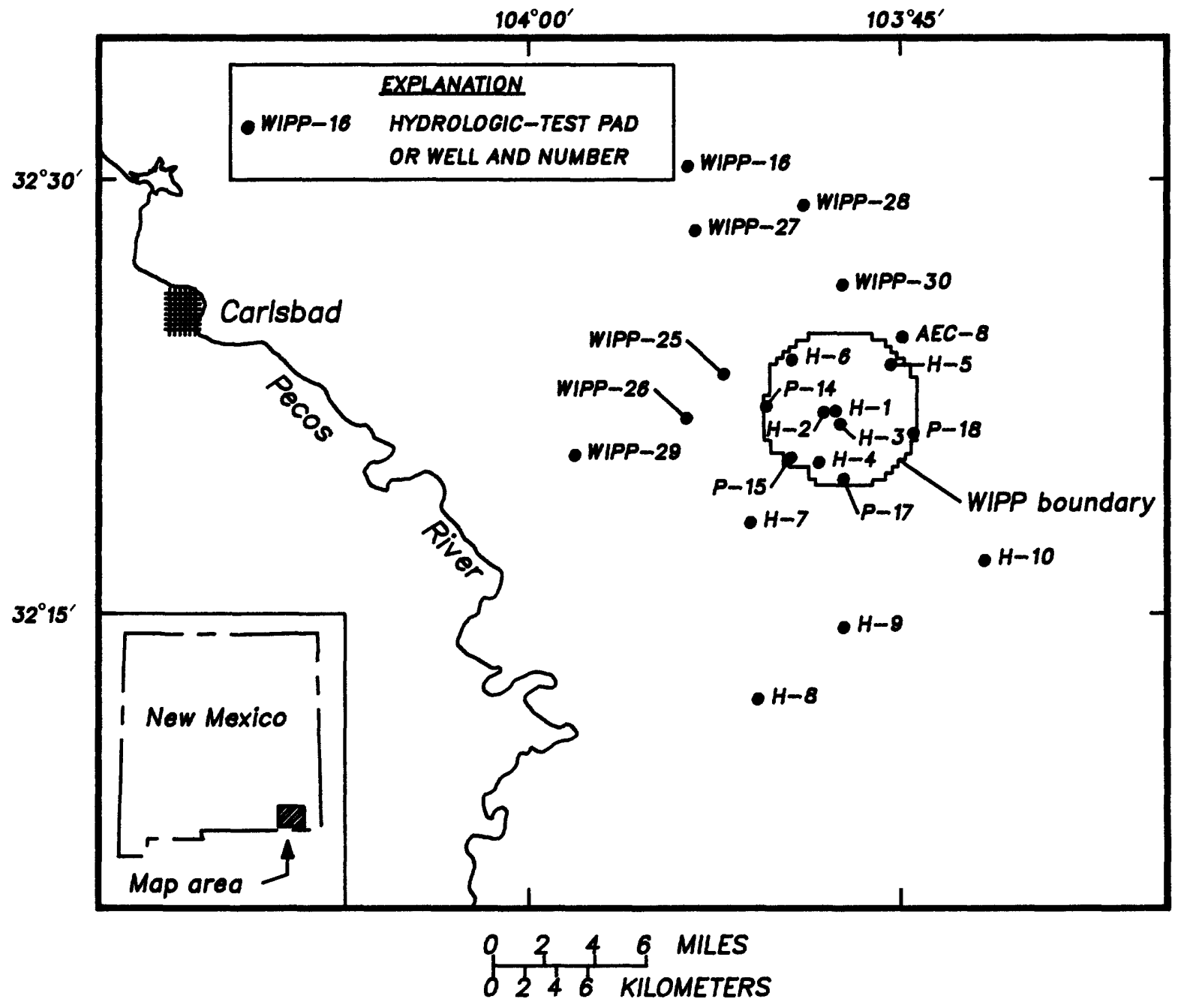

Figure 1. -- General location of the Waste lsolation Pilot Plant (WIPP) and wells in which water levels were measured. 
Several series of wells were monitored. AEC-8 is one of a series of wells originally drilled to obtain 1ithologic and stratigraphic data. AEC-8 was later deepened and completed to enable monitoring of water-bearing zones. The "H" series was drilled specifically to obtain geohydrologic data in the vicinity of WIPP. With the exception of wells $\mathrm{H}-1$ and $\mathrm{H}-3$, " $\mathrm{H}$ " series wells were drilled in sets of three at a pad. Each well at a pad was completed in a different zone of interest: well "A" was completed in the Magenta Dolomite Member of the Rustler Formation, well "B" was completed in the Culebra Dolomite Member of the Rustler Formation, and well " $\mathrm{C}$ " was completed in the Rustler Formation-Salado Formation contact zone. Exceptions are H-2B, which was completed in both the Magenta Dolomite and the Culebra Dolomite Members of the Rustler Formation, and $\mathrm{H}-2 \mathrm{C}$, completed in both the Culebra Dolomite Member of the Rustler Formation and the Rustler Formation-Salado Formation contact zone. At a given pad, these wells generally are 50 to 100 feet apart. The "WIPP" series of wells consists of single wells drilled to obtain geologic data. They were secondarily completed for hydrologic testing and monitoring, with each well completed in all three zones of interest where they were present. The "P" series of wells consists of single wells drilled to define the extent of potash deposits in the vicinity of WIPP. They were secondarily completed for hydrologic testing and monitoring of the Culebra Dolomite Member of the Rustler Formation and the Rustler Formation-Salado Formation contact zone.

\section{WELL-NUMBERING SYSTEM}

The system of numbering wells in this report is based on the common subdivision of public lands into sections. The well number consists of five segments separated by periods (fig. 2). The first segment is the township number, the second segment is the range number, and the third segment is the section number. Because all township blocks in the vicinity of the WIPP are south of the New Mexico Base Line and east of the New Mexico Principal Meridian, the letters " $S$ " and " $E$ " indicating direction are not used in this report. Hence, the number 22.31.11 is assigned to any well located in sec. $11, \mathrm{~T} .22 \mathrm{~S} ., \mathrm{R} .31 \mathrm{E}$. The fourth and fifth segments of the well number represent the number of feet from the indicated section line to the well. For example, "0935FNL.1979FWL" describes a well that is 935 feet along a perpendicular extended from the north section line and 1,979 feet along a perpendicular extended from the west section line. 


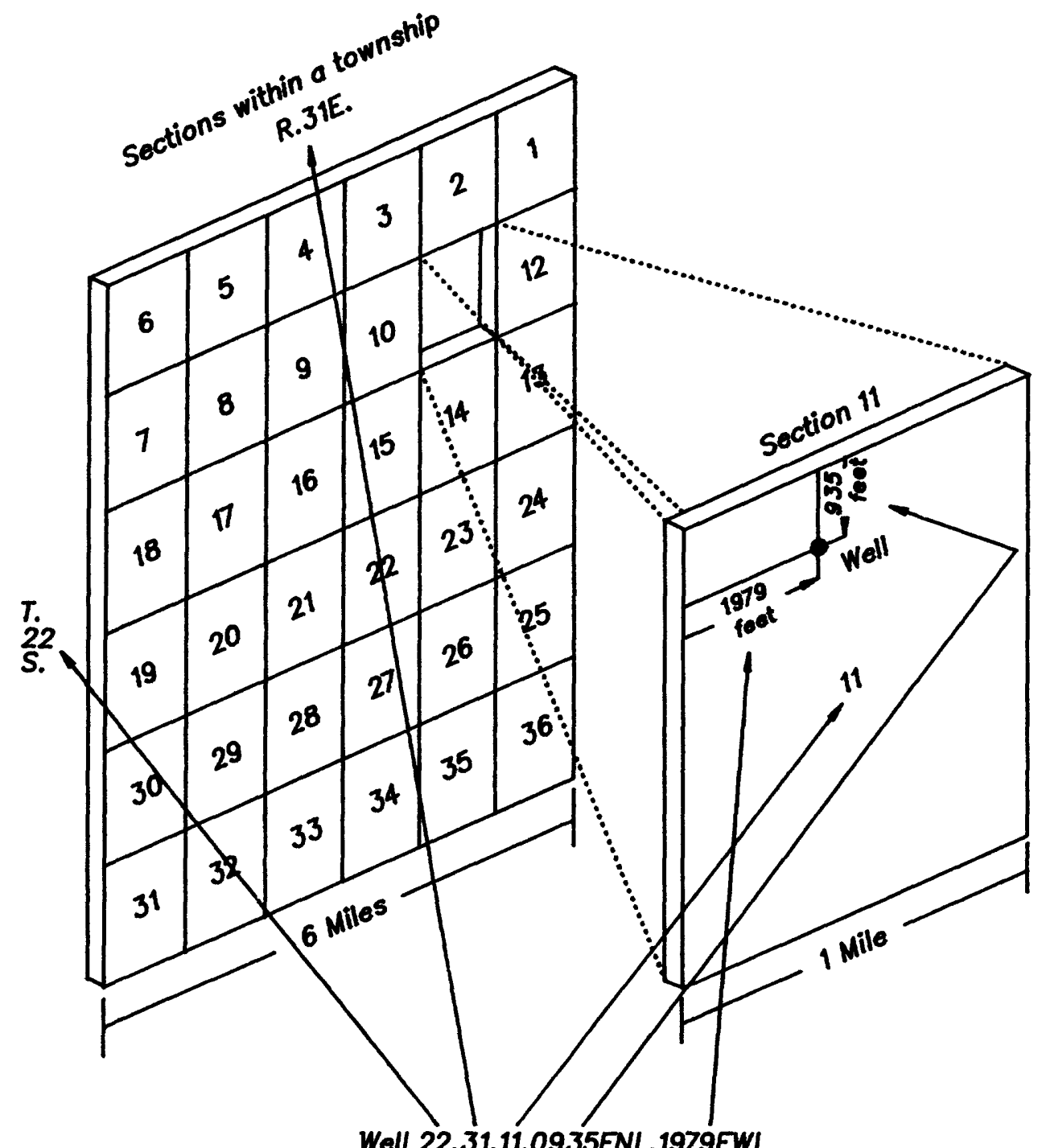

Well 22.31.11.0935FNL.1979FWL

Figure 2. --System of numbering wells in this report. 
A variety of methods was used to measure water levels. The particular method utilized at a given time depended on several factors, including the amount of condensation in the well, well-head configuration, depth to water, rate of water-1evel change, and availability of equipment.

\section{Air Line}

An air-line measurement system was implemented by installing tubing in the well to below the water level. A pressure transducer was connected to the tubing near the well head. The pressure that the transducer sensed was converted to freshwater head. The freshwater head was then subtracted from the length of air line to give the depth to water. Air-line measurements are not corrected for fluid density and are reported to the nearest foot.

\section{Lynes Pressure Sentry System}

A Lynes Pressure Sentry System was installed by placing a Lynes-Sentry pressure probe downhole below the water level. The pressure at the probe was converted to feet of freshwater head. The freshwater head was then subtracted from the depth of the probe to determine the depth to water. Lynes Pressure Sentry System measurements are not corrected for fluid density and are reported to the nearest foot.

\section{M-Scope}

The M-scope is a device consisting of a spool of two-conductor wire, a meter, and a battery. A probe on the end of the wire completes an electrical circuit when it is submerged, causing a deflection of the meter. Depth to water was obtained by determining the length of wire needed for the probe to make contact with the water in the well. M-scope measurements are reported to the nearest foot. 


\section{$\underline{\text { Steel Tape }}$}

Steel-tape measurements were performed using a reel containing calibrated steel tape. The tape was lowered down the well until the first few feet were submerged, and the footage was recorded at the measuring point at the surface. The tape was then raised to the surface where the length of wet tape was recorded. This wet-tape footage was subtracted from the footage held at the measuring point to get the depth below the measuring point, which was then corrected to land-surface datum. Steel-tape measurements are reported to the nearest 0.1 foot.

\section{Winch}

Winch measurements were performed by lowering a transducer on the end of a logging cable down the well until it contacted the water surface. The footage was then read of $f$ of a digital footage counter on the winch. Winch measurements are reported to the nearest foot. 
Table 1. Water-level records for well AEC-8, lower Delaware sand (economic usage) of the Bell Canyon Formation

[Measurement code: A, Air-line measurement; L, Lynes Pressure Sentry System; M, M-Scope; S, Steel tape; W, Winch. Total depth is in feet below land surface. Land-surface altitude is in feet above sea level.]

Location: $22.31 \cdot 11.0935 \mathrm{FNL} .1979 \mathrm{FWL}$

Total depth: $\quad 4,910.0$ Land-surface altitude: $\quad 3,531.5$

\begin{tabular}{ll} 
Water level, \\
in feet below \\
Date & land surface Measurement code and remarks \\
\hline
\end{tabular}

\begin{tabular}{|c|c|}
\hline $\begin{array}{r}11 / 4 / 77 \\
11 / 30 / 77 \\
1 / 8 / 78 \\
2 / 3 / 78 \\
3 / 2 / 78\end{array}$ & $\begin{array}{l}687 \\
615 \\
591 \\
584 \\
580\end{array}$ \\
\hline $\begin{array}{ll}4 / & 4 / 78 \\
5 / & 3 / 78 \\
6 / & 1 / 78 \\
7 / & 6 / 78 \\
8 / & 1 / 78\end{array}$ & $\begin{array}{l}577 \\
574 \\
573 \\
572 \\
570\end{array}$ \\
\hline $\begin{array}{r}9 / 6 / 78 \\
10 / 3 / 78 \\
11 / 8 / 78 \\
12 / 6 / 78 \\
1 / 16 / 79\end{array}$ & $\begin{array}{l}570 \\
570 \\
570 \\
570 \\
568\end{array}$ \\
\hline $\begin{array}{l}2 / 11 / 79 \\
3 / 5 / 79 \\
6 / 5 / 79 \\
7 / 10 / 79 \\
8 / 7 / 79\end{array}$ & $\begin{array}{l}568 \\
569 \\
568 \\
568 \\
568\end{array}$ \\
\hline 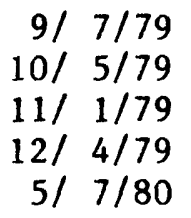 & $\begin{array}{l}567 \\
567 \\
567 \\
567 \\
567\end{array}$ \\
\hline 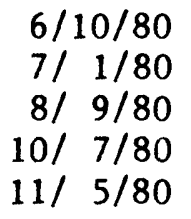 & $\begin{array}{l}567 \\
567 \\
568 \\
567 \\
568\end{array}$ \\
\hline
\end{tabular}


Table 1. Water-level records for well AEC-8, lower Delaware sand (economic usage) of the Bell Canyon Formation

- Concluded

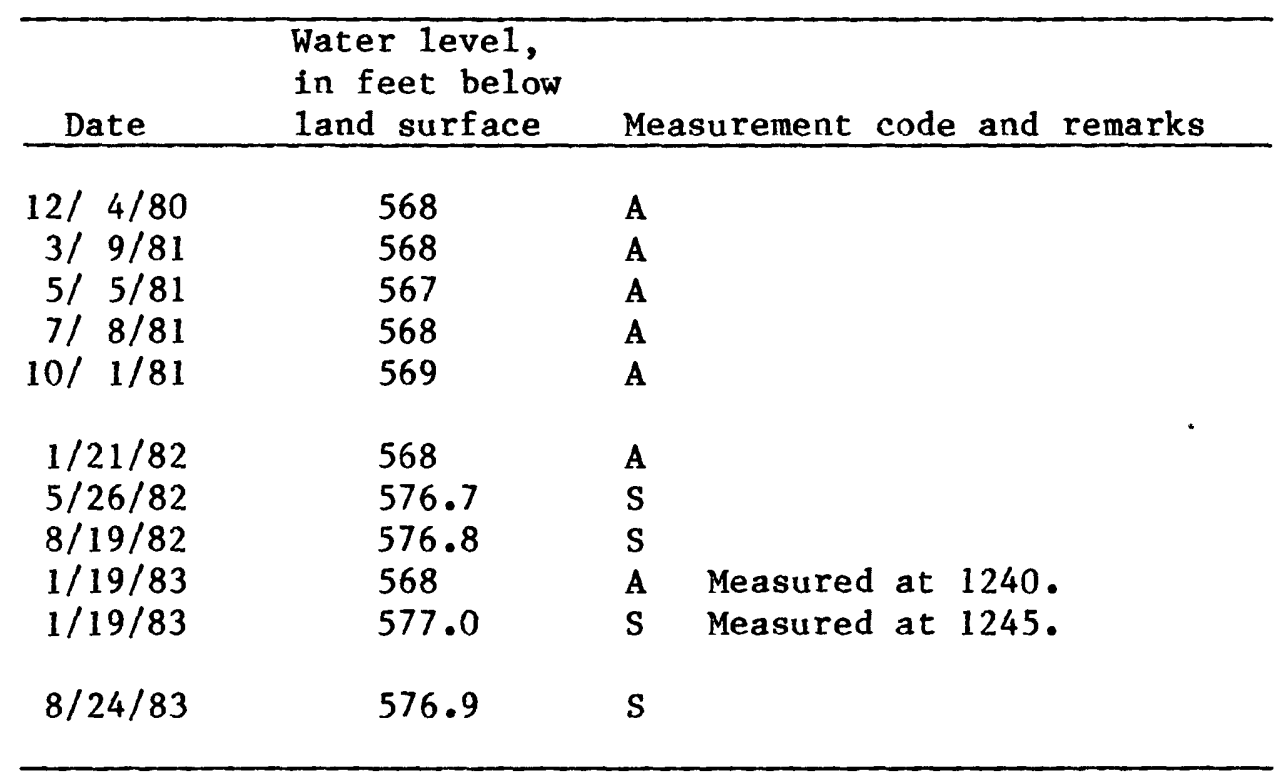


Table 2. Water-level records for well AEC-8, upper Delaware sand (economic usage) of the Bell Canyon Formation

[Measurement code: A, Air-1ine measurement; L, Lynes Pressure Sentry System; M, M-Scope; S, Steel tape; W, Winch. Total depth is in feet below land surface. Land-surface altitude is in feet above sea level.]

Location: $22.31 \cdot 11.0935 \mathrm{FNL} \cdot 1979 \mathrm{FWL}$

Total depth: $\quad 4,910.0$ Land-surface altitude: $\quad 3,531.5$

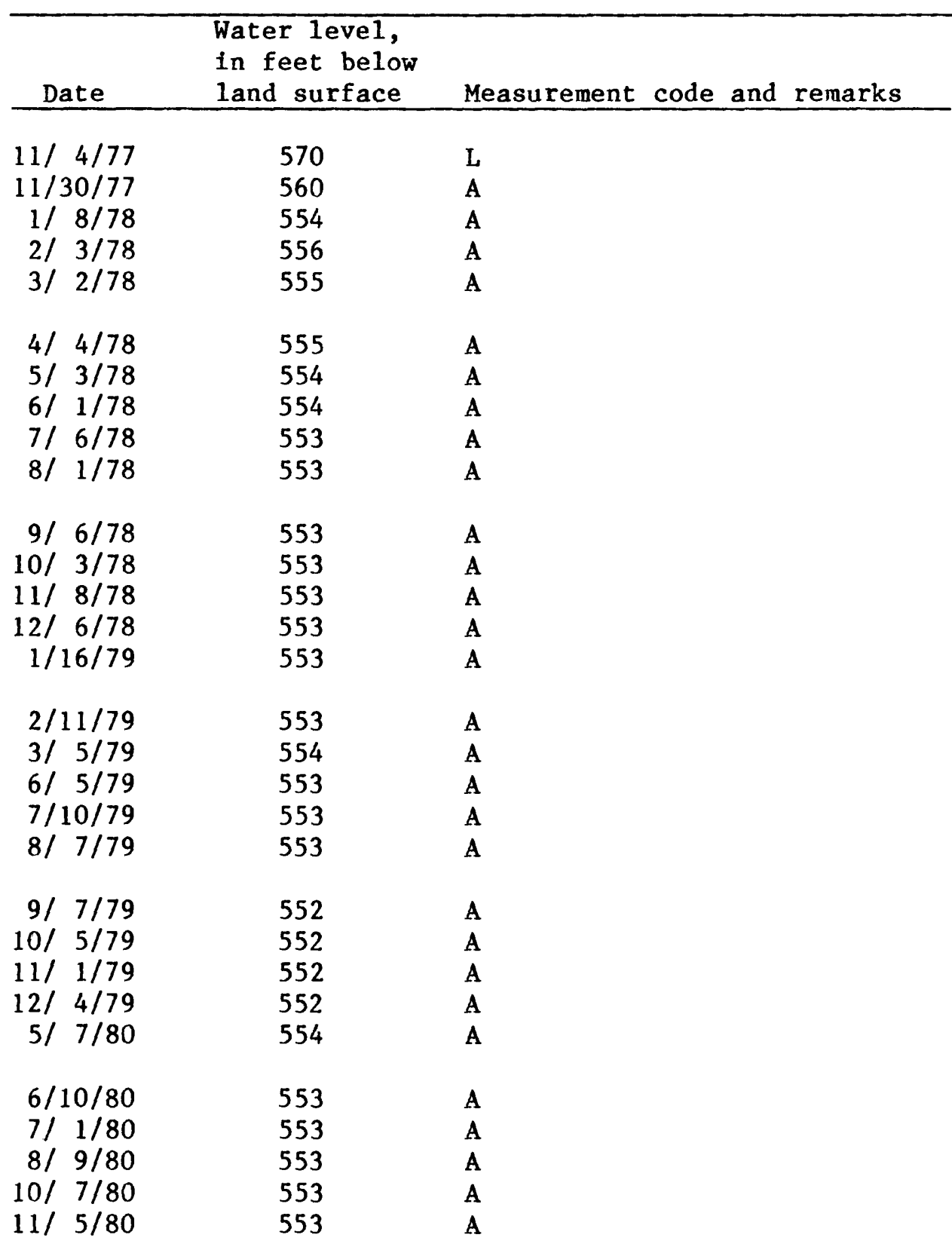


Table 2. Water-level records for well AEC-8, upper Delaware sand (economic usage) of the Bell Canyon Formation

- Concluded

\begin{tabular}{ccc}
\hline & $\begin{array}{l}\text { Water level, } \\
\text { in feet below } \\
\text { land surface }\end{array}$ & Measurement code and remarks \\
\hline $12 / 4 / 80$ & 553 & A \\
$3 / 9 / 81$ & 553 & A \\
$5 / 5 / 81$ & 553 & A \\
$7 / 8 / 81$ & 553 & A \\
$10 / 1 / 81$ & 553 & A \\
& & \\
$1 / 21 / 82$ & 553 & A \\
$5 / 26 / 82$ & 560.6 & S \\
$8 / 19 / 82$ & 553 & A \\
$1 / 19 / 83$ & 553 & A \\
\hline
\end{tabular}


Table 3. Water-level records for well $\mathrm{H}-1$, Magenta Dolomite Member of the Rustler Formation

[Measurement code: A, Afr-line measurement; L, Lynes Pressure Sentry System; M, M-Scope; S, Steel tape; W, Winch. Total depth is in feet below land surface. Land-surface altitude is in feet above sea level.]

Location: $22.31 .29 .0623 \mathrm{FNL} .1083 \mathrm{FEL}$

Total depth: $\quad 856.0$ Land-surface altitude: $\quad 3,396.8$

\begin{tabular}{ccc}
\hline & $\begin{array}{c}\text { Water level, } \\
\text { in feet below } \\
\text { land surface }\end{array}$ & Measurement code and remarks \\
Date & 342 & M \\
5/10/77 & 269.9 & S \\
$5 / 25 / 77$ & 257.3 & S \\
$6 / 23 / 77$ & 254 & M \\
$8 / 4 / 77$ & 252 & M \\
$9 / 3 / 77$ & & \\
& 249 & M \\
$10 / 3 / 77$ & 251 & M \\
$11 / 3 / 77$ & 250 & M \\
$12 / 1 / 77$ & 248 & A \\
$1 / 8 / 78$ & 250 & A \\
$2 / 3 / 78$ & & \\
$3 / 3 / 78$ & 250 & A \\
$4 / 6 / 78$ & 249.4 & S \\
$5 / 3 / 78$ & 249.2 & S \\
$6 / 2 / 78$ & 248 & A \\
$7 / 6 / 78$ & 248 & A \\
$8 / 2 / 78$ & 248 & A \\
$9 / 6 / 78$ & 248 & A \\
$10 / 3 / 78$ & 247 & A \\
$11 / 7 / 78$ & 247 & A \\
$1 / 16 / 79$ & 247 & A \\
$2 / 10 / 79$ & 247.8 & S \\
$3 / 5 / 79$ & 247.2 & S \\
$3 / 13 / 79$ & 247.0 & S \\
$10 / 6 / 80$ & 259.8 & S \\
$11 / 4 / 80$ & 258.7 & S \\
$12 / 4 / 80$ & 259.1 & S \\
$1 / 5 / 81$ & 258.5 & S \\
$2 / 4 / 81$ & 258.0 & S \\
$3 / 5 / 81$ & 257.3 & S \\
$4 / 2 / 81$ & 256.8 & S \\
& &
\end{tabular}


Table 3. Water-level records for well $\mathrm{H}-1$, Magenta Dolomite Member of the Rustler Formation - Continued

\begin{tabular}{|c|c|c|}
\hline Date & $\begin{array}{l}\text { Water level, } \\
\text { in feet below } \\
\text { land surface }\end{array}$ & Measurement code and remarks \\
\hline $\begin{array}{ll}5 / & 4 / 81 \\
6 / & 1 / 81 \\
7 / & 6 / 81 \\
7 / 23 / 81 \\
7 / 29 / 81\end{array}$ & $\begin{array}{l}256.3 \\
255.8 \\
255.4 \\
255.3 \\
255.3\end{array}$ & $\begin{array}{l}\mathrm{S} \\
\mathrm{S} \\
\mathrm{S} \\
\mathrm{S} \\
\mathrm{S}\end{array}$ \\
\hline $\begin{array}{r}8 / 3 / 81 \\
8 / 10 / 81 \\
8 / 21 / 81 \\
9 / 2 / 81 \\
10 / 6 / 81\end{array}$ & $\begin{array}{l}255.1 \\
255.1 \\
254.9 \\
254.7 \\
254.7\end{array}$ & $\begin{array}{l}\mathrm{S} \\
\mathrm{S} \\
\mathrm{S} \\
\mathrm{S} \\
\mathrm{S}\end{array}$ \\
\hline $\begin{array}{l}10 / 14 / 81 \\
10 / 28 / 81 \\
11 / 4 / 81 \\
11 / 12 / 81 \\
11 / 19 / 81\end{array}$ & $\begin{array}{l}254.7 \\
255.2 \\
255.1 \\
256.3 \\
255.4\end{array}$ & $\begin{array}{l}S \\
S \\
S \\
S \\
S\end{array}$ \\
\hline $\begin{array}{l}11 / 25 / 81 \\
12 / 2 / 81 \\
12 / 11 / 81 \\
12 / 16 / 81 \\
12 / 23 / 81\end{array}$ & $\begin{array}{l}255.5 \\
255.6 \\
255.8 \\
255.8 \\
255.7\end{array}$ & $\begin{array}{l}S \\
S \\
S \\
S \\
S\end{array}$ \\
\hline $\begin{array}{r}12 / 30 / 81 \\
1 / 14 / 82 \\
1 / 19 / 82 \\
1 / 22 / 82 \\
1 / 27 / 82\end{array}$ & $\begin{array}{l}255.6 \\
255.7 \\
255.6 \\
255.5 \\
255.5\end{array}$ & $\begin{array}{l}S \\
S \\
S \\
S \\
S\end{array}$ \\
\hline $\begin{array}{l}2 / 4 / 82 \\
2 / 11 / 82 \\
2 / 18 / 82 \\
2 / 25 / 82 \\
3 / 4 / 82\end{array}$ & $\begin{array}{l}255.4 \\
255.4 \\
255.5 \\
255.7 \\
255.7\end{array}$ & $\begin{array}{l}S \\
S \\
S \\
S \\
S\end{array}$ \\
\hline
\end{tabular}


Table 3. Water-level records for well $\mathrm{H}-1$, Magenta Dolomite Member of the Rustler Formation - Continued

\begin{tabular}{|c|c|c|}
\hline Date & $\begin{array}{l}\text { Water level, } \\
\text { in feet below } \\
\text { land surface }\end{array}$ & Measurement code and remarks \\
\hline $\begin{array}{l}3 / 10 / 82 \\
3 / 15 / 82 \\
3 / 25 / 82 \\
4 / 2 / 82 \\
4 / 12 / 82\end{array}$ & $\begin{array}{l}256.0 \\
256.0 \\
259.7 \\
256.7 \\
257.0\end{array}$ & $\begin{array}{l}S \\
S \\
S \\
S \\
S\end{array}$ \\
\hline $\begin{array}{l}4 / 16 / 82 \\
4 / 23 / 82 \\
4 / 27 / 82 \\
5 / 6 / 82 \\
5 / 12 / 82\end{array}$ & $\begin{array}{l}257.1 \\
257.7 \\
257.7 \\
258.0 \\
258.1\end{array}$ & $\begin{array}{l}\text { S } \\
\text { S } \\
S \\
S \\
\text { S }\end{array}$ \\
\hline $\begin{array}{l}5 / 19 / 82 \\
5 / 26 / 82 \\
6 / 4 / 82 \\
6 / 14 / 82 \\
6 / 18 / 82\end{array}$ & $\begin{array}{l}258.3 \\
258.6 \\
258.9 \\
259.2 \\
258.3\end{array}$ & $\begin{array}{l}S \\
S \\
S \\
S \\
S\end{array}$ \\
\hline $\begin{array}{l}6 / 30 / 82 \\
7 / 7 / 82 \\
7 / 16 / 82 \\
7 / 23 / 82 \\
8 / 4 / 82\end{array}$ & $\begin{array}{l}259.7 \\
259.9 \\
260.1 \\
260.4 \\
260.7\end{array}$ & $\begin{array}{l}S \\
S \\
S \\
S \\
S\end{array}$ \\
\hline $\begin{array}{l}8 / 11 / 82 \\
8 / 19 / 82 \\
8 / 26 / 82 \\
9 / 2 / 82 \\
9 / 13 / 82\end{array}$ & $\begin{array}{l}260.9 \\
261.0 \\
261.2 \\
261.3 \\
261.5\end{array}$ & $\begin{array}{l}S \\
S \\
S \\
S \\
S\end{array}$ \\
\hline $\begin{array}{r}9 / 23 / 82 \\
9 / 30 / 82 \\
10 / 8 / 82 \\
10 / 19 / 82 \\
10 / 29 / 82\end{array}$ & $\begin{array}{l}261.8 \\
261.8 \\
261.9 \\
262.2 \\
262.4\end{array}$ & $\begin{array}{l}S \\
S \\
S \\
S \\
S\end{array}$ \\
\hline
\end{tabular}


Table 3. Water-level records for well $\mathrm{H}-1$, Magenta Dolomite Member of the Rustler Formation - Concluded

\begin{tabular}{lcl}
\hline & $\begin{array}{c}\text { Water level, } \\
\text { in feet below } \\
\text { Date }\end{array}$ & \\
\hline $11 / 5 / 82$ & 262.5 & $\mathrm{~S}$ \\
$11 / 12 / 82$ & 262.5 & $\mathrm{~S}$ \\
$11 / 22 / 82$ & 262.6 & $\mathrm{~S}$ \\
$12 / 2 / 82$ & 262.6 & $\mathrm{~S}$ \\
$12 / 13 / 82$ & 262.9 & $\mathrm{~S}$ \\
& & \\
$12 / 23 / 82$ & 262.8 & $\mathrm{~S}$ \\
$12 / 29 / 82$ & 262.9 & $\mathrm{~S}$ \\
$1 / 7 / 83$ & 263.0 & $\mathrm{~S}$ \\
$1 / 19 / 83$ & 263.0 & $\mathrm{~S}$ \\
$1 / 26 / 83$ & 263.1 & $\mathrm{~S}$ \\
& & \\
$2 / 2 / 83$ & 263.1 & $\mathrm{~S}$ \\
$2 / 11 / 83$ & 263.3 & $\mathrm{~S}$ \\
$2 / 22 / 83$ & 263.4 & $\mathrm{~S}$ \\
$3 / 4 / 83$ & 263.5 & $\mathrm{~S}$ \\
$3 / 10 / 83$ & 263.7 & $\mathrm{~S}$ \\
$3 / 16 / 83$ & 263.7 & $\mathrm{~S}$ \\
$4 / 28 / 83$ & 264.1 & $\mathrm{~S}$ \\
$5 / 20 / 83$ & 264.3 & $\mathrm{~S}$ \\
$6 / 22 / 83$ & 264.5 & $\mathrm{~S}$ \\
$7 / 12 / 83$ & 264.9 & $\mathrm{~S}$ \\
$8 / 5 / 83$ & 265.1 & $\mathrm{~S}$ \\
$8 / 25 / 83$ & 265.3 & $\mathrm{~S}$ \\
$9 / 29 / 83$ & 265.5 & $\mathrm{~S}$ \\
$11 / 21 / 83$ & 266.4 & $\mathrm{~S}$ \\
$1 / 24 / 84$ & 265.8 & $\mathrm{~S}$ \\
& & \\
\hline
\end{tabular}


Table 4. Water-level records for well $\mathrm{H}-1$, Culebra Dolomite Member of the Rustler Formation

[Measurement code: A, Air-line measurement; L, Lynes Pressure Sentry System; M, M-Scope; S, Steel tape; W, Winch. Total depth is in feet below land surface. Land-surface altitude is in feet above sea level.]

Location: $22.31 \cdot 29.0623 \mathrm{FNL} .1083 \mathrm{FEL}$

Total depth: $\quad 856.0$ Land-surface altitude: $3,396.8$

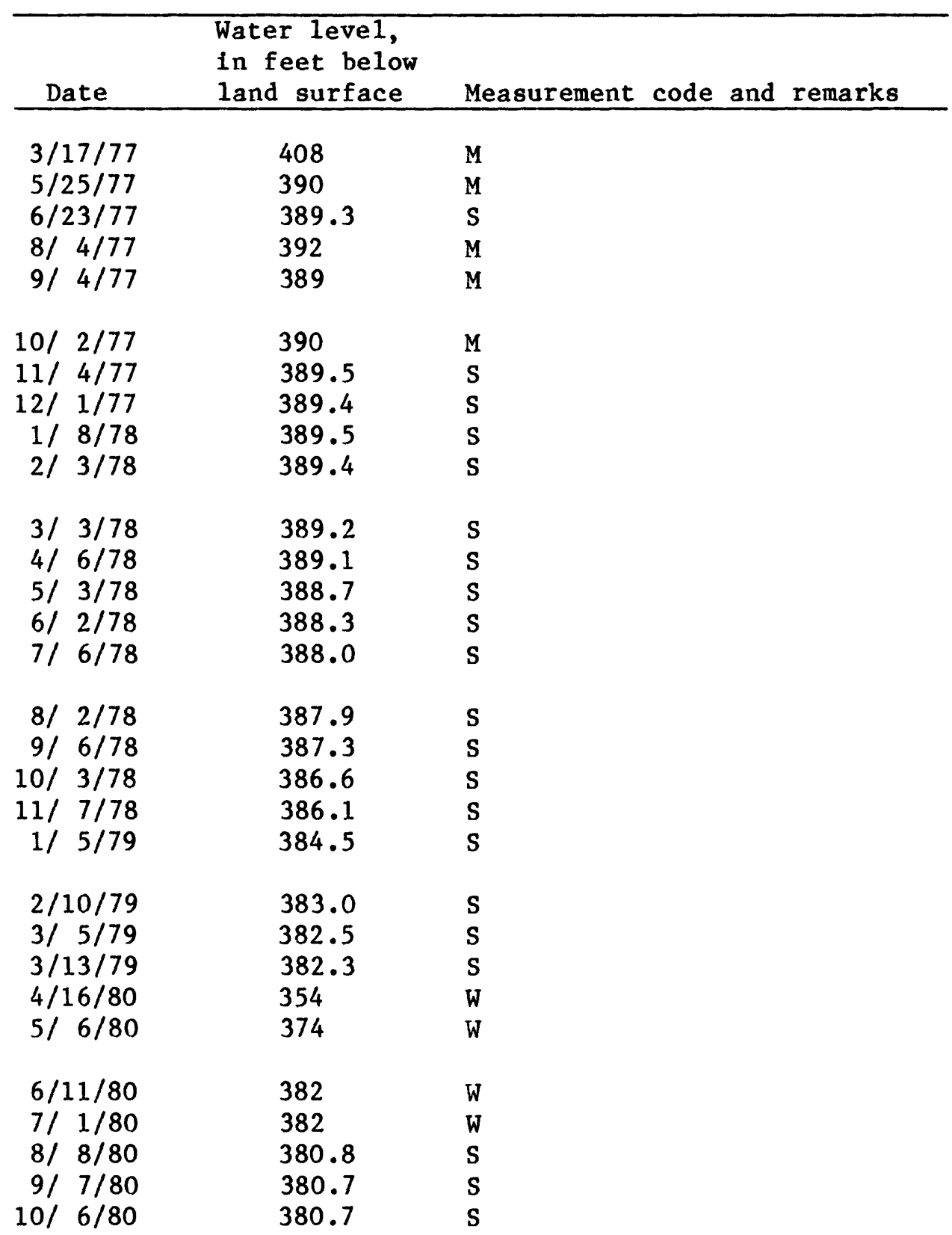


Table 4. Water-level records for well $\mathrm{H}-1$, Culebra Dolomite Member of the Rustler Formation - Continued

\begin{tabular}{lll}
\hline & $\begin{array}{c}\text { Water level, } \\
\text { in feet below } \\
\text { land surface }\end{array}$ & Measurement code and remarks \\
\hline Date & 381.2 & $\mathrm{~S}$ \\
$11 / 4 / 80$ & 381.0 & $\mathrm{~S}$ \\
$12 / 4 / 80$ & 380.8 & $\mathrm{~S}$ \\
$1 / 5 / 81$ & 380.6 & $\mathrm{~S}$ \\
$2 / 4 / 81$ & 380.4 & $\mathrm{~S}$ \\
$3 / 5 / 81$ & & \\
& 380.2 & $\mathrm{~S}$ \\
$4 / 2 / 81$ & 379.9 & $\mathrm{~S}$ \\
$5 / 4 / 81$ & 379.7 & $\mathrm{~S}$ \\
$6 / 1 / 81$ & 379.8 & $\mathrm{~S}$ \\
$7 / 6 / 81$ & 379.7 & $\mathrm{~S}$ \\
$7 / 23 / 81$ & & \\
$7 / 29 / 81$ & 379.7 & $\mathrm{~S}$ \\
$8 / 3 / 81$ & 379.7 & $\mathrm{~S}$ \\
$8 / 10 / 81$ & 379.7 & $\mathrm{~S}$ \\
$8 / 21 / 81$ & 380.5 & $\mathrm{~S}$ \\
$10 / 6 / 81$ & 405.2 & $\mathrm{~S}$ \\
$10 / 14 / 81$ & 308.5 & $\mathrm{~S}$ \\
$10 / 28 / 81$ & 413.3 & $\mathrm{~S}$ \\
$11 / 4 / 81$ & 413.6 & $\mathrm{~S}$ \\
$11 / 12 / 81$ & 409.6 & $\mathrm{~S}$ \\
$11 / 19 / 81$ & 402.2 & $\mathrm{~S}$ \\
$11 / 25 / 81$ & 394.7 & $\mathrm{~S}$ \\
$12 / 2 / 81$ & 384.8 & $\mathrm{~S}$ \\
$12 / 11 / 81$ & 372.8 & $\mathrm{~S}$ \\
$12 / 16 / 81$ & 368.3 & $\mathrm{~S}$ \\
$12 / 23 / 81$ & 365.8 & $\mathrm{~S}$ \\
$12 / 30 / 81$ & 365.8 & $\mathrm{~S}$ \\
$1 / 14 / 82$ & 368.4 & $\mathrm{~S}$ \\
$1 / 19 / 82$ & 369.5 & $\mathrm{~S}$ \\
$1 / 22 / 82$ & 370.3 & $\mathrm{~S}$ \\
$1 / 27 / 82$ & 372.0 & $\mathrm{~S}$
\end{tabular}


Table 4. Water-level records for well $\mathrm{H}-1$, Culebra Dolomite Member of the Rustler Formation - Continued

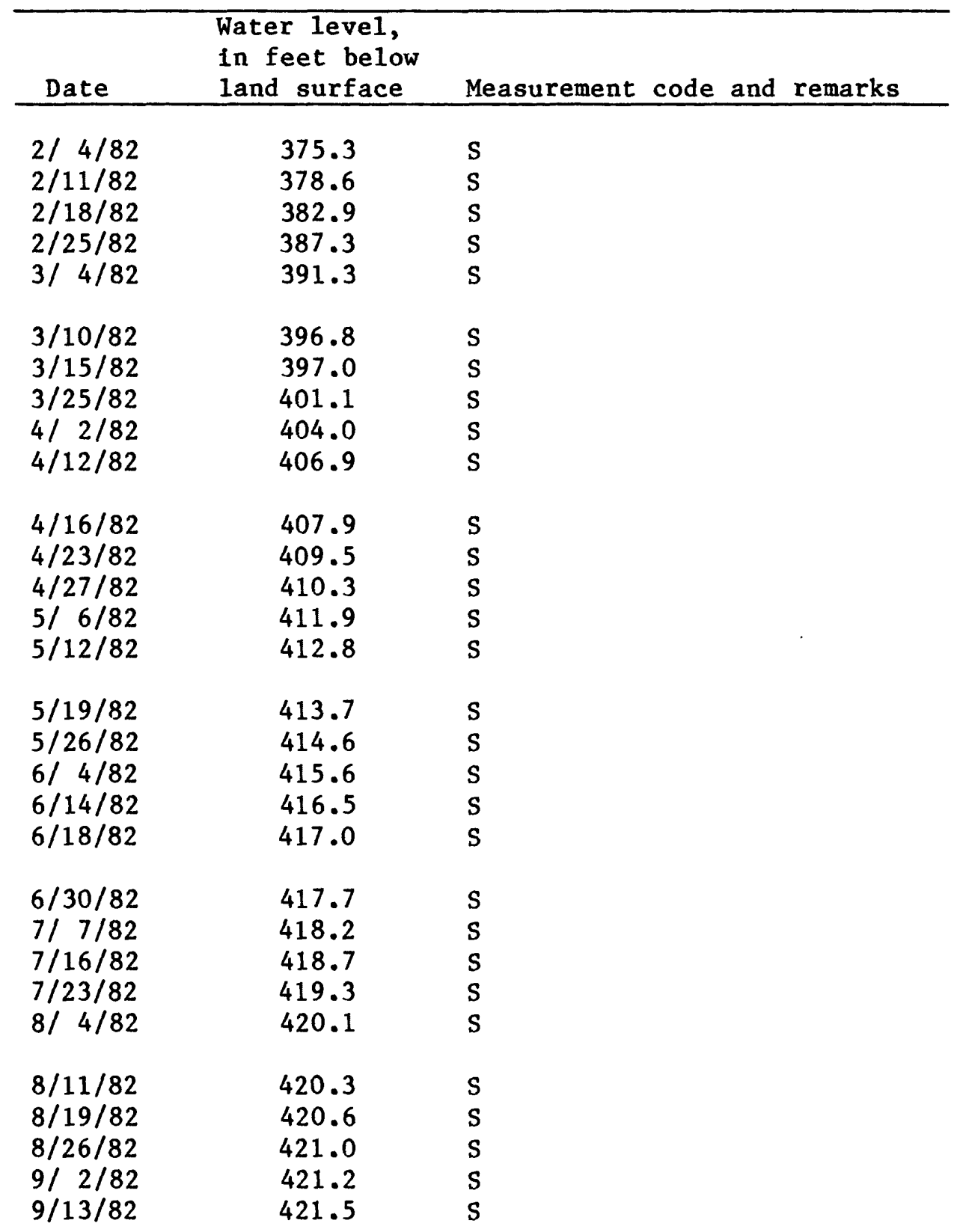


Table 4. Water-level records for well H-1, Culebra Dolomite Member of the Rustler Formation - Concluded

\begin{tabular}{ccc}
\hline & $\begin{array}{c}\text { Water level, } \\
\text { in feet below } \\
\text { 1and surface }\end{array}$ & Measurement code and remarks \\
\hline $9 / 23 / 82$ & 421.6 & $\mathrm{~S}$ \\
$9 / 30 / 82$ & 421.8 & $\mathrm{~S}$ \\
$10 / 8 / 82$ & 422.0 & $\mathrm{~S}$ \\
$10 / 19 / 82$ & 422.1 & $\mathrm{~S}$ \\
$10 / 29 / 82$ & 422.3 & $\mathrm{~S}$ \\
& & \\
$11 / 5 / 82$ & 422.4 & $\mathrm{~S}$ \\
$11 / 12 / 82$ & 422.5 & $\mathrm{~S}$ \\
$11 / 22 / 82$ & 422.6 & $\mathrm{~S}$ \\
$12 / 2 / 82$ & 422.7 & $\mathrm{~S}$ \\
$12 / 13 / 82$ & 422.9 & $\mathrm{~S}$ \\
$12 / 23 / 82$ & 423.1 & $\mathrm{~S}$ \\
$12 / 29 / 82$ & 423.2 & $\mathrm{~S}$ \\
$1 / 7 / 83$ & 423.4 & $\mathrm{~S}$ \\
$1 / 19 / 83$ & 423.3 & $\mathrm{~S}$ \\
$1 / 26 / 83$ & 423.6 & $\mathrm{~S}$ \\
& & \\
$2 / 2 / 83$ & 423.7 & $\mathrm{~S}$ \\
$2 / 11 / 83$ & 423.8 & $\mathrm{~S}$ \\
$2 / 22 / 83$ & 423.8 & $\mathrm{~S}$ \\
$3 / 4 / 83$ & 423.9 & $\mathrm{~S}$ \\
$3 / 10 / 83$ & 423.9 & $\mathrm{~S}$ \\
$3 / 16 / 83$ & 424.0 & $\mathrm{~S}$ \\
$4 / 28 / 83$ & 424.2 & $\mathrm{~S}$ \\
$5 / 20 / 83$ & 424.3 & $\mathrm{~S}$ \\
$6 / 22 / 83$ & 426.1 & $\mathrm{~S}$ \\
$7 / 12 / 83$ & 427.5 & $\mathrm{~S}$ \\
$8 / 5 / 83$ & 428.2 & $\mathrm{~S}$ \\
$8 / 25 / 83$ & 428.3 & $\mathrm{~S}$ \\
$9 / 29 / 83$ & 427.9 & $\mathrm{~S}$ \\
$11 / 21 / 83$ & 428.0 & $\mathrm{~S}$ \\
$1 / 24 / 84$ & 433.8 & $\mathrm{~S}$ \\
& & \\
\hline
\end{tabular}


Table 5. Water-level records for well $\mathrm{H}-1$, Magenta Dolomite and Culebra Dolomite Members of the Rustler Formation

[Measurement code: A, Air-line measurement; L, Lynes Pressure Sentry System; M, M-Scope; S, Steel tape; W, Winch. Total depth is in feet below land surface. Land-surface altitude is in feet above sea level.]

Location: $22 \cdot 31.29 .0623 \mathrm{FNL} .1083 \mathrm{FEL}$

Total depth: $\quad 856.0$ Land-surface altitude: $\quad 3,396.8$

\begin{tabular}{|c|c|c|}
\hline Date & $\begin{array}{l}\text { Water level, } \\
\text { in feet below } \\
\text { land surface }\end{array}$ & Measurement code and remarks \\
\hline $\begin{array}{l}2 / 28 / 80 \\
3 / 2 / 80 \\
3 / 14 / 80 \\
3 / 24 / 80\end{array}$ & $\begin{array}{l}257.1 \\
260.8 \\
257.4 \\
257.7\end{array}$ & $\begin{array}{l}S \\
S \\
S \\
S\end{array}$ \\
\hline
\end{tabular}


Table 6. Water-level records for well $\mathrm{H}-2 \mathrm{~A}$, Magenta Dolomite Member of the Rustler Formation

[Measurement code: A, Air-line measurement; L, Lynes Pressure Sentry System; M, M-Scope; S, Steel tape; W, Winch. Total depth is in feet below land surface. Land-surface altitude is in feet above sea level.]

Location: $22.31 \cdot 29.0726 \mathrm{FNL} .1698 \mathrm{FWL}$

Total depth: $\quad 563.0$ Land-surface altitude: $\quad 3,376.8$

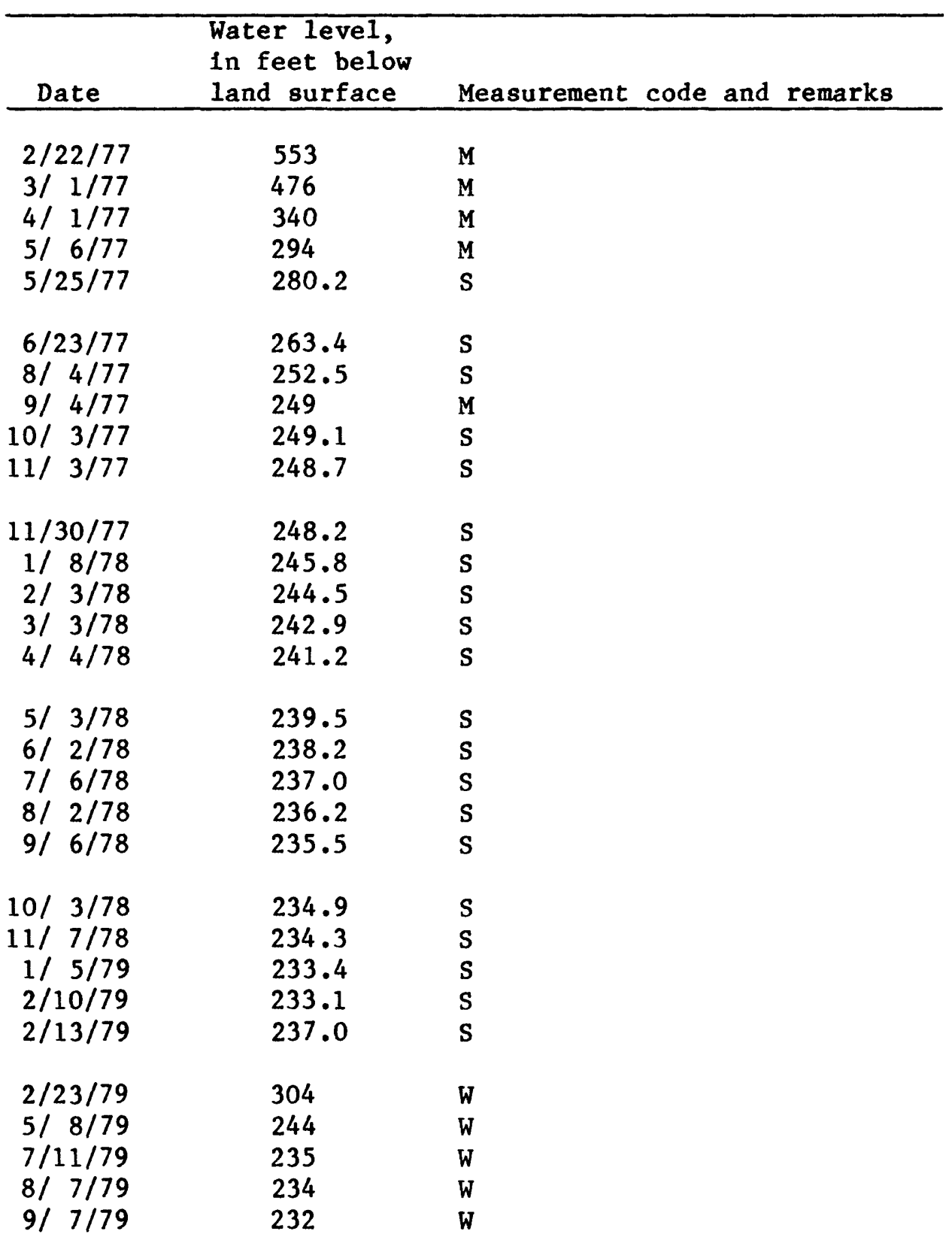


Table 6. Water-1evel records for well H-2A, Magenta Dolomite Member of the Rustler Formation - Continued

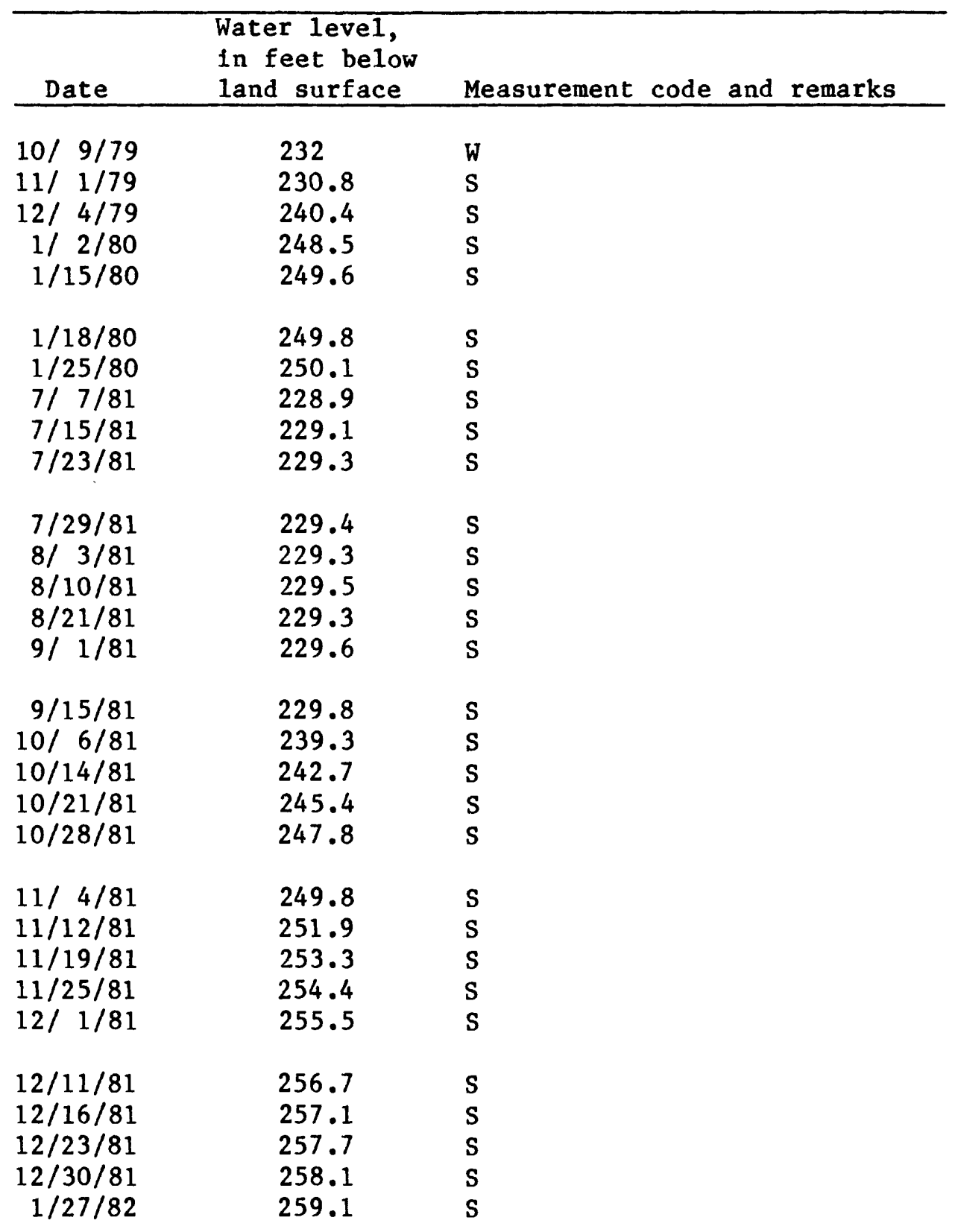


Table 6. Water-level records for well H-2A, Magenta Dolomite Member of the Rustler Formation - Continued

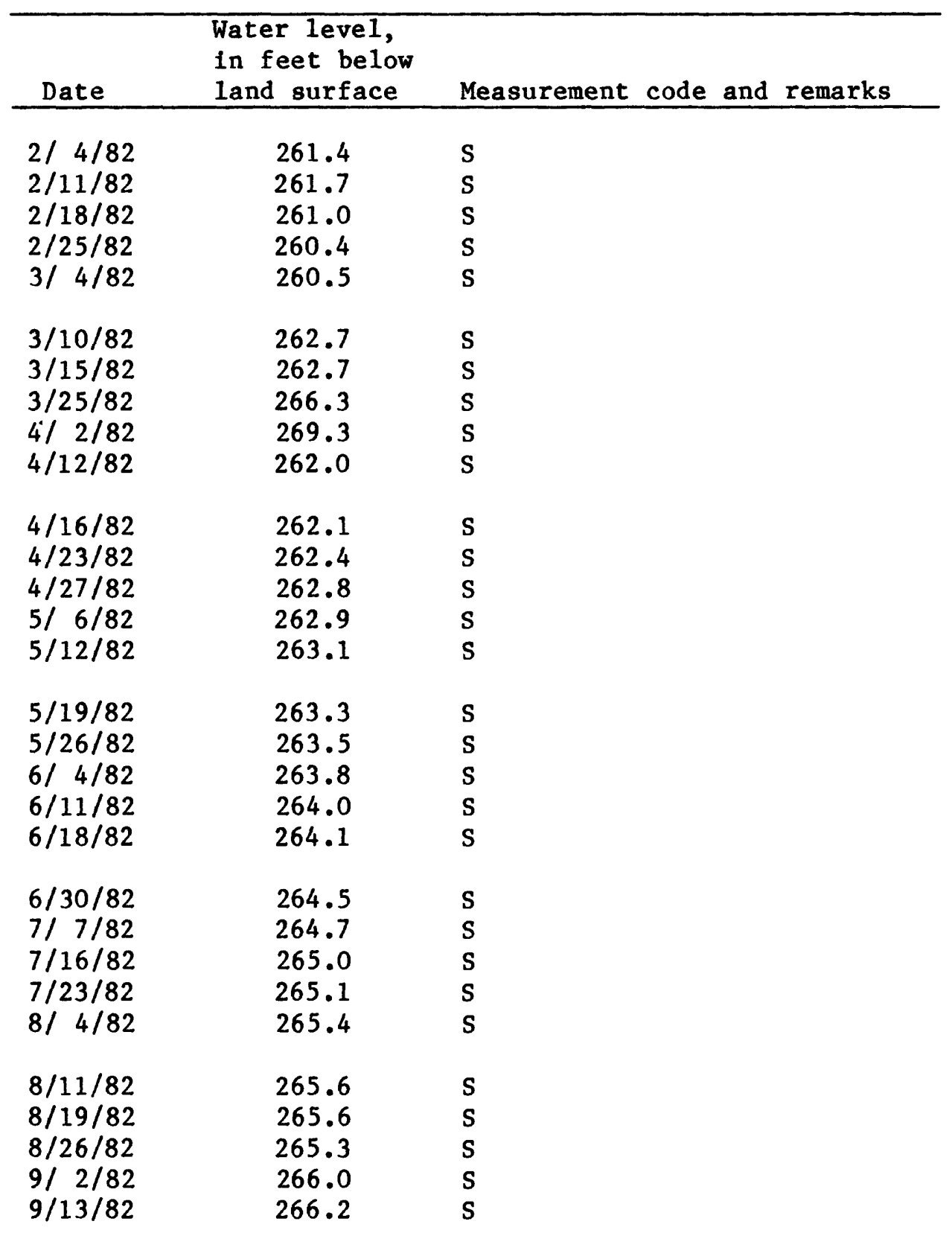


Table 6. Water-level records for well $\mathrm{H}-2 \mathrm{~A}$, Magenta Dolomite Member of the Rustler Formation - Concluded

\begin{tabular}{ccc}
\hline & $\begin{array}{l}\text { Water level, } \\
\text { 1n feet below } \\
\text { Date }\end{array}$ & \\
\hline land surface & Measurement code and remarks \\
\hline 9/23/82 & 266.4 & $\mathrm{~S}$ \\
$9 / 30 / 82$ & 266.5 & $\mathrm{~S}$ \\
$10 / 8 / 82$ & 266.5 & $\mathrm{~S}$ \\
$10 / 19 / 82$ & 266.6 & $\mathrm{~S}$ \\
$10 / 29 / 82$ & 266.9 & $\mathrm{~S}$ \\
& & \\
$11 / 5 / 82$ & 267.0 & $\mathrm{~S}$ \\
$11 / 12 / 82$ & 267.1 & $\mathrm{~S}$ \\
$11 / 22 / 82$ & 267.2 & $\mathrm{~S}$ \\
$12 / 2 / 82$ & 267.3 & $\mathrm{~S}$ \\
$12 / 13 / 82$ & 267.5 & $\mathrm{~S}$ \\
& & \\
$12 / 23 / 82$ & 267.6 & $\mathrm{~S}$ \\
$12 / 29 / 82$ & 267.7 & $\mathrm{~S}$ \\
$1 / 7 / 83$ & 267.9 & $\mathrm{~S}$ \\
$1 / 19 / 83$ & 268.0 & $\mathrm{~S}$ \\
$1 / 26 / 83$ & 268.0 & $\mathrm{~S}$ \\
& & \\
$2 / 2 / 83$ & 267.9 & $\mathrm{~S}$ \\
$2 / 11 / 83$ & 267.6 & $\mathrm{~S}$ \\
$2 / 22 / 83$ & 268.0 & $\mathrm{~S}$ \\
$3 / 4 / 83$ & 268.0 & $\mathrm{~S}$ \\
$3 / 10 / 83$ & 268.3 & $\mathrm{~S}$ \\
$3 / 16 / 83$ & 268.2 & $\mathrm{~S}$ \\
$4 / 28 / 83$ & 268.6 & $\mathrm{~S}$ \\
$5 / 20 / 83$ & 268.8 & $\mathrm{~S}$ \\
$6 / 22 / 83$ & 269.0 & $\mathrm{~S}$ \\
& & \\
\hline
\end{tabular}


Table 7. Water-level records for well H-2B, Magenta Dolomite Member of the Rustler Formation

[Measurement code: A, Afr-line measurement; L, Lynes Pressure Sentry System; M, M-Scope; S, Steel tape; W, Winch. Total depth is in feet below land surface. Land-surface altitude is in feet above sea level.]

Location: $22.31 \cdot 29.0696 \mathrm{FNL} .1661 \mathrm{FWL}$

Total depth: $\quad 661.0$ Land-surface altitude: $\quad 3,376.7$

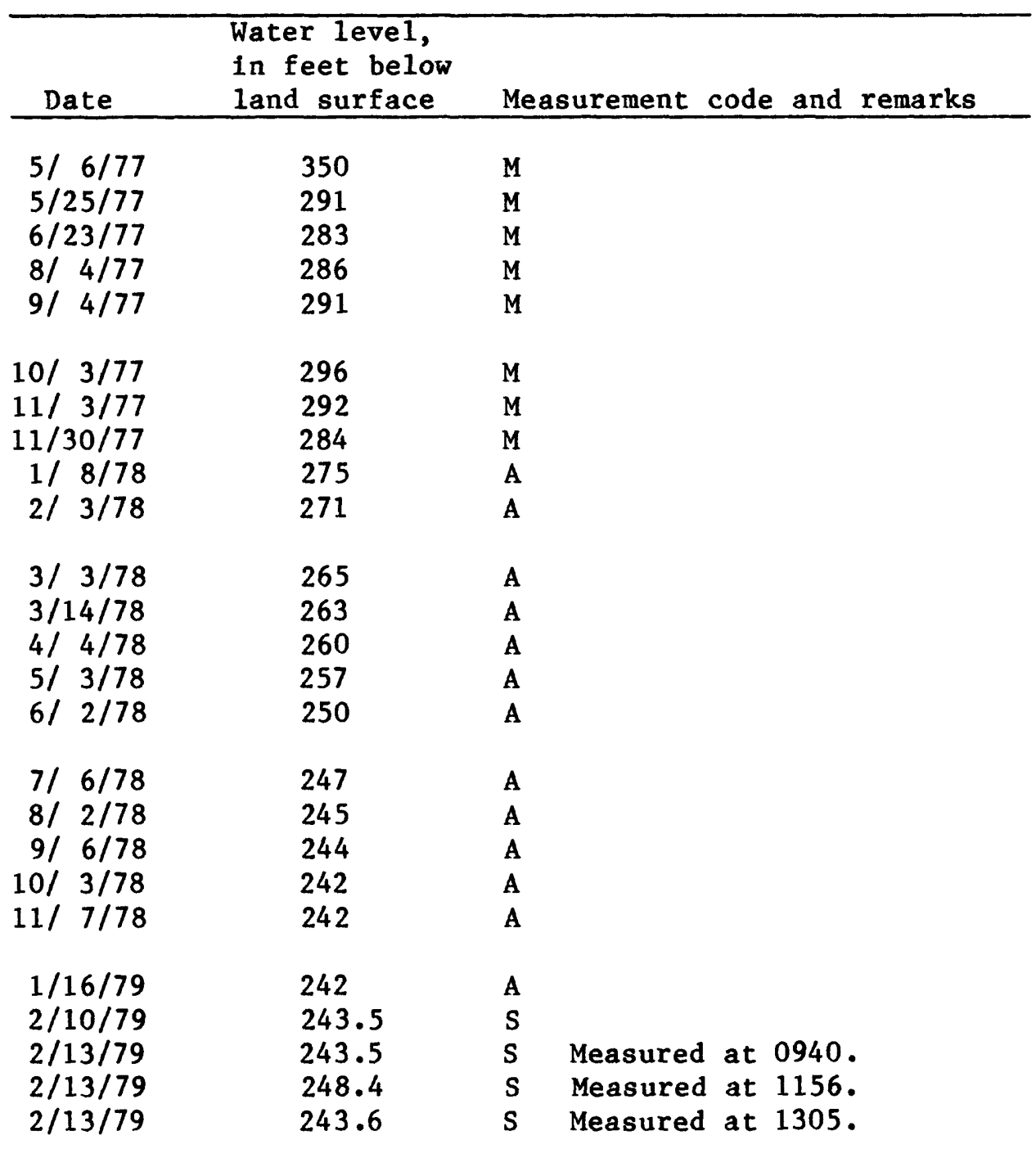


Table 7. Water-level records for well H-2B, Magenta Dolomite Member of the Rustler Formation - Concluded

\begin{tabular}{llll}
\hline & $\begin{array}{l}\text { Water level, } \\
\text { in feet below } \\
\text { land surface }\end{array}$ & Measurement code and remarks \\
\hline & & & \\
$2 / 13 / 79$ & 243.6 & S Measured at 1550. \\
$2 / 14 / 79$ & 243.9 & S Measured at 0850. \\
$2 / 14 / 79$ & 244.0 & S Measured at 1752. \\
$2 / 15 / 79$ & 244.2 & S & \\
$2 / 16 / 79$ & 244.0 & S & \\
& & & \\
$2 / 17 / 79$ & 243.3 & S & \\
$2 / 18 / 79$ & 243.1 & S & \\
\hline
\end{tabular}


Table 8. Water-level records for well H-2B, Culebra Dolomite Member of the Rustler Formation

[Measurement code: A, Air-1ine measurement; $L$, Lynes Pressure Sentry System; M, M-Scope; S, Steel tape; W, Winch. Total depth is in feet below land surface. Land-surface altitude is in feet above sea level.]

Location: $22 \cdot 31 \cdot 29.0696 \mathrm{FNL}$.1661 FWL

Total depth: $\quad 661.0$ Land-surface altitude: $\quad 3,376.7$

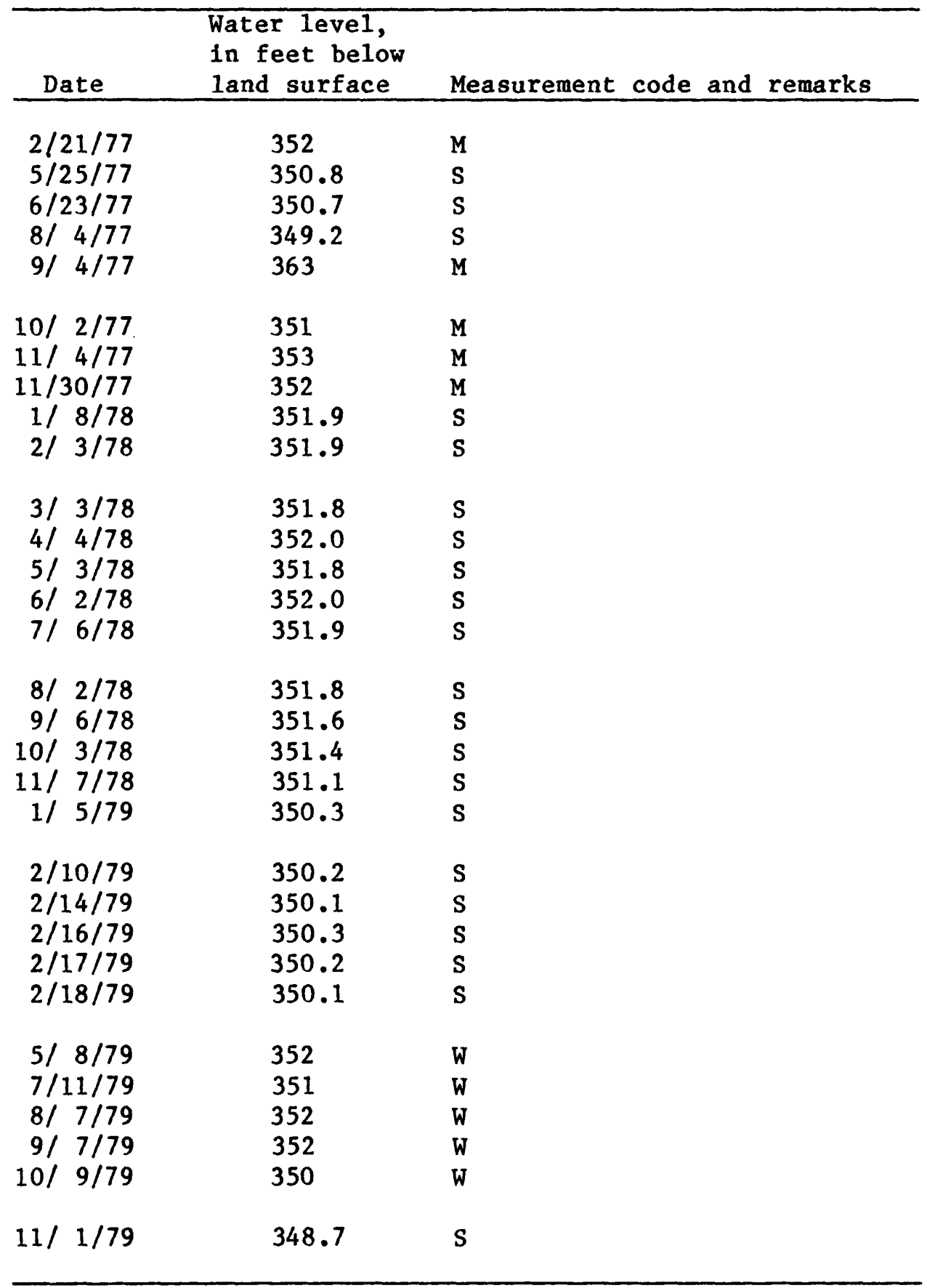


Table 9. Water-level records for well H-2B, Magenta Dolomite and Culebra Dolomite Members of the Rustler Formation

[Measurement code: A, Air-1ine measurement; L, Lynes Pressure Sentry System; M, M-Scope; S, Steel tape; W, Winch. Total depth is in feet below land surface. Land-surface altitude is in feet above sea level.]

Location: $22 \cdot 31 \cdot 29.0696 \mathrm{FNL} \cdot 1661 \mathrm{FWL}$

Total depth: $\quad 661.0$ Land-surface altitude: $\quad 3,376.7$

\begin{tabular}{lcl}
\hline & $\begin{array}{c}\text { Water level, } \\
\text { 1n feet below }\end{array}$ & \\
Date & land surface & Measurement code and remarks \\
\hline $10 / 21 / 81$ & 351.3 & $\mathrm{~S}$ \\
$10 / 28 / 81$ & 352.2 & $\mathrm{~S}$ \\
$11 / 4 / 81$ & 353.2 & $\mathrm{~S}$ \\
$11 / 12 / 81$ & 353.5 & $\mathrm{~S}$ \\
$11 / 19 / 81$ & 352.8 & $\mathrm{~S}$ \\
& & \\
$11 / 25 / 81$ & 351.8 & $\mathrm{~S}$ \\
$12 / 1 / 81$ & 350.2 & $\mathrm{~S}$ \\
$12 / 11 / 81$ & 347.7 & $\mathrm{~S}$ \\
$12 / 16 / 81$ & 346.1 & $\mathrm{~S}$ \\
$12 / 23 / 81$ & 344.2 & $\mathrm{~S}$ \\
$12 / 30 / 81$ & 343.0 & $\mathrm{~S}$ \\
$1 / 27 / 82$ & 341.8 & $\mathrm{~S}$ \\
$2 / 4 / 82$ & 343.0 & $\mathrm{~S}$ \\
$2 / 11 / 82$ & 342.3 & $\mathrm{~S}$ \\
$2 / 18 / 82$ & 342.8 & $\mathrm{~S}$ \\
& & \\
$2 / 25 / 82$ & 343.7 & $\mathrm{~S}$ \\
$3 / 4 / 82$ & 344.2 & $\mathrm{~S}$ \\
$3 / 10 / 82$ & 345.1 & $\mathrm{~S}$ \\
$3 / 15 / 82$ & 345.8 & $\mathrm{~S}$ \\
$3 / 25 / 82$ & 346.6 & $\mathrm{~S}$ \\
$4 / 2 / 82$ & 347.6 & $\mathrm{~S}$ \\
$4 / 12 / 82$ & 348.6 & $\mathrm{~S}$ \\
$4 / 16 / 82$ & 348.8 & $\mathrm{~S}$ \\
$4 / 23 / 82$ & 349.7 & $\mathrm{~S}$ \\
$4 / 27 / 82$ & 349.8 & $\mathrm{~S}$ \\
$5 / 6 / 82$ & 350.4 & $\mathrm{~S}$ \\
$5 / 12 / 82$ & 350.7 & $\mathrm{~S}$ \\
$5 / 19 / 82$ & 351.2 & $\mathrm{~S}$ \\
$5 / 26 / 82$ & 351.5 & $\mathrm{~S}$ \\
$6 / 4 / 82$ & 352.0 & $\mathrm{~S}$ \\
& & \\
& &
\end{tabular}


Table 9. Water-level records for well $\mathrm{H}-2 \mathrm{~B}$, Magenta Dolomite and Culebra Dolomite Members of the Rustler Formation

- Continued

\begin{tabular}{lll}
\hline & $\begin{array}{c}\text { Water level, } \\
\text { in feet below } \\
\text { land surface }\end{array}$ & Measurement code and remarks \\
\hline Date & 352.5 & $\mathrm{~S}$ \\
$6 / 14 / 82$ & 352.6 & $\mathrm{~S}$ \\
$6 / 18 / 82$ & 353.1 & $\mathrm{~S}$ \\
$6 / 30 / 82$ & 353.3 & $\mathrm{~S}$ \\
$7 / 7 / 82$ & 353.7 & $\mathrm{~S}$ \\
$7 / 16 / 82$ & & \\
$7 / 23 / 82$ & 354.0 & $\mathrm{~S}$ \\
$8 / 4 / 82$ & 354.4 & $\mathrm{~S}$ \\
$8 / 11 / 82$ & 354.6 & $\mathrm{~S}$ \\
$8 / 19 / 82$ & 354.8 & $\mathrm{~S}$ \\
$8 / 26 / 82$ & 355.0 & $\mathrm{~S}$ \\
$9 / 2 / 82$ & 355.1 & $\mathrm{~S}$ \\
$9 / 13 / 82$ & 355.2 & $\mathrm{~S}$ \\
$9 / 23 / 82$ & 355.5 & $\mathrm{~S}$ \\
$9 / 30 / 82$ & 355.5 & $\mathrm{~S}$ \\
$10 / 8 / 82$ & 355.6 & $\mathrm{~S}$ \\
$10 / 19 / 82$ & 355.8 & $\mathrm{~S}$ \\
$10 / 29 / 82$ & 356.1 & $\mathrm{~S}$ \\
$11 / 5 / 82$ & 356.2 & $\mathrm{~S}$ \\
$11 / 12 / 82$ & 356.2 & $\mathrm{~S}$ \\
$11 / 22 / 82$ & 356.3 & $\mathrm{~S}$ \\
$12 / 2 / 82$ & 356.5 & $\mathrm{~S}$ \\
$12 / 13 / 82$ & 357.0 & $\mathrm{~S}$ \\
$12 / 23 / 82$ & 357.1 & $\mathrm{~S}$ \\
$12 / 29 / 82$ & 357.3 & $\mathrm{~S}$ \\
$1 / 7 / 83$ & 357.4 & $\mathrm{~S}$ \\
$1 / 19 / 83$ & 357.5 & $\mathrm{~S}$ \\
$1 / 26 / 83$ & 357.4 & $\mathrm{~S}$ \\
$2 / 2 / 83$ & 357.4 & $\mathrm{~S}$ \\
$2 / 11 / 83$ & 357.4 & $\mathrm{~S}$ \\
$2 / 22 / 83$ & 357.4 & $\mathrm{~S}$ \\
& &
\end{tabular}


Table 9. Water-level records for well $\mathrm{H}-2 \mathrm{~B}$, Magenta Dolomite and Culebra Dolomite Members of the Rustler Formation

- Concluded

\begin{tabular}{|c|c|c|}
\hline Date & $\begin{array}{l}\text { Water level, } \\
\text { in feet below } \\
\text { land surface }\end{array}$ & Measurement code and remarks \\
\hline $\begin{array}{l}3 / 4 / 83 \\
3 / 10 / 83 \\
3 / 16 / 83 \\
4 / 28 / 83 \\
5 / 20 / 83\end{array}$ & $\begin{array}{l}357.3 \\
357.5 \\
357.3 \\
357.4 \\
357.4\end{array}$ & $\begin{array}{l}\text { S } \\
\text { S } \\
S \\
\text { S } \\
\text { S }\end{array}$ \\
\hline $6 / 22 / 83$ & 357.8 & S \\
\hline
\end{tabular}


Table 10. Water-level records for well H-2C, Culebra Dolomite Member of the Rustler Formation

[Measurement code: A, Air-line measurement; L, Lynes Pressure Sentry System; M, M-Scope; S, Steel tape; W, Winch. Total depth is in feet below land surface. Land-surface altitude is in feet above sea level.]

Location: $22.31 \cdot 29.0637$ FNL.1709FWL

Total depth: $\quad 795.0$ Land-surface altitude: $\quad 3,376.8$

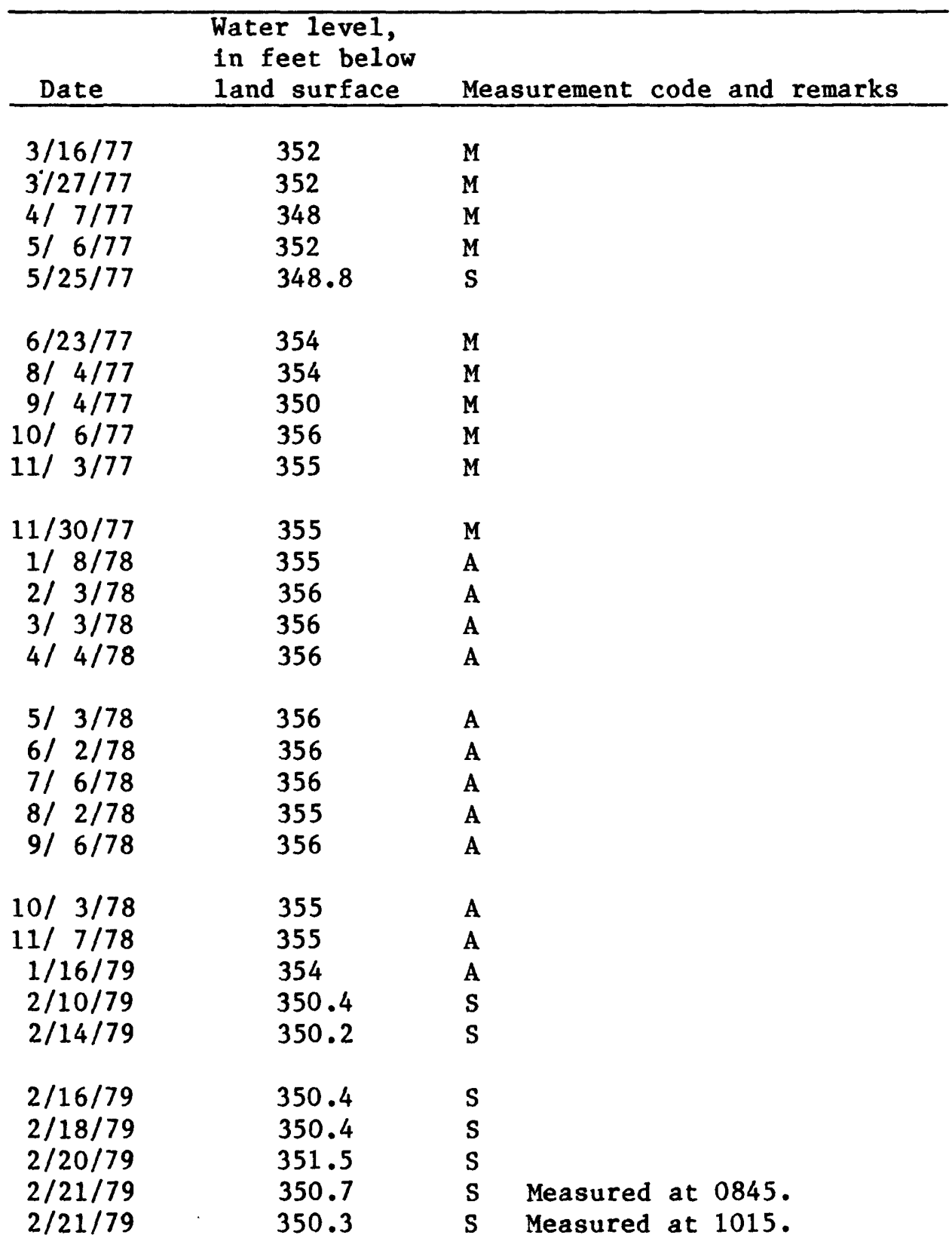


Table 10. Water-level records for well H-2C, Culebra Dolomite Member of the Rustler Formation - Continued

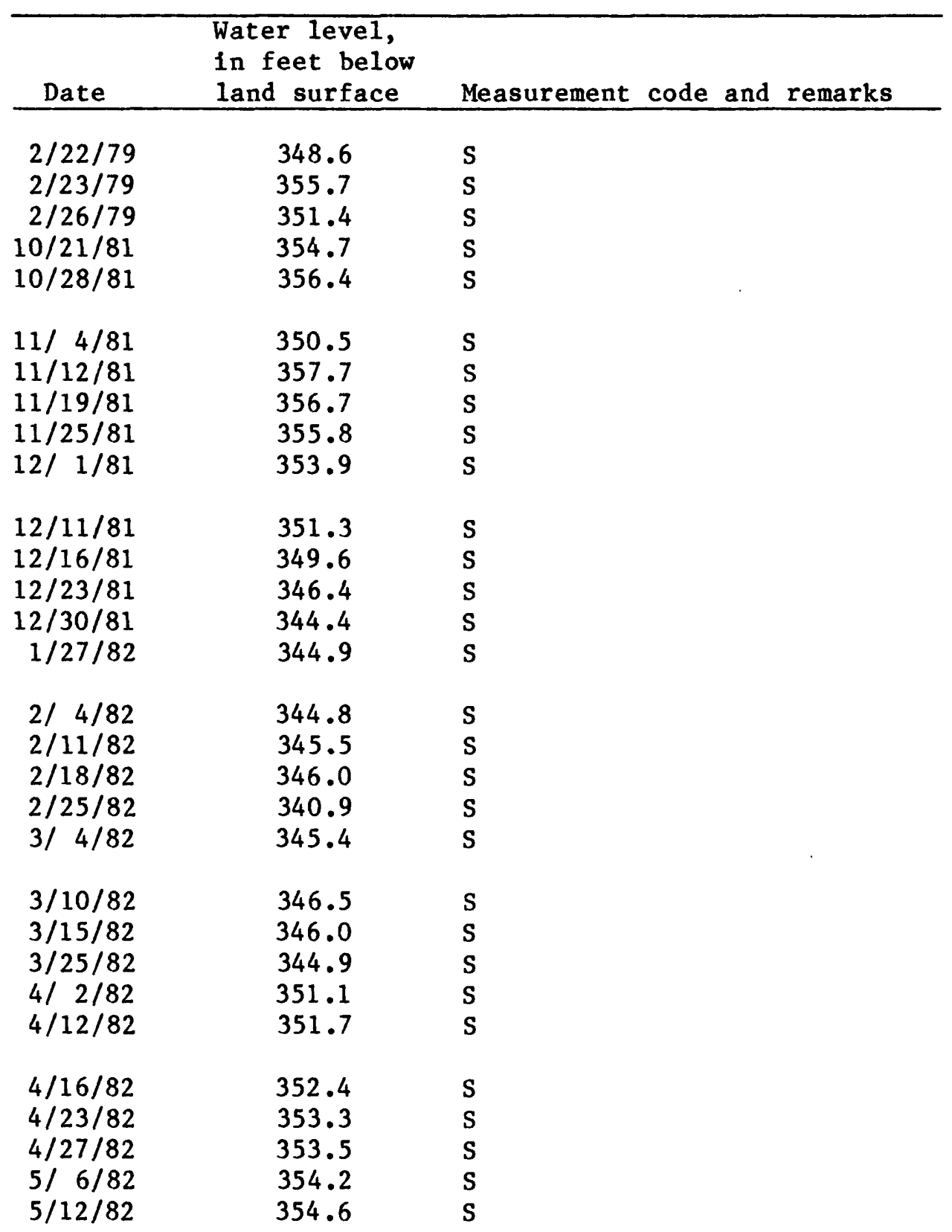


Table 10. Water-level records for well H-2C, Culebra Dolomite Member of the Rustler Formation - Continued

\begin{tabular}{|c|c|c|}
\hline Date & $\begin{array}{l}\text { Water level, } \\
\text { in feet below } \\
\text { land surface }\end{array}$ & Measurement code and remarks \\
\hline $\begin{array}{l}5 / 19 / 82 \\
5 / 26 / 82 \\
6 / 4 / 82 \\
6 / 14 / 82 \\
6 / 18 / 82\end{array}$ & $\begin{array}{l}355.0 \\
355.4 \\
355.9 \\
356.2 \\
356.5\end{array}$ & $\begin{array}{l}S \\
S \\
S \\
S \\
S\end{array}$ \\
\hline $\begin{array}{l}6 / 30 / 82 \\
7 / 7 / 82 \\
7 / 16 / 82 \\
7 / 23 / 82 \\
8 / 4 / 82\end{array}$ & $\begin{array}{l}357.2 \\
357.3 \\
357.8 \\
358.1 \\
356.9\end{array}$ & $\begin{array}{l}\text { S } \\
\text { S } \\
S \\
S \\
S\end{array}$ \\
\hline $\begin{array}{l}8 / 11 / 82 \\
8 / 19 / 82 \\
8 / 26 / 82 \\
9 / 2 / 82 \\
9 / 13 / 82\end{array}$ & $\begin{array}{l}356.8 \\
359.0 \\
359.2 \\
359.4 \\
359.5\end{array}$ & $\begin{array}{l}\text { S } \\
\text { S } \\
\text { S } \\
\text { S } \\
\text { S }\end{array}$ \\
\hline $\begin{array}{r}9 / 23 / 82 \\
9 / 30 / 82 \\
10 / 8 / 82 \\
10 / 19 / 82 \\
10 / 29 / 82\end{array}$ & $\begin{array}{l}359.8 \\
359.8 \\
360.0 \\
360.2 \\
360.4\end{array}$ & $\begin{array}{l}\mathrm{S} \\
\mathrm{S} \\
\mathrm{S} \\
\mathrm{S} \\
\mathrm{S}\end{array}$ \\
\hline $\begin{array}{l}11 / 5 / 82 \\
11 / 12 / 82 \\
11 / 22 / 82 \\
12 / 2 / 82 \\
12 / 13 / 82\end{array}$ & $\begin{array}{l}360.6 \\
360.6 \\
360.8 \\
361.0 \\
361.4\end{array}$ & $\begin{array}{l}S \\
S \\
S \\
S \\
S\end{array}$ \\
\hline $\begin{array}{r}12 / 23 / 82 \\
12 / 29 / 82 \\
1 / 7 / 83 \\
1 / 19 / 83 \\
1 / 26 / 83\end{array}$ & $\begin{array}{l}361.7 \\
361.8 \\
362.0 \\
362.2 \\
362.1\end{array}$ & $\begin{array}{l}S \\
S \\
S \\
S \\
S\end{array}$ \\
\hline
\end{tabular}


Table 10. Water-level records for well H-2C, Culebra Dolomite Member of the Rustler Formation - Concluded

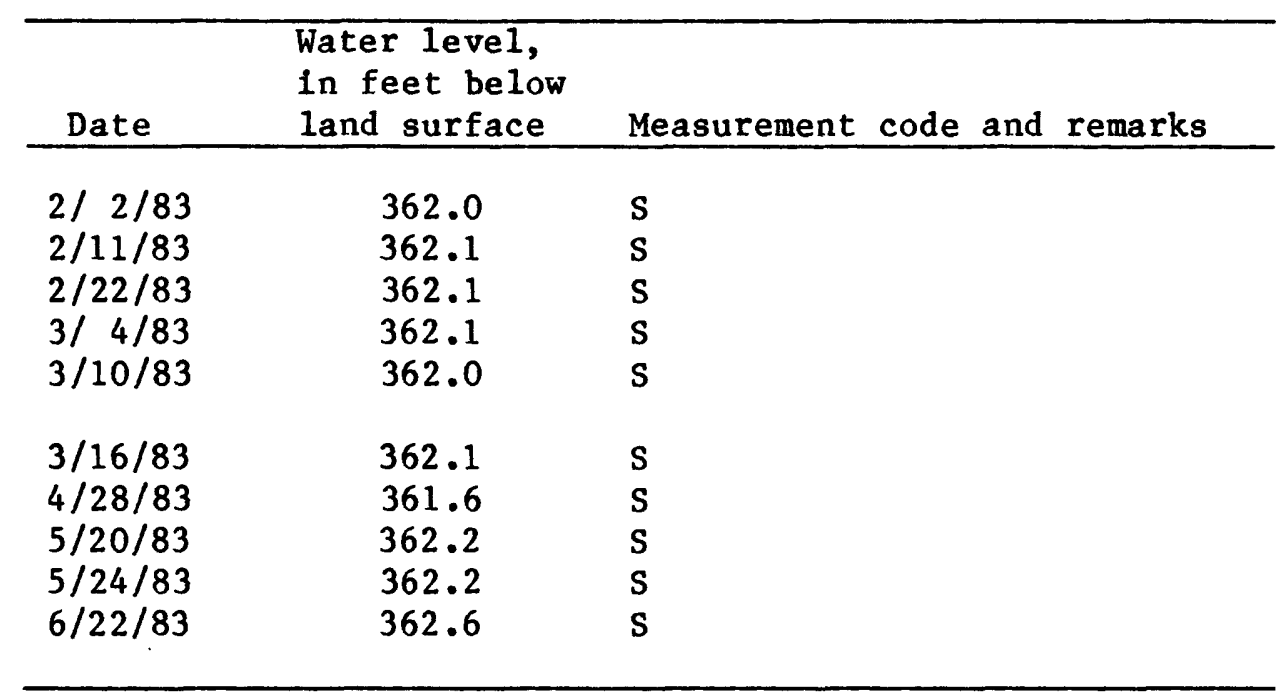


Table 11. Water-level records for well H-2C, Rustler FormationSalado Formation contact zone

[Measurement code: A, Air-line measurement; $L$, Lynes Pressure Sentry System; M, M-Scope; S, Steel tape; W, Winch. Total depth is in feet below land surface. Land-surface altitude is in feet above sea level.]

Location: $22.31 \cdot 29.0637$ FNL.1709FWL

Total depth: $\quad 795.0$ Land-surface altitude: $\quad 3,376.8$

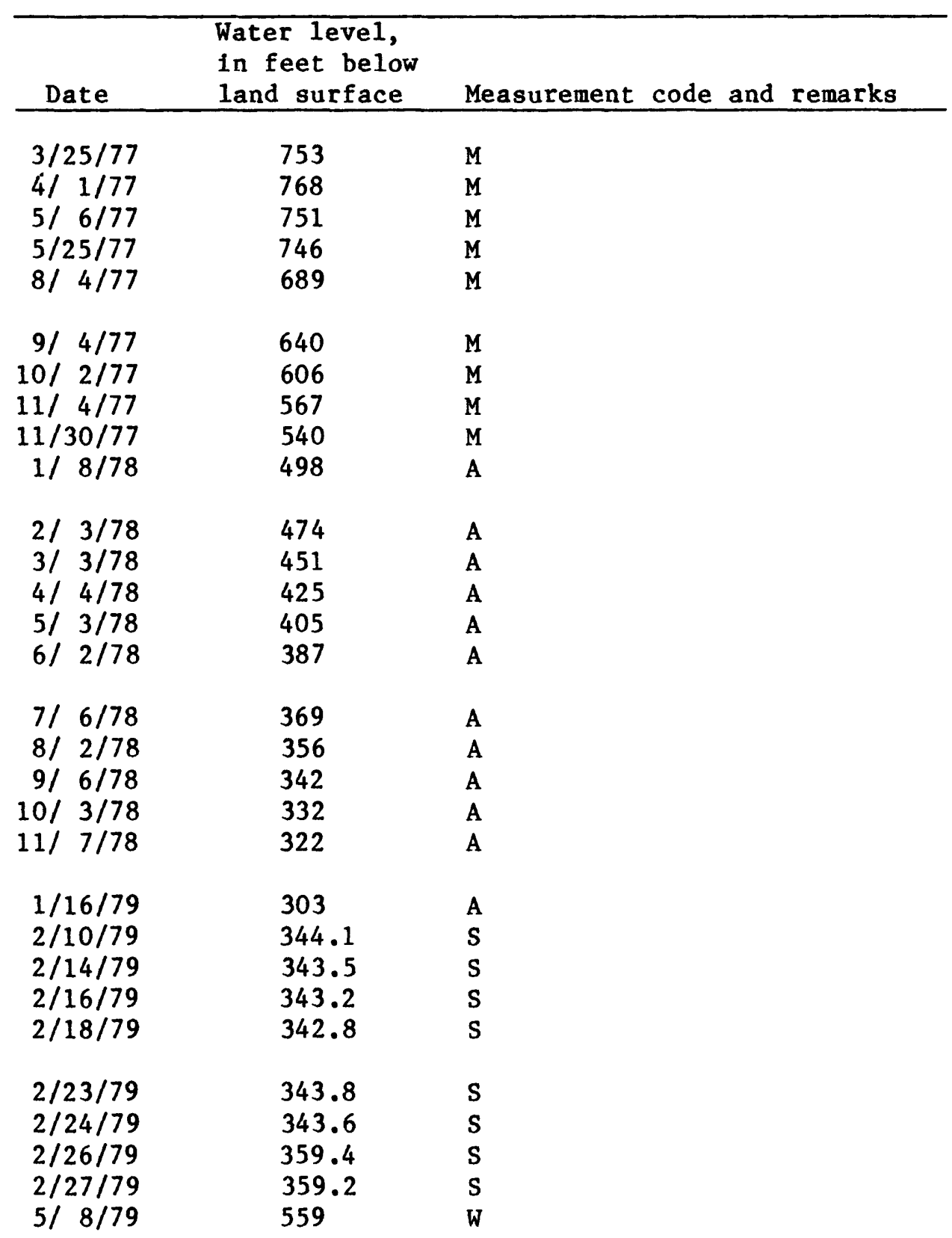


Table 11. Water-level records for well H-2C, Rustler FormationSalado Formation contact zone - Concluded

\begin{tabular}{|c|c|c|}
\hline Date & $\begin{array}{l}\text { Water level, } \\
\text { in feet below } \\
\text { land surface }\end{array}$ & Measurement code and remarks \\
\hline $\begin{array}{r}7 / 11 / 79 \\
8 / 7 / 79 \\
9 / 7 / 79 \\
10 / 9 / 79 \\
11 / 1 / 79\end{array}$ & $\begin{array}{l}484 \\
460 \\
436 \\
412 \\
400.6\end{array}$ & $\begin{array}{l}\text { W } \\
\text { W } \\
\text { W } \\
\text { W } \\
\text { S }\end{array}$ \\
\hline
\end{tabular}


Table 12. Water-level records for well $\mathrm{H}-3$, Magenta Dolomite Member of the Rustler Formation

[Measurement code: A, Air-line measurement; L, Lynes Pressure Sentry System; M, M-Scope; S, Steel tape; W, Winch. Total depth is in feet below land surface. Land-surface altitude is in feet above sea level.]

Location: $22.31 \cdot 29.2085$ FSL.0138FEL

Total depth: $\quad 894.0$ Land-surface altitude: $\quad 3,389.5$

\begin{tabular}{|c|c|c|}
\hline Date & $\begin{array}{l}\text { Water level, } \\
\text { in feet below } \\
\text { land surface }\end{array}$ & Measurement code and remarks \\
\hline $\begin{array}{r}5 / 25 / 77 \\
6 / 23 / 77 \\
8 / 4 / 77 \\
9 / 3 / 77 \\
10 / 3 / 77\end{array}$ & $\begin{array}{l}299 \\
249.7 \\
248 \\
249 \\
248\end{array}$ & $\begin{array}{l}M \\
S \\
M \\
M \\
M\end{array}$ \\
\hline $\begin{aligned} 11 / & 3 / 77 \\
12 / & 1 / 77 \\
1 / & 8 / 78 \\
2 / & 3 / 78 \\
3 / & 3 / 78\end{aligned}$ & $\begin{array}{l}245 \\
245 \\
244 \\
244 \\
245\end{array}$ & $\begin{array}{l}\text { M } \\
\text { M } \\
\text { A } \\
\text { A } \\
\text { A }\end{array}$ \\
\hline $\begin{array}{ll}4 / & 6 / 78 \\
5 / & 3 / 78 \\
6 / & 2 / 78 \\
7 / & 6 / 78 \\
8 / & 2 / 78\end{array}$ & $\begin{array}{l}242 \\
242 \\
241 \\
242 \\
240\end{array}$ & $\begin{array}{l}\text { A } \\
\text { A } \\
\text { A } \\
\text { A } \\
\text { A }\end{array}$ \\
\hline $\begin{array}{r}9 / 6 / 78 \\
10 / 3 / 78 \\
11 / 7 / 78 \\
1 / 5 / 79 \\
2 / 10 / 79\end{array}$ & $\begin{array}{l}240 \\
239 \\
238 \\
240 \\
240.7\end{array}$ & $\begin{array}{l}\text { A } \\
\text { A } \\
\text { A } \\
\text { A } \\
\text { S }\end{array}$ \\
\hline $\begin{array}{l}3 / 5 / 79 \\
4 / 6 / 79 \\
4 / 21 / 79 \\
4 / 23 / 79 \\
5 / 2 / 79\end{array}$ & $\begin{array}{l}240.5 \\
240 \\
239.5 \\
239.7 \\
239.5\end{array}$ & $\begin{array}{l}\text { S } \\
\text { A } \\
\text { S } \\
\text { S } \\
\text { S }\end{array}$ \\
\hline $\begin{array}{r}7 / 11 / 79 \\
8 / 6 / 79 \\
10 / 6 / 80 \\
11 / 4 / 80 \\
12 / 4 / 80\end{array}$ & $\begin{array}{l}240 \\
240.0 \\
259.3 \\
255.4 \\
252.8\end{array}$ & $\begin{array}{l}A \\
S \\
S \\
S \\
S\end{array}$ \\
\hline
\end{tabular}


Table 12. Water-level records for well H-3, Magenta Dolomite Member of the Rustler Formation - Continued

\begin{tabular}{|c|c|c|}
\hline Date & $\begin{array}{l}\text { Water level, } \\
\text { in feet below } \\
\text { land surface }\end{array}$ & Measurement code and remarks \\
\hline $\begin{array}{ll}1 / & 5 / 81 \\
2 / & 4 / 81 \\
3 / & 5 / 81 \\
4 / & 2 / 81 \\
5 / & 4 / 81\end{array}$ & $\begin{array}{l}251.0 \\
249.7 \\
248.4 \\
247.6 \\
246.7\end{array}$ & $\begin{array}{l}S \\
S \\
S \\
S \\
S\end{array}$ \\
\hline 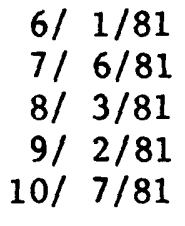 & $\begin{array}{l}246.2 \\
245.7 \\
245.3 \\
244.8 \\
244.4\end{array}$ & $\begin{array}{l}\text { S } \\
\text { S } \\
\text { S } \\
\text { S } \\
\text { S }\end{array}$ \\
\hline $\begin{array}{l}10 / 14 / 81 \\
10 / 28 / 81 \\
11 / 4 / 81 \\
11 / 12 / 81 \\
11 / 19 / 81\end{array}$ & $\begin{array}{l}244.2 \\
244.4 \\
244.1 \\
244.1 \\
243.9\end{array}$ & $\begin{array}{l}S \\
S \\
S \\
S \\
S\end{array}$ \\
\hline $\begin{array}{l}11 / 25 / 81 \\
12 / 2 / 81 \\
12 / 11 / 81 \\
12 / 16 / 81 \\
12 / 23 / 81\end{array}$ & $\begin{array}{l}243.8 \\
243.8 \\
243.8 \\
243.7 \\
243.7\end{array}$ & $\begin{array}{l}S \\
S \\
S \\
S \\
S\end{array}$ \\
\hline $\begin{array}{r}12 / 30 / 81 \\
1 / 14 / 82 \\
1 / 19 / 82 \\
1 / 22 / 82 \\
1 / 27 / 82\end{array}$ & $\begin{array}{l}243.7 \\
243.6 \\
243.5 \\
243.4 \\
243.5\end{array}$ & $\begin{array}{l}S \\
S \\
S \\
S \\
S\end{array}$ \\
\hline $\begin{array}{l}2 / 4 / 82 \\
2 / 11 / 82 \\
2 / 18 / 82 \\
2 / 25 / 82 \\
3 / 4 / 82\end{array}$ & $\begin{array}{l}243.4 \\
243.3 \\
247.2 \\
243.4 \\
243.1\end{array}$ & $\begin{array}{l}S \\
S \\
S \\
S \\
S\end{array}$ \\
\hline
\end{tabular}


Table 12. Water-level records for well H-3, Magenta Dolomite Member of the Rustler Formation - Continued

\begin{tabular}{|c|c|c|}
\hline Date & $\begin{array}{l}\text { Water level, } \\
\text { in feet below } \\
\text { land surface }\end{array}$ & Measurement code and remarks \\
\hline $\begin{array}{l}3 / 10 / 82 \\
3 / 15 / 82 \\
3 / 25 / 82 \\
4 / 2 / 82 \\
4 / 12 / 82\end{array}$ & $\begin{array}{l}245.1 \\
243.1 \\
243.1 \\
243.0 \\
243.0\end{array}$ & $\begin{array}{l}S \\
S \\
S \\
S \\
S\end{array}$ \\
\hline $\begin{array}{l}4 / 16 / 82 \\
4 / 23 / 82 \\
4 / 27 / 82 \\
5 / 6 / 82 \\
5 / 12 / 82\end{array}$ & $\begin{array}{l}242.8 \\
243.2 \\
242.9 \\
243.0 \\
242.8\end{array}$ & $\begin{array}{l}S \\
S \\
S \\
S \\
S\end{array}$ \\
\hline $\begin{array}{l}5 / 19 / 82 \\
5 / 26 / 82 \\
6 / 4 / 82 \\
6 / 11 / 82 \\
6 / 18 / 82\end{array}$ & $\begin{array}{l}241.9 \\
242.9 \\
243.0 \\
243.0 \\
242.9\end{array}$ & $\begin{array}{l}\mathrm{S} \\
\mathrm{S} \\
\mathrm{S} \\
\mathrm{S} \\
\mathrm{S}\end{array}$ \\
\hline $\begin{array}{l}6 / 30 / 82 \\
7 / 7 / 82 \\
7 / 16 / 82 \\
7 / 23 / 82 \\
8 / 4 / 82\end{array}$ & $\begin{array}{l}243.0 \\
243.0 \\
243.0 \\
243.1 \\
243.1\end{array}$ & $\begin{array}{l}S \\
S \\
S \\
S \\
S\end{array}$ \\
\hline $\begin{array}{l}8 / 11 / 82 \\
8 / 19 / 82 \\
8 / 26 / 82 \\
9 / 2 / 82 \\
9 / 13 / 82\end{array}$ & $\begin{array}{l}243.2 \\
243.1 \\
243.2 \\
243.2 \\
243.1\end{array}$ & $\begin{array}{l}S \\
S \\
S \\
S \\
S\end{array}$ \\
\hline $\begin{array}{r}9 / 23 / 82 \\
9 / 30 / 82 \\
10 / 8 / 82 \\
10 / 19 / 82 \\
10 / 29 / 82\end{array}$ & $\begin{array}{l}243.3 \\
243.2 \\
243.1 \\
243.3 \\
243.3\end{array}$ & $\begin{array}{l}S \\
S \\
S \\
S \\
S\end{array}$ \\
\hline
\end{tabular}


Table 12. Water-level records for well H-3, Magenta Dolomite Member of the Rustler Formation - Concluded

\begin{tabular}{lll}
\hline & $\begin{array}{l}\text { Water level, } \\
\text { in feet below } \\
\text { Date }\end{array}$ & \\
\hline $11 / 5 / 82$ & 243.4 & $\mathrm{~S}$ \\
$11 / 12 / 82$ & 243.4 & $\mathrm{~S}$ \\
$11 / 22 / 82$ & 243.3 & $\mathrm{~S}$ \\
$12 / 2 / 82$ & 243.1 & $\mathrm{~S}$ \\
$12 / 13 / 82$ & 243.3 & $\mathrm{~S}$ \\
$12 / 23 / 82$ & 243.2 & $\mathrm{~S}$ \\
$12 / 29 / 82$ & 243.4 & $\mathrm{~S}$ \\
$1 / 7 / 83$ & 243.3 & $\mathrm{~S}$ \\
$1 / 19 / 83$ & 243.4 & $\mathrm{~S}$ \\
$1 / 26 / 83$ & 243.3 & $\mathrm{~S}$ \\
& & \\
$2 / 2 / 83$ & 243.1 & $\mathrm{~S}$ \\
$2 / 11 / 83$ & 243.2 & $\mathrm{~S}$ \\
$2 / 22 / 83$ & 243.3 & $\mathrm{~S}$ \\
$3 / 4 / 83$ & 243.1 & $\mathrm{~S}$ \\
$3 / 10 / 83$ & 243.4 & $\mathrm{~S}$ \\
& & \\
$3 / 16 / 83$ & 243.2 & $\mathrm{~S}$ \\
$4 / 28 / 83$ & 243.4 & $\mathrm{~S}$ \\
$5 / 20 / 83$ & 243.5 & $\mathrm{~S}$ \\
$6 / 22 / 83$ & 243.7 & $\mathrm{~S}$ \\
$7 / 12 / 83$ & 243.8 & $\mathrm{~S}$ \\
$8 / 5 / 83$ & 243.9 & $\mathrm{~S}$ \\
$8 / 25 / 83$ & 243.9 & $\mathrm{~S}$ \\
$9 / 29 / 83$ & 244.0 & $\mathrm{~S}$ \\
$11 / 21 / 83$ & 240.2 & $\mathrm{~S}$ \\
\hline & & \\
\hline
\end{tabular}


Table 13. Water-level records for well H-3, Culebra Dolomite Member of the Rustler Formation

[Measurement code: A, Air-line measurement; L, Lynes Pressure Sentry System; M, M-Scope; S, Steel tape; W, Winch. Total depth is in feet below land surface. Land-surface altitude is in feet above sea level.]

Location: 22.31.29.2085FSL.0138FEL

Total depth: $\quad 894.0$ Land-surface altitude: $\quad 3,389.5$

\begin{tabular}{|c|c|c|}
\hline Date & $\begin{array}{l}\text { Water level, } \\
\text { in feet below } \\
\text { land surface }\end{array}$ & Measurement code and remarks \\
\hline $\begin{array}{l}5 / 25 / 77 \\
6 / 23 / 77 \\
8 / 4 / 77 \\
9 / 4 / 77 \\
9 / 28 / 77\end{array}$ & $\begin{array}{l}405.1 \\
405.2 \\
404.8 \\
404 \\
407\end{array}$ & $\begin{array}{l}S \\
S \\
S \\
M \\
M\end{array}$ \\
\hline $\begin{aligned} 11 / & 4 / 77 \\
12 / & 1 / 77 \\
1 / & 8 / 78 \\
2 / & 3 / 78 \\
3 / & 3 / 78\end{aligned}$ & $\begin{array}{l}404.9 \\
404.5 \\
404.6 \\
404.2 \\
403.8\end{array}$ & $\begin{array}{l}S \\
S \\
S \\
S \\
S\end{array}$ \\
\hline $\begin{array}{ll}4 / & 4 / 78 \\
5 / & 3 / 78 \\
6 / & 2 / 78 \\
7 / & 6 / 78 \\
8 / & 2 / 78\end{array}$ & $\begin{array}{l}403.5 \\
402.8 \\
402.7 \\
402.2 \\
401.8\end{array}$ & $\begin{array}{l}\mathrm{S} \\
\mathrm{S} \\
\mathrm{S} \\
\mathrm{S} \\
\mathrm{S}\end{array}$ \\
\hline $\begin{array}{r}9 / 6 / 78 \\
10 / 3 / 78 \\
11 / 7 / 78 \\
1 / 5 / 79 \\
2 / 10 / 79\end{array}$ & $\begin{array}{l}401.4 \\
401.0 \\
400.4 \\
399.0 \\
398.8\end{array}$ & $\begin{array}{l}S \\
S \\
S \\
S \\
S\end{array}$ \\
\hline $\begin{array}{rr}3 / & 5 / 79 \\
7 / 11 / 79 \\
8 / 6 / 79 \\
9 / 7 / 80 \\
10 / 6 / 80\end{array}$ & $\begin{array}{l}398.7 \\
397.7 \\
397.8 \\
390 \\
394\end{array}$ & $\begin{array}{l}\text { S } \\
\text { S } \\
\text { S } \\
W \\
W\end{array}$ \\
\hline $\begin{aligned} 11 / & 4 / 80 \\
12 / & 4 / 80 \\
1 / & 5 / 81 \\
2 / & 4 / 81 \\
3 / & 5 / 81\end{aligned}$ & $\begin{array}{l}393.3 \\
392.7 \\
392.4 \\
392.2 \\
391.9\end{array}$ & $\begin{array}{l}S \\
S \\
S \\
S \\
S\end{array}$ \\
\hline
\end{tabular}


Table 13. Water-level records for well H-3, Culebra Dolomite Member of the Rustler Formation - Continued

\begin{tabular}{lll}
\hline & $\begin{array}{c}\text { Water level, } \\
\text { in feet below } \\
\text { land surface }\end{array}$ & Measurement code and remarks \\
\hline Date & 391.8 & $\mathrm{~S}$ \\
/ $2 / 81$ & 391.5 & $\mathrm{~S}$ \\
$6 / 1 / 81$ & 391.4 & $\mathrm{~S}$ \\
$7 / 6 / 81$ & 391.5 & $\mathrm{~S}$ \\
$8 / 3 / 81$ & 391.2 & $\mathrm{~S}$ \\
& & \\
$9 / 2 / 81$ & 391.6 & $\mathrm{~S}$ \\
$10 / 7 / 81$ & 394.5 & $\mathrm{~S}$ \\
$10 / 14 / 81$ & 394.9 & $\mathrm{~S}$ \\
$10 / 28 / 81$ & 395.9 & $\mathrm{~S}$ \\
$11 / 4 / 81$ & 396.1 & $\mathrm{~S}$ \\
$11 / 12 / 81$ & 396.2 & $\mathrm{~S}$ \\
$11 / 19 / 81$ & 395.9 & $\mathrm{~S}$ \\
$11 / 25 / 81$ & 394.9 & $\mathrm{~S}$ \\
$12 / 2 / 81$ & 394.3 & $\mathrm{~S}$ \\
$12 / 11 / 81$ & 393.0 & $\mathrm{~S}$ \\
$12 / 16 / 81$ & 392.3 & $\mathrm{~S}$ \\
$12 / 23 / 81$ & 391.7 & $\mathrm{~S}$ \\
$12 / 30 / 81$ & 391.0 & $\mathrm{~S}$ \\
$1 / 14 / 82$ & 390.6 & $\mathrm{~S}$ \\
$1 / 19 / 82$ & 390.5 & $\mathrm{~S}$ \\
$1 / 22 / 82$ & 390.5 & $\mathrm{~S}$ \\
$1 / 27 / 82$ & 390.4 & $\mathrm{~S}$ \\
$2 / 4 / 82$ & 390.7 & $\mathrm{~S}$ \\
$2 / 11 / 82$ & 390.8 & $\mathrm{~S}$ \\
$2 / 18 / 82$ & 391.2 & $\mathrm{~S}$ \\
$2 / 25 / 82$ & 391.8 & $\mathrm{~S}$ \\
$3 / 4 / 82$ & 391.8 & $\mathrm{~S}$ \\
$3 / 10 / 82$ & 392.4 & $\mathrm{~S}$ \\
$3 / 15 / 82$ & 392.4 & $\mathrm{~S}$ \\
$3 / 25 / 82$ & 393.5 & $\mathrm{~S}$ \\
& &
\end{tabular}


Table 13. Water-level records for well H-3, Culebra Dolomite Member of the Rustler Formation - Continued

\begin{tabular}{|c|c|c|}
\hline Date & $\begin{array}{l}\text { Water level, } \\
\text { in feet below } \\
\text { land surface }\end{array}$ & Measurement code and remarks \\
\hline $\begin{array}{l}4 / 2 / 82 \\
4 / 12 / 82 \\
4 / 16 / 82 \\
4 / 23 / 82 \\
4 / 27 / 82\end{array}$ & $\begin{array}{l}393.5 \\
394.0 \\
394.1 \\
394.8 \\
394.7\end{array}$ & $\begin{array}{l}S \\
S \\
S \\
S \\
S\end{array}$ \\
\hline $\begin{array}{l}5 / 6 / 82 \\
5 / 12 / 82 \\
5 / 19 / 82 \\
5 / 26 / 82 \\
6 / 4 / 82\end{array}$ & $\begin{array}{l}395.2 \\
395.1 \\
395.4 \\
395.6 \\
396.1\end{array}$ & $\begin{array}{l}S \\
S \\
S \\
S \\
S\end{array}$ \\
\hline $\begin{array}{l}6 / 11 / 82 \\
6 / 18 / 82 \\
6 / 30 / 82 \\
7 / 7 / 82 \\
7 / 16 / 82\end{array}$ & $\begin{array}{l}396.1 \\
396.4 \\
396.6 \\
396.9 \\
397.1\end{array}$ & $\begin{array}{l}S \\
S \\
S \\
S \\
S\end{array}$ \\
\hline $\begin{array}{l}7 / 23 / 82 \\
8 / 4 / 82 \\
8 / 11 / 82 \\
8 / 19 / 82 \\
8 / 26 / 82\end{array}$ & $\begin{array}{l}397.3 \\
397.5 \\
397.6 \\
397.8 \\
397.7\end{array}$ & $\begin{array}{l}\mathrm{S} \\
\mathrm{S} \\
\mathrm{S} \\
\mathrm{S} \\
\mathrm{S}\end{array}$ \\
\hline $\begin{array}{r}9 / 2 / 82 \\
9 / 13 / 82 \\
9 / 23 / 82 \\
9 / 30 / 82 \\
10 / 8 / 82\end{array}$ & $\begin{array}{l}398.0 \\
398.1 \\
398.1 \\
398.2 \\
398.1\end{array}$ & $\begin{array}{l}S \\
S \\
S \\
S \\
S\end{array}$ \\
\hline $\begin{array}{l}10 / 19 / 82 \\
10 / 29 / 82 \\
11 / 5 / 82 \\
11 / 12 / 82 \\
11 / 22 / 82\end{array}$ & $\begin{array}{l}398.4 \\
398.6 \\
398.6 \\
398.9 \\
398.5\end{array}$ & $\begin{array}{l}S \\
S \\
S \\
S \\
S\end{array}$ \\
\hline
\end{tabular}


Table 13. Water-level records for wel1 H-3, Culebra Dolomite Member of the Rustler Formation - Concluded

\begin{tabular}{|c|c|c|}
\hline Date & $\begin{array}{l}\text { Water level, } \\
\text { in feet below } \\
\text { land surface }\end{array}$ & Measurement code and remarks \\
\hline $12 / 2 / 82$ & 398.5 & $S$ \\
\hline $12 / 13 / 82$ & 398.5 & $\mathrm{~s}$ \\
\hline $12 / 23 / 82$ & 398.6 & S \\
\hline $12 / 29 / 82$ & 399.0 & S \\
\hline $1 / 7 / 83$ & 398.9 & $\mathrm{~S}$ \\
\hline $1 / 26 / 83$ & 399.1 & S \\
\hline $2 / 2 / 83$ & 398.9 & $\mathrm{~S}$ \\
\hline $2 / 11 / 83$ & 399.1 & S \\
\hline $2 / 22 / 83$ & 399.1 & S \\
\hline $3 / 4 / 83$ & 398.8 & S \\
\hline $3 / 10 / 83$ & 399.2 & S \\
\hline $3 / 16 / 83$ & 399.2 & $S$ \\
\hline $4 / 28 / 83$ & 399.7 & S \\
\hline $5 / 20 / 83$ & 407.0 & S \\
\hline $5 / 24 / 83$ & 410.1 & $\mathrm{~S}$ \\
\hline $6 / 22 / 83$ & 411.0 & S \\
\hline $7 / 12 / 83$ & 408.6 & $\mathrm{~s}$ \\
\hline $8 / 5 / 83$ & 406.7 & $S$ \\
\hline $8 / 25 / 83$ & 405.5 & $S$ \\
\hline $9 / 29 / 83$ & 404.2 & $\mathrm{~S}$ \\
\hline $11 / 21 / 83$ & 402.9 & S \\
\hline
\end{tabular}


Table 14. Water-level records for well $\mathrm{H}-3$, Magenta Dolomite and Culebra Dolomite Members of the Rustler Formation

[Measurement code: A, Air-line measurement; L, Lynes Pressure Sentry System; M, M-Scope; S, Steel tape; W, Winch. Total depth is in feet below land surface. Land-surface altitude is in feet above sea level.]

Location: $22.31 \cdot 29.2085 \mathrm{FSL} .0138 \mathrm{FEL}$

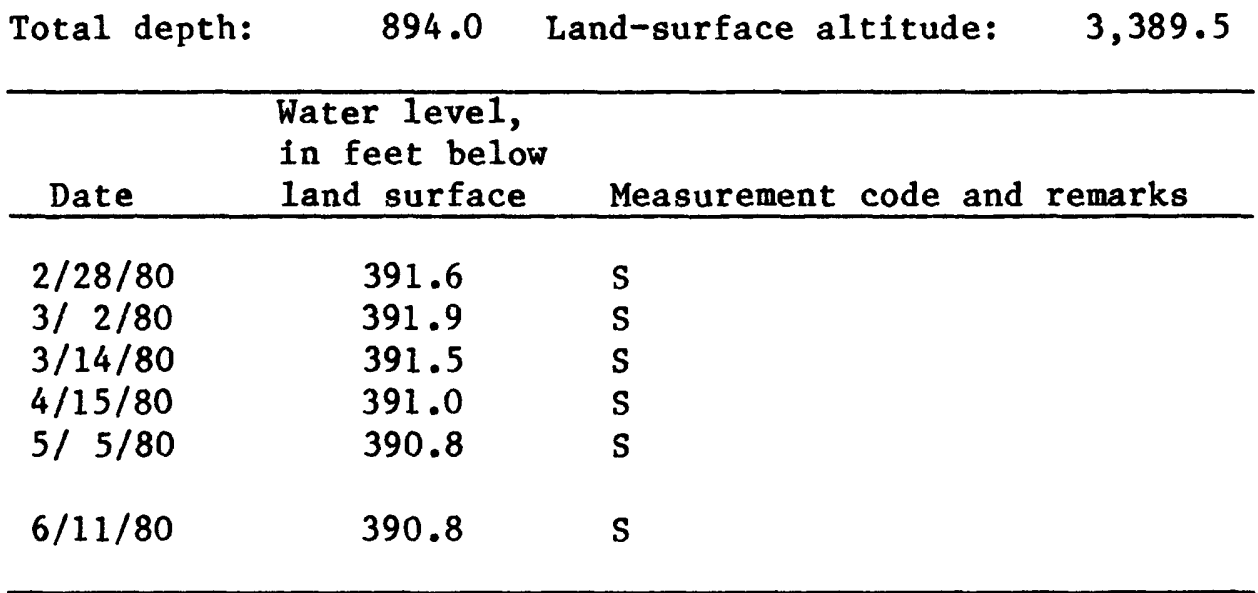


Table 15. Water-level records for well $\mathrm{H}-4 \mathrm{~A}$, Magenta Dolomite Member of the Rustler Formation

[Measurement code: A, A1r-1ine measurement; L, Lynes Pressure Sentry System; M, M-Scope; S, Steel tape; W, Winch. Total depth is in feet below land surface. Land-surface altitude is in feet above sea level.]

Location: $23.31 .05 .0546 \mathrm{FNL}$.0720FWL

Total depth: $\quad 415.0$ Land-surface altitude: $\quad 3,330.9$

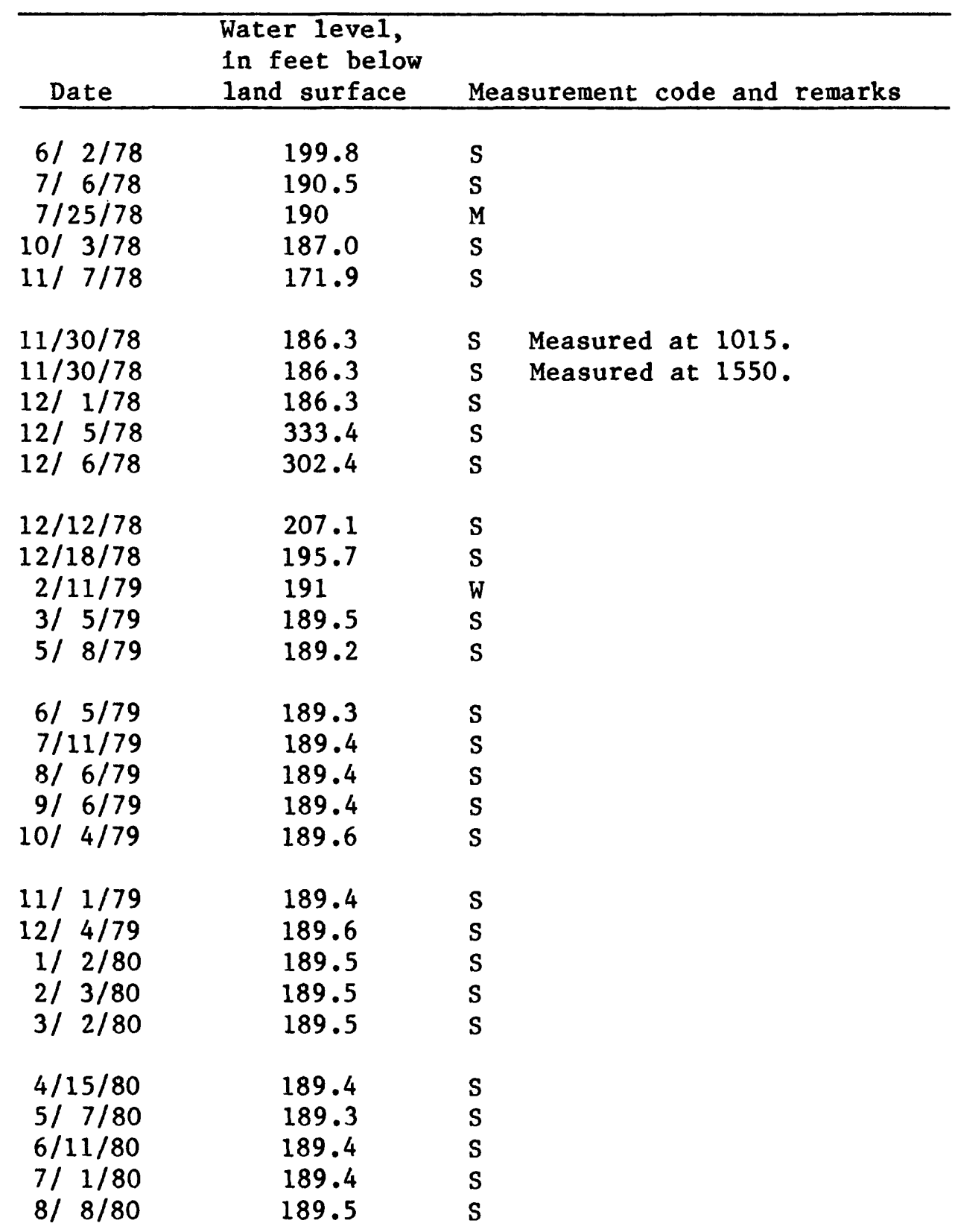


Table 15. Water-level records for well $\mathrm{H}-4 \mathrm{~A}$, Magenta Dolomite Member of the Rustler Formation - Concluded

\begin{tabular}{rcl}
\hline & $\begin{array}{l}\text { Water level, } \\
\text { in feet below } \\
\text { land surface }\end{array}$ & Measurement code and remarks \\
\hline $9 / 7 / 80$ & 189.5 & $\mathrm{~S}$ \\
$10 / 7 / 80$ & 189.1 & $\mathrm{~S}$ \\
$11 / 5 / 80$ & 189.3 & $\mathrm{~S}$ \\
$12 / 4 / 80$ & 189.3 & $\mathrm{~S}$ \\
$1 / 5 / 81$ & 189.3 & $\mathrm{~S}$ \\
\hline
\end{tabular}


Table 16. Water-level records for well H-4B, Culebra Dolomite Member of the Rustler Formation

[Measurement code: A, Air-1ine measurement; L, Lynes Pressure Sentry System; M, M-Scope; S, Steel tape; W, Winch. Total depth is in feet below land surface. Land-surface altitude is in feet above sea level.]

Location: $23.31 .05 .0498 \mathrm{FNL} .0633 \mathrm{FWL}$

Total depth: $\quad 529.0$ Land-surface altitude: $\quad 3,330.7$

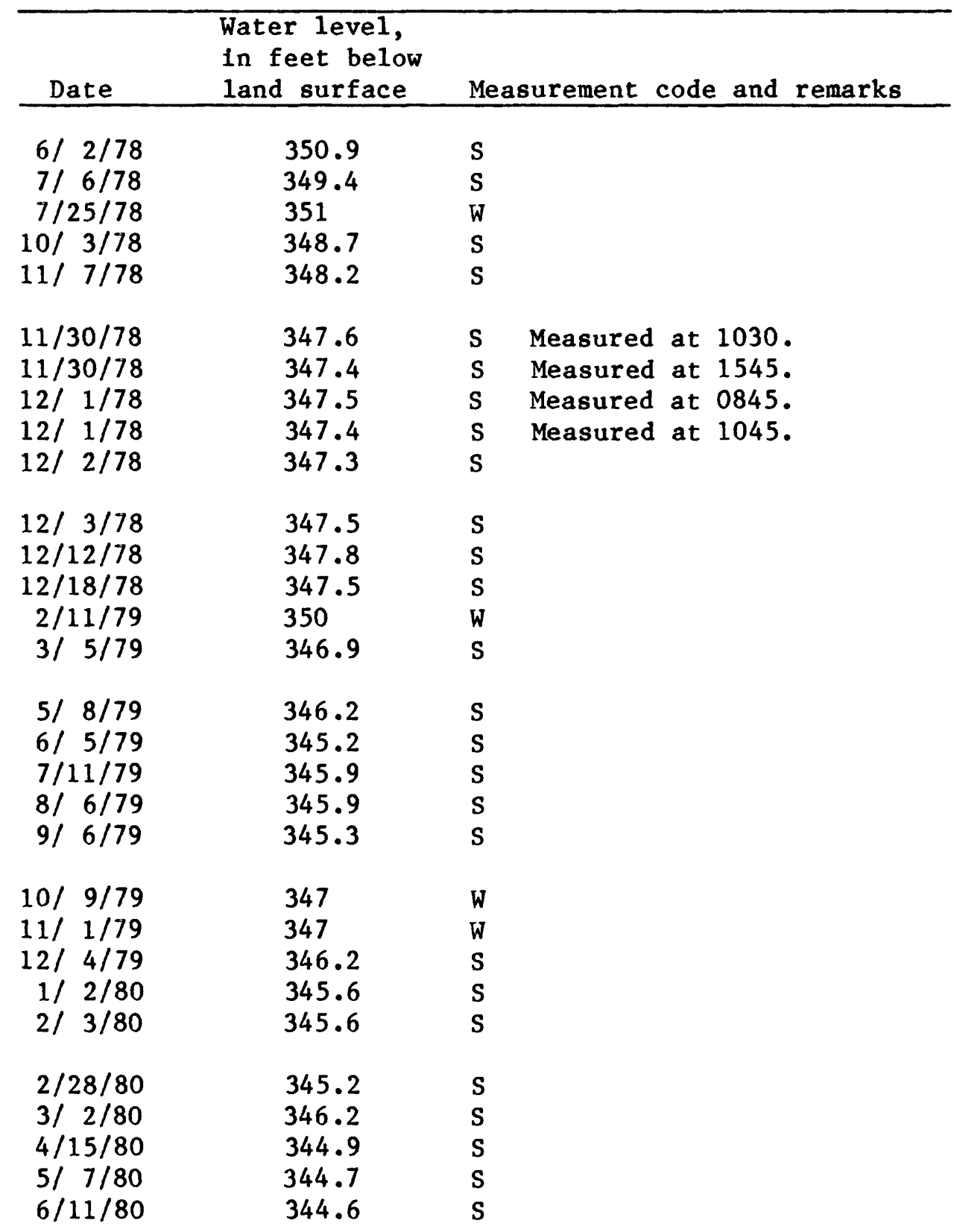


Table 16. Water-level records for well H-4B, Culebra Dolomite Member of the Rustler Formation - Concluded

\begin{tabular}{rcc}
\hline & $\begin{array}{c}\text { Water level, } \\
\text { in feet below } \\
\text { Date }\end{array}$ & \\
\hline $7 / 1 / 80$ & 344.3 & $\mathrm{~S}$ \\
$8 / 8 / 80$ & 343.9 & $\mathrm{~S}$ \\
$9 / 7 / 80$ & 343.6 & $\mathrm{~S}$ \\
$10 / 7 / 80$ & 343.3 & $\mathrm{~S}$ \\
$11 / 5 / 80$ & 342.9 & $\mathrm{~S}$ \\
& & \\
$12 / 4 / 80$ & 342.3 & $\mathrm{~S}$ \\
$1 / 5 / 81$ & 342.2 & $\mathrm{~S}$ \\
$2 / 6 / 81$ & 345.7 & $\mathrm{~S}$ \\
$3 / 5 / 81$ & 341.9 & $\mathrm{~S}$ \\
$4 / 1 / 81$ & 342.3 & $\mathrm{~S}$ \\
& & \\
$5 / 4 / 81$ & 341.7 & $\mathrm{~S}$ \\
$7 / 6 / 81$ & 341.1 & $\mathrm{~S}$ \\
$8 / 3 / 81$ & 340.5 & $\mathrm{~S}$ \\
$9 / 2 / 81$ & 339.4 & $\mathrm{~S}$ \\
$10 / 2 / 81$ & 341.9 & $\mathrm{~S}$ \\
& & \\
$11 / 4 / 81$ & 340.5 & $\mathrm{~S}$ \\
$12 / 1 / 81$ & 341 & $\mathrm{~W}$ \\
$1 / 22 / 82$ & 340.0 & $\mathrm{~S}$ \\
$2 / 18 / 82$ & 339.7 & $\mathrm{~S}$ \\
$3 / 10 / 82$ & 339.3 & $\mathrm{~S}$ \\
$4 / 15 / 82$ & 339.1 & $\mathrm{~S}$ \\
$5 / 17 / 82$ & 339.3 & $\mathrm{~S}$ \\
$6 / 22 / 82$ & 339.2 & $\mathrm{~S}$ \\
$8 / 20 / 82$ & 338.8 & $\mathrm{~S}$ \\
\hline
\end{tabular}


Table 17. Water-level records for well H-4C, Rustler FormationSalado Formation contact zone

[Measurement code: A, Afr-1ine measurement; L, Lynes Pressure Sentry System; M, M-Scope; S, Steel tape; W, Winch. Total depth is in feet below land surface. Land-surface altitude is in feet above sea level.]

Location: $23.31 .05 .0446 \mathrm{FNL} .0718 \mathrm{FWL}$

Total depth: $\quad 661.0$ Land-surface altitude: $3,333.5$

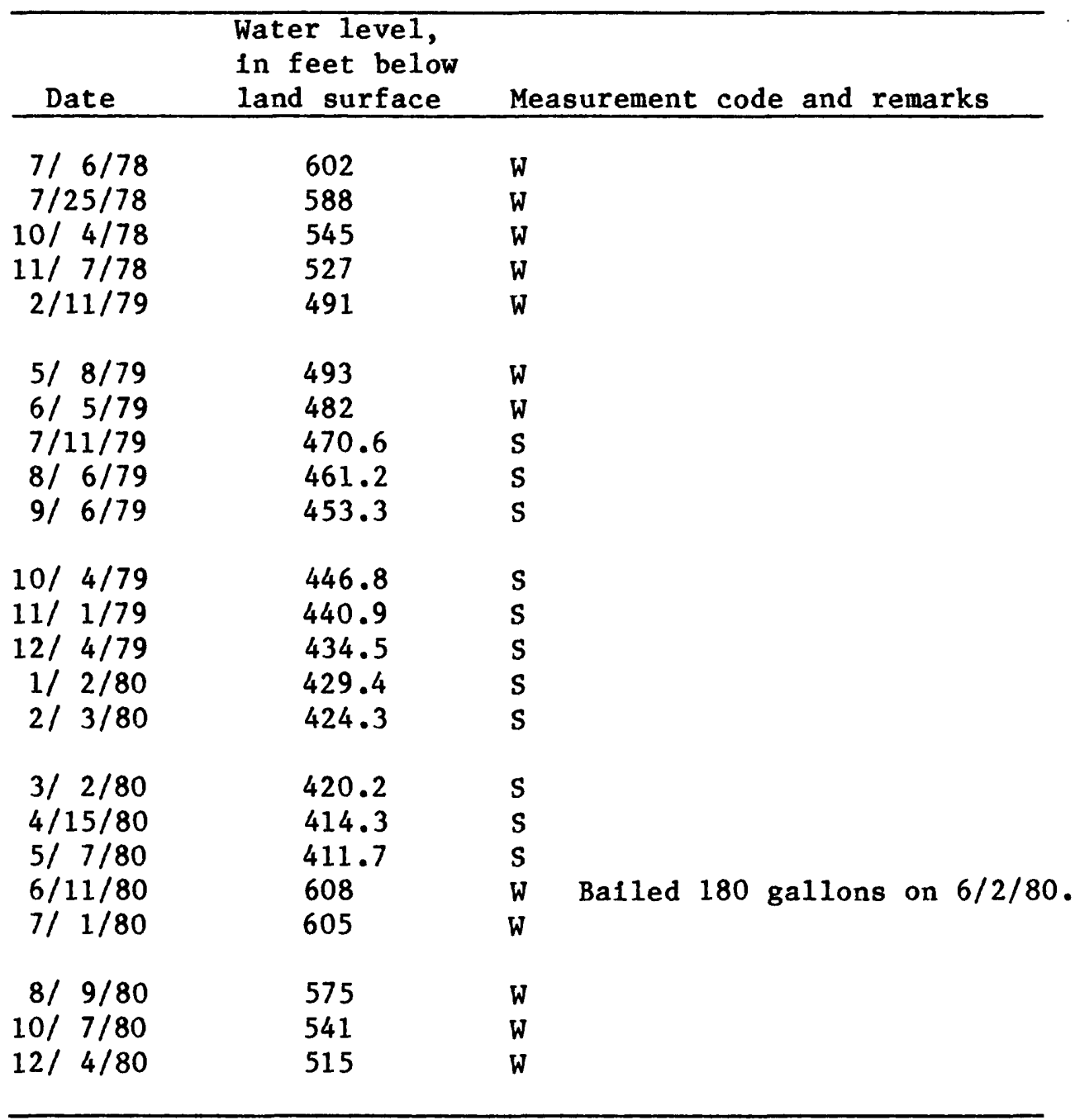


Table 18. Water-level records for well H-5A, Magenta Dolomite Member of the Rustler Formation

[Measurement code: A, Air-line measurement; L, Lynes Pressure Sentry System; M, M-Scope; S, Steel tape; W, Winch. Total depth is in feet below land surface. Land-surface altitude is in feet above sea level.]

Location: 22.31.15.1093FNL.0184FEL

Total depth: $\quad 824.0$ Land-surface altitude: $\quad 3,505.9$

\begin{tabular}{|c|c|c|}
\hline Date & $\begin{array}{l}\text { Water level, } \\
\text { in feet below } \\
\text { land surface }\end{array}$ & Measurement code and remarks \\
\hline $\begin{array}{r}7 / 7 / 78 \\
7 / 25 / 78 \\
10 / 4 / 78 \\
11 / 8 / 78 \\
12 / 2 / 78\end{array}$ & $\begin{array}{l}352 \\
348 \\
344.8 \\
345 \\
344.1\end{array}$ & $\begin{array}{l}W \\
W \\
S \\
W \\
S\end{array}$ \\
\hline $\begin{array}{r}12 / 3 / 78 \\
12 / 4 / 78 \\
12 / 6 / 78 \\
12 / 15 / 78 \\
2 / 16 / 79\end{array}$ & $\begin{array}{l}344.4 \\
344.1 \\
344.0 \\
344.1 \\
347\end{array}$ & $\begin{array}{l}\text { S } \\
\text { S } \\
\text { S } \\
\text { S } \\
\text { W }\end{array}$ \\
\hline 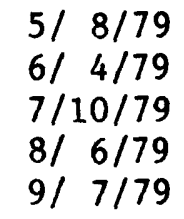 & $\begin{array}{l}344.2 \\
344.4 \\
344.4 \\
344.4 \\
344.4\end{array}$ & $\begin{array}{l}S \\
S \\
S \\
S \\
S\end{array}$ \\
\hline $\begin{aligned} 10 / & 5 / 79 \\
11 / & 1 / 79 \\
12 / & 4 / 79 \\
1 / & 2 / 80 \\
2 / & 3 / 80\end{aligned}$ & $\begin{array}{l}344.5 \\
344.0 \\
344.8 \\
344.5 \\
344.6\end{array}$ & $\begin{array}{l}S \\
S \\
S \\
S \\
S\end{array}$ \\
\hline $\begin{array}{l}3 / 2 / 80 \\
4 / 15 / 80 \\
5 / 6 / 80 \\
6 / 10 / 80 \\
7 / 1 / 80\end{array}$ & $\begin{array}{l}344.8 \\
344.4 \\
344.5 \\
344.5 \\
344.5\end{array}$ & $\begin{array}{l}\text { S } \\
\text { S } \\
S \\
S \\
\text { S }\end{array}$ \\
\hline $\begin{array}{rr}8 / & 9 / 80 \\
9 / & 8 / 80 \\
10 / & 3 / 80 \\
11 / & 5 / 80 \\
12 / & 4 / 80\end{array}$ & $\begin{array}{l}344.5 \\
344.6 \\
344.4 \\
344.6 \\
344.4\end{array}$ & $\begin{array}{l}S \\
S \\
S \\
S \\
S\end{array}$ \\
\hline $1 / 5 / 81$ & 344.5 & $S$ \\
\hline
\end{tabular}


Table 19. Water-level records for well H-5B, Culebra Dolomite Member of the Rustler Formation

[Measurement code: A, Air-1ine measurement; L, Lynes Pressure Sentry System; M, M-Scope; S, Steel tape; W, Winch. Total depth is in feet below land surface. Land-surface altitude is in feet above sea level.]

Location: 22.31.15.1007FNL.0234FEL

Total depth: $\quad 925.0$ Land-surface altitude: $\quad 3,505.7$

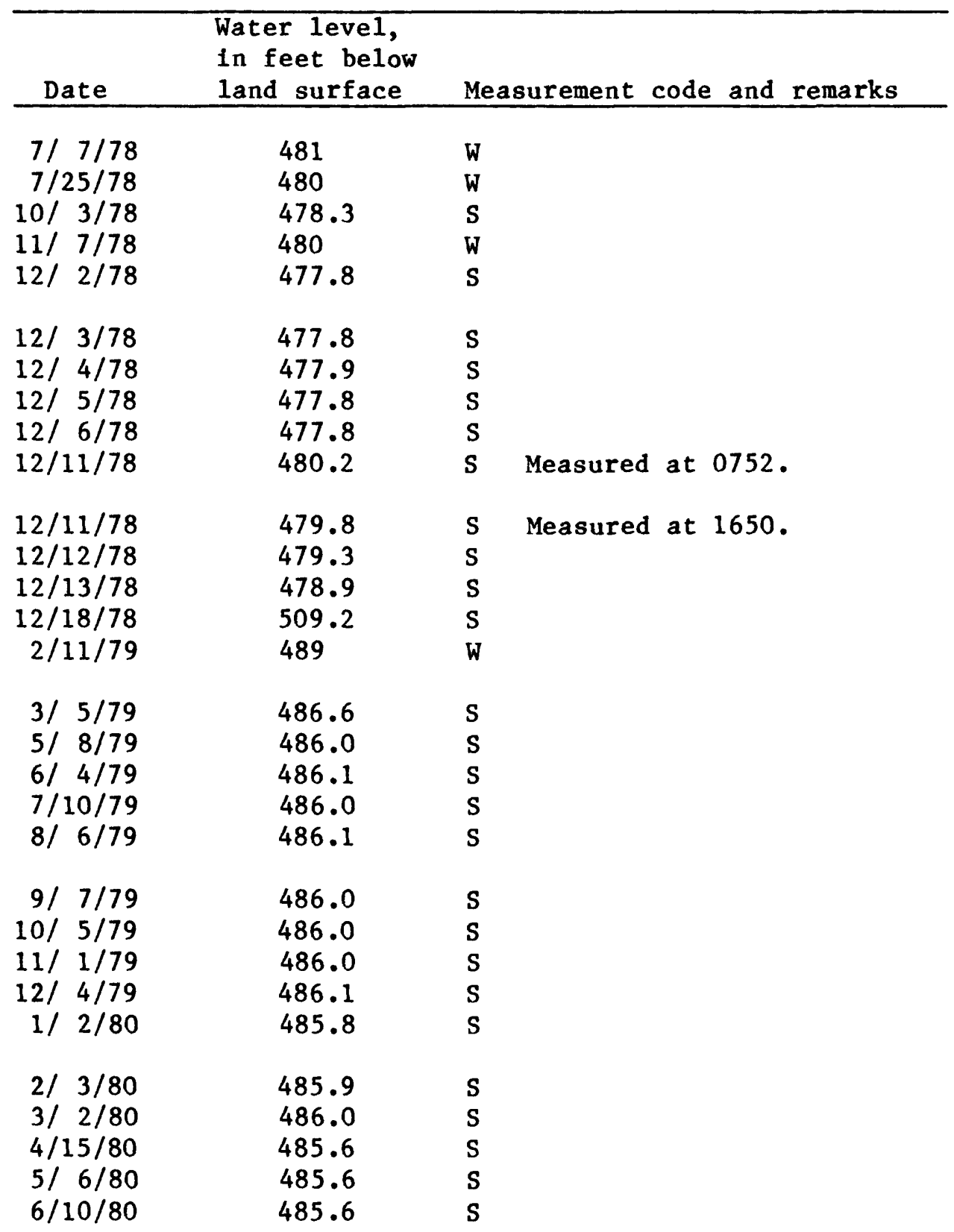


Table 19. Water-level records for well H-5B, Culebra Dolomite Member of the Rustler Formation - Concluded

\begin{tabular}{rcl}
\hline & $\begin{array}{c}\text { Water level, } \\
\text { in feet below } \\
\text { land surface }\end{array}$ & Measurement code and remarks \\
\hline $7 / 1 / 80$ & 485.6 & $\mathrm{~S}$ \\
$8 / 9 / 80$ & 485.5 & $\mathrm{~S}$ \\
$9 / 8 / 80$ & 489.5 & $\mathrm{~S}$ \\
$10 / 3 / 80$ & 485.4 & $\mathrm{~S}$ \\
$11 / 5 / 80$ & 485.4 & $\mathrm{~S}$ \\
& & \\
$12 / 4 / 80$ & 485.2 & $\mathrm{~S}$ \\
$1 / 5 / 81$ & 485.2 & $\mathrm{~S}$ \\
$3 / 4 / 81$ & 487.8 & $\mathrm{~S}$ \\
$4 / 1 / 81$ & 486.4 & $\mathrm{~S}$ \\
$5 / 4 / 81$ & 488.1 & $\mathrm{~S}$ \\
& & \\
$8 / 3 / 81$ & 489 & $\mathrm{~W}$ \\
$9 / 1 / 81$ & 488 & $\mathrm{~W}$ \\
$12 / 1 / 81$ & 492 & $\mathrm{~W}$ \\
$1 / 27 / 82$ & 488.5 & $\mathrm{~S}$ \\
$2 / 18 / 82$ & 487.7 & $\mathrm{~S}$ \\
& & \\
$3 / 10 / 82$ & 487.3 & $\mathrm{~S}$ \\
$4 / 15 / 82$ & 486.4 & $\mathrm{~S}$ \\
$5 / 17 / 82$ & 487.0 & $\mathrm{~S}$ \\
$6 / 22 / 82$ & 485.2 & $\mathrm{~S}$ \\
$8 / 20 / 82$ & 485.8 & $\mathrm{~S}$ \\
$10 / 15 / 82$ & 485.7 & $\mathrm{~S}$ \\
$12 / 13 / 82$ & 485.2 & $\mathrm{~S}$ \\
$2 / 17 / 83$ & 485.2 & $\mathrm{~S}$ \\
$10 / 18 / 84$ & 484.7 & $\mathrm{~S}$ \\
& & \\
\hline
\end{tabular}


Table 20. Water-level records for well H-5C, Rustler FormationSalado Formation contact zone

[Measurement code: A, Air-line measurement; L, Lynes Pressure Sentry System; M, M-Scope; S, Steel tape; W, Winch. Total depth is in feet below land surface. Land-surface altitude is in feet above sea level.]

Location: $22.31 \cdot 15.1006 \mathrm{FNL} .0134 \mathrm{FEL}$

Total depth: $\quad 1,076.0$ Land-surface altitude: 3,506.1

\begin{tabular}{cccl}
\hline \multicolumn{5}{c}{$\begin{array}{l}\text { Water level, } \\
\text { 1n feet below } \\
\text { 1and surface }\end{array}$} & Measurement code and remarks \\
\hline Date & -- & W & Dry. \\
$7 / 6 / 78$ & 1,062 & W & \\
$7 / 25 / 78$ & 998 & W & \\
$10 / 4 / 78$ & 547 & W & Recharge from surface-water \\
$11 / 7 / 78$ & & & runoff. \\
& & W & Do. \\
$12 / 6 / 78$ & 546 & & \\
& & W & Dry. \\
$12 / 15 / 78$ & -- & W & \\
$2 / 11 / 79$ & 1,055 & W & \\
$3 / 5 / 79$ & 1,048 & W & \\
$5 / 8 / 79$ & 1,037 & W & \\
$8 / 9 / 80$ & 1,052 & W & \\
& & W & \\
$10 / 7 / 80$ & 1,040 & W & \\
$11 / 5 / 80$ & 1,032 & & \\
$12 / 4 / 80$ & 1,026 & & \\
\hline
\end{tabular}


Table 21. Water-level records for well H-6A, Magenta Dolomite Member of the Rustler Formation

[Measurement code: A, Alr-1ine measurement; L, Lynes Pressure Sentry System; M, M-Scope; S, Steel tape; W, Winch. Total depth is in feet below land surface. Land-surface altitude is in feet above sea level.]

Location: $22 \cdot 31 \cdot 18.0284 \mathrm{FNL} .0275 \mathrm{FWL}$

Total depth: $\quad 525.0$ Land-surface altitude: $\quad 3,345.8$

\begin{tabular}{|c|c|c|}
\hline Date & $\begin{array}{l}\text { Water level, } \\
\text { in feet below } \\
\text { land surface }\end{array}$ & Measurement code and remarks \\
\hline $\begin{array}{r}7 / 25 / 78 \\
10 / 4 / 78 \\
11 / 8 / 78 \\
12 / 10 / 78 \\
12 / 11 / 78\end{array}$ & $\begin{array}{l}294 \\
292 \\
292 \\
291.5 \\
291.7\end{array}$ & $\begin{array}{l}W \\
W \\
W \\
S \\
S\end{array}$ \\
\hline $\begin{array}{r}12 / 12 / 78 \\
12 / 14 / 78 \\
2 / 16 / 79 \\
3 / 5 / 79 \\
5 / 8 / 79\end{array}$ & $\begin{array}{l}291.6 \\
292.0 \\
294 \\
292.0 \\
291.4\end{array}$ & $\begin{array}{l}S \\
S \\
W \\
S \\
S\end{array}$ \\
\hline 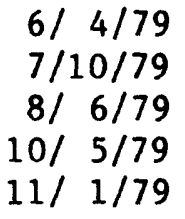 & $\begin{array}{l}291.6 \\
291.7 \\
291.4 \\
291.2 \\
291.2\end{array}$ & $\begin{array}{l}S \\
S \\
S \\
S \\
S\end{array}$ \\
\hline $\begin{array}{r}12 / 4 / 79 \\
1 / 2 / 80 \\
2 / 3 / 80 \\
3 / 2 / 80 \\
4 / 15 / 80\end{array}$ & $\begin{array}{l}291.2 \\
290.9 \\
291.0 \\
291.0 \\
290.5\end{array}$ & $\begin{array}{l}S \\
S \\
S \\
S \\
S\end{array}$ \\
\hline 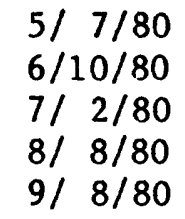 & $\begin{array}{l}290.5 \\
290.6 \\
290.6 \\
290.5 \\
290.4\end{array}$ & $\begin{array}{l}S \\
S \\
S \\
S \\
S\end{array}$ \\
\hline $\begin{array}{r}10 / 3 / 80 \\
11 / 4 / 80 \\
12 / 3 / 80 \\
1 / 5 / 81\end{array}$ & $\begin{array}{l}290.2 \\
290.1 \\
290.0 \\
289.8\end{array}$ & $\begin{array}{l}S \\
S \\
S \\
S\end{array}$ \\
\hline
\end{tabular}


Table 22. Water-level records for well H-6B, Culebra Dolomite Member of the Rustler Formation

[Measurement code: A, Alr-1ine measurement; L, Lynes Pressure Sentry System; M, M-Scope; S, Steel tape; W, Winch. Total depth is in feet below land surface. Land-surface altitude is in feet above sea leve1.]

Location: $22.31 \cdot 18.0196 \mathrm{FNL} .0322 \mathrm{FWL}$

Total depth: $\quad 640.0$ Land-surface altitude: $3,346.1$

\begin{tabular}{|c|c|c|}
\hline Date & $\begin{array}{l}\text { Water level, } \\
\text { in feet below } \\
\text { land surface }\end{array}$ & Measurement code and remarks \\
\hline $\begin{array}{r}7 / 25 / 78 \\
10 / 4 / 78 \\
11 / 8 / 78 \\
12 / 10 / 78 \\
12 / 11 / 78\end{array}$ & $\begin{array}{l}298 \\
296 \\
296 \\
294.3 \\
294.5\end{array}$ & $\begin{array}{l}W \\
W \\
W \\
S \\
S\end{array}$ \\
\hline $\begin{array}{r}12 / 12 / 78 \\
12 / 14 / 78 \\
12 / 17 / 78 \\
12 / 19 / 78 \\
2 / 16 / 79\end{array}$ & $\begin{array}{l}294.3 \\
295.3 \\
295.0 \\
294.9 \\
304\end{array}$ & $\begin{array}{l}\text { S } \\
\text { S } \\
\text { S } \\
\text { S } \\
\text { W }\end{array}$ \\
\hline $\begin{array}{ll}3 / & 5 / 79 \\
5 / & 8 / 79 \\
6 / & 4 / 79 \\
7 / 10 / 79 \\
8 / & 6 / 79\end{array}$ & $\begin{array}{l}301.4 \\
300.5 \\
300.5 \\
300.4 \\
299.9\end{array}$ & $\begin{array}{l}S \\
S \\
S \\
S \\
S\end{array}$ \\
\hline $\begin{aligned} 10 / & 9 / 79 \\
11 / & 1 / 79 \\
12 / & 4 / 79 \\
1 / & 2 / 80 \\
2 / & 3 / 80\end{aligned}$ & $\begin{array}{l}301 \\
301.0 \\
299.8 \\
299.9 \\
300.1\end{array}$ & $\begin{array}{l}\text { W } \\
\text { S } \\
\text { S } \\
\text { S } \\
\text { S }\end{array}$ \\
\hline $\begin{array}{l}2 / 28 / 80 \\
3 / 2 / 80 \\
4 / 15 / 80 \\
5 / 7 / 80 \\
6 / 10 / 80\end{array}$ & $\begin{array}{l}299.9 \\
300.4 \\
300.2 \\
300.0 \\
299.8\end{array}$ & $\begin{array}{l}S \\
S \\
S \\
S \\
S\end{array}$ \\
\hline $\begin{array}{r}7 / 2 / 80 \\
8 / 8 / 80 \\
9 / 8 / 80 \\
10 / 3 / 80 \\
11 / 4 / 80\end{array}$ & $\begin{array}{l}299.8 \\
299.7 \\
299.8 \\
299.3 \\
299.1\end{array}$ & $\begin{array}{l}\text { S } \\
\text { S } \\
\text { S } \\
\text { S } \\
\text { S }\end{array}$ \\
\hline
\end{tabular}


Table 22. Water-level records for well H-6B, Culebra Dolomite Member of the Rustler Formation - Concluded

\begin{tabular}{|c|c|c|}
\hline Date & $\begin{array}{l}\text { Water level, } \\
\text { in feet below } \\
\text { land surface }\end{array}$ & Measurement code and remarks \\
\hline $\begin{aligned} 12 / & 3 / 80 \\
1 / & 5 / 81 \\
2 / & 4 / 81 \\
3 / & 4 / 81 \\
4 / & 1 / 81\end{aligned}$ & $\begin{array}{l}298.9 \\
299.1 \\
299.5 \\
298.8 \\
299.0\end{array}$ & $\begin{array}{l}\text { S } \\
S \\
S \\
S \\
S\end{array}$ \\
\hline $\begin{aligned} 6 / & 1 / 81 \\
7 / & 6 / 81 \\
8 / & 3 / 81 \\
11 / & 4 / 81 \\
12 / & 1 / 81\end{aligned}$ & $\begin{array}{l}303.1 \\
300.4 \\
300.2 \\
300.3 \\
306.1\end{array}$ & $\begin{array}{l}S \\
S \\
S \\
S \\
S\end{array}$ \\
\hline $\begin{array}{l}1 / 27 / 82 \\
2 / 18 / 82 \\
3 / 10 / 82 \\
4 / 15 / 82 \\
5 / 18 / 82\end{array}$ & $\begin{array}{l}299.7 \\
298.8 \\
299.9 \\
299.6 \\
300.0\end{array}$ & $\begin{array}{l}\text { S } \\
S \\
S \\
S \\
S\end{array}$ \\
\hline $\begin{array}{r}6 / 22 / 82 \\
8 / 20 / 82 \\
10 / 18 / 84\end{array}$ & $\begin{array}{l}300.2 \\
300.4 \\
301.2\end{array}$ & $\begin{array}{l}S \\
S \\
S\end{array}$ \\
\hline
\end{tabular}


Table 23. Water-level records for well H-6C, Rustler FormationSalado Formation contact zone

[Measurement code: A, Air-line measurement; L, Lynes Pressure Sentry System; M, M-Scope; S, Steel tape; W, Winch. Total depth is in feet below land surface. Land-surface altitude is in feet above sea level.]

Location: $22.31 \cdot 18.0281 \mathrm{FNL} .0375 \mathrm{FWL}$

Total depth: $\quad 741.0$ Land-surface altitude: $\quad 3,346.5$

\begin{tabular}{|c|c|c|}
\hline Date & $\begin{array}{l}\text { Water level, } \\
\text { in feet below } \\
\text { land surface }\end{array}$ & Measurement code and remarks \\
\hline $\begin{array}{r}7 / 25 / 78 \\
10 / 4 / 78 \\
11 / 8 / 78 \\
2 / 11 / 79 \\
3 / 5 / 79\end{array}$ & $\begin{array}{l}624 \\
480 \\
453 \\
424 \\
418.7\end{array}$ & $\begin{array}{l}\text { W } \\
W \\
W \\
W \\
\text { S }\end{array}$ \\
\hline $\begin{array}{l}3 / 11 / 79 \\
3 / 12 / 79 \\
3 / 13 / 79 \\
3 / 18 / 79 \\
3 / 20 / 79\end{array}$ & $\begin{array}{l}417.9 \\
417.8 \\
417.8 \\
417.7 \\
417.0\end{array}$ & $\begin{array}{l}\text { S } \\
S \\
S \\
S \\
\text { S }\end{array}$ \\
\hline $\begin{array}{r}5 / 9 / 79 \\
7 / 10 / 79 \\
8 / 7 / 79 \\
10 / 5 / 79 \\
11 / 1 / 79\end{array}$ & $\begin{array}{l}540 \\
520 \\
483 \\
439.6 \\
431.8\end{array}$ & $\begin{array}{l}\text { W } \\
\text { W } \\
\text { W } \\
\text { S } \\
\text { S }\end{array}$ \\
\hline $\begin{array}{rr}12 / & 4 / 79 \\
1 / & 2 / 80 \\
2 / & 3 / 80 \\
3 / & 2 / 80 \\
4 / 15 / 80\end{array}$ & $\begin{array}{l}424.3 \\
420.1 \\
417.0 \\
415.2 \\
413.4\end{array}$ & $\begin{array}{l}S \\
S \\
S \\
S \\
S\end{array}$ \\
\hline $\begin{array}{ll}5 / & 7 / 80 \\
6 / 10 / 80 \\
7 / 2 / 80 \\
8 / & 8 / 80 \\
9 / & 8 / 80\end{array}$ & $\begin{array}{l}412.8 \\
412.1 \\
411.8 \\
411.3 \\
411.2\end{array}$ & $\begin{array}{l}S \\
S \\
S \\
S \\
S\end{array}$ \\
\hline $\begin{array}{cc}10 / & 3 / 80 \\
11 / & 4 / 80 \\
12 / & 3 / 80 \\
1 / & 5 / 81\end{array}$ & $\begin{array}{l}411.1 \\
410.8 \\
410.5 \\
412.5\end{array}$ & $\begin{array}{l}S \\
S \\
S \\
S\end{array}$ \\
\hline
\end{tabular}


Table 24. Water-level records for well H-7A, Magenta Dolomite Member of the Rustler Formation

[Measurement code: A, Air-1ine measurement; L, Lynes Pressure Sentry System; M, M-Scope; S, Steel tape; W, Winch. Total depth is in feet below land surface. Land-surface altitude is in feet above sea leve1.]

Location: $23 \cdot 30 \cdot 14 \cdot 2495 \mathrm{FNL} .2492 \mathrm{FWL}$

Total depth: $\quad 154.0$ Land-surface altitude: $\quad 3,163.6$

\begin{tabular}{|c|c|c|c|c|}
\hline Date & $\begin{array}{l}\text { Water level, } \\
\text { in feet below } \\
\text { land surface }\end{array}$ & Measurement & code and & remarks \\
\hline $\begin{array}{r}9 / 26 / 79 \\
10 / 10 / 79 \\
11 / 1 / 79 \\
12 / 4 / 79 \\
11 / 4 / 80\end{array}$ & $\begin{array}{l}154 \\
154 \\
154.3 \\
154.1 \\
-\infty\end{array}$ & $\begin{array}{ll}\text { W } & \text { Magenta } \\
\text { W } & \text { Do. } \\
\text { S } & \text { Do. } \\
\text { S } & \text { Do. } \\
& \text { Do. }\end{array}$ & dry. & \\
\hline $\begin{array}{r}6 / 2 / 81 \\
10 / 2 / 81 \\
1 / 22 / 82\end{array}$ & $\begin{array}{l}-- \\
--\end{array}$ & $\begin{array}{l}\text { Do. } \\
\text { Do. } \\
\text { Do. }\end{array}$ & & \\
\hline
\end{tabular}


Table 25. Water-level records for well H-7B, Culebra Dolomite Member of the Rustler Formation

[Measurement code: A, Air-line measurement; L, Lynes Pressure Sentry System; M, M-Scope; S, Steel tape; W, Winch. Total depth is in feet below land surface. Land-surface altitude is in feet above sea level.]

Location: $23 \cdot 30 \cdot 14 \cdot 2566 \mathrm{FNL} .2563 \mathrm{FWL}$

Total depth: $\quad 286.0$ Land-surface altitude: $\quad 3,162.6$

\begin{tabular}{|c|c|c|}
\hline Date & $\begin{array}{l}\text { Water level, } \\
\text { in feet below } \\
\text { land surface }\end{array}$ & Measurement code and remarks \\
\hline $9 / 19 / 79$ & 171 & W \\
\hline $9 / 26 / 79$ & 171 & W \\
\hline $10 / 10 / 79$ & 171 & W \\
\hline $11 / 1 / 79$ & 170.9 & $\mathbf{S}$ \\
\hline $12 / 4 / 79$ & 170.9 & S \\
\hline $1 / 2 / 80$ & 171.5 & $\mathrm{~s}$ \\
\hline $2 / 3 / 80$ & 171.6 & $\mathbf{S}$ \\
\hline $3 / 2 / 80$ & 171.8 & S \\
\hline $3 / 20 / 80$ & 171.5 & S \\
\hline $4 / 15 / 80$ & 171.3 & S \\
\hline $5 / 6 / 80$ & 171.4 & $\mathrm{~S}$ \\
\hline $6 / 10 / 80$ & 171.4 & $\mathbf{S}$ \\
\hline $7 / 1 / 80$ & 171 & W \\
\hline $8 / 2 / 80$ & 171.3 & $\mathbf{S}$ \\
\hline $9 / 8 / 80$ & 171.4 & $\mathbf{S}$ \\
\hline $10 / 8 / 80$ & 171.3 & S \\
\hline $11 / 4 / 80$ & 171.1 & $\mathbf{S}$ \\
\hline $12 / 2 / 80$ & 170.7 & $S$ \\
\hline $2 / 4 / 81$ & 170.4 & $\mathbf{S}$ \\
\hline $5 / 4 / 81$ & 170.0 & $\mathbf{S}$ \\
\hline $6 / 2 / 81$ & 170.1 & $\mathbf{S}$ \\
\hline $7 / 6 / 81$ & 170.2 & $\mathbf{S}$ \\
\hline $7 / 15 / 81$ & 170.2 & $\mathbf{S}$ \\
\hline $7 / 23 / 81$ & 170.2 & $\mathbf{S}$ \\
\hline $7 / 29 / 81$ & 170.3 & $S$ \\
\hline $8 / 4 / 81$ & 170.2 & $\mathbf{S}$ \\
\hline $8 / 10 / 81$ & 170.4 & $\mathbf{S}$ \\
\hline $8 / 21 / 81$ & 170.3 & $\mathbf{S}$ \\
\hline $9 / 1 / 81$ & 170.2 & $\mathbf{S}$ \\
\hline $9 / 14 / 81$ & 170.2 & Measured at 0920. \\
\hline
\end{tabular}


Table 25. Water-level records for well H-7B, Culebra Dolomite Member of the Rustler Formation - Concluded

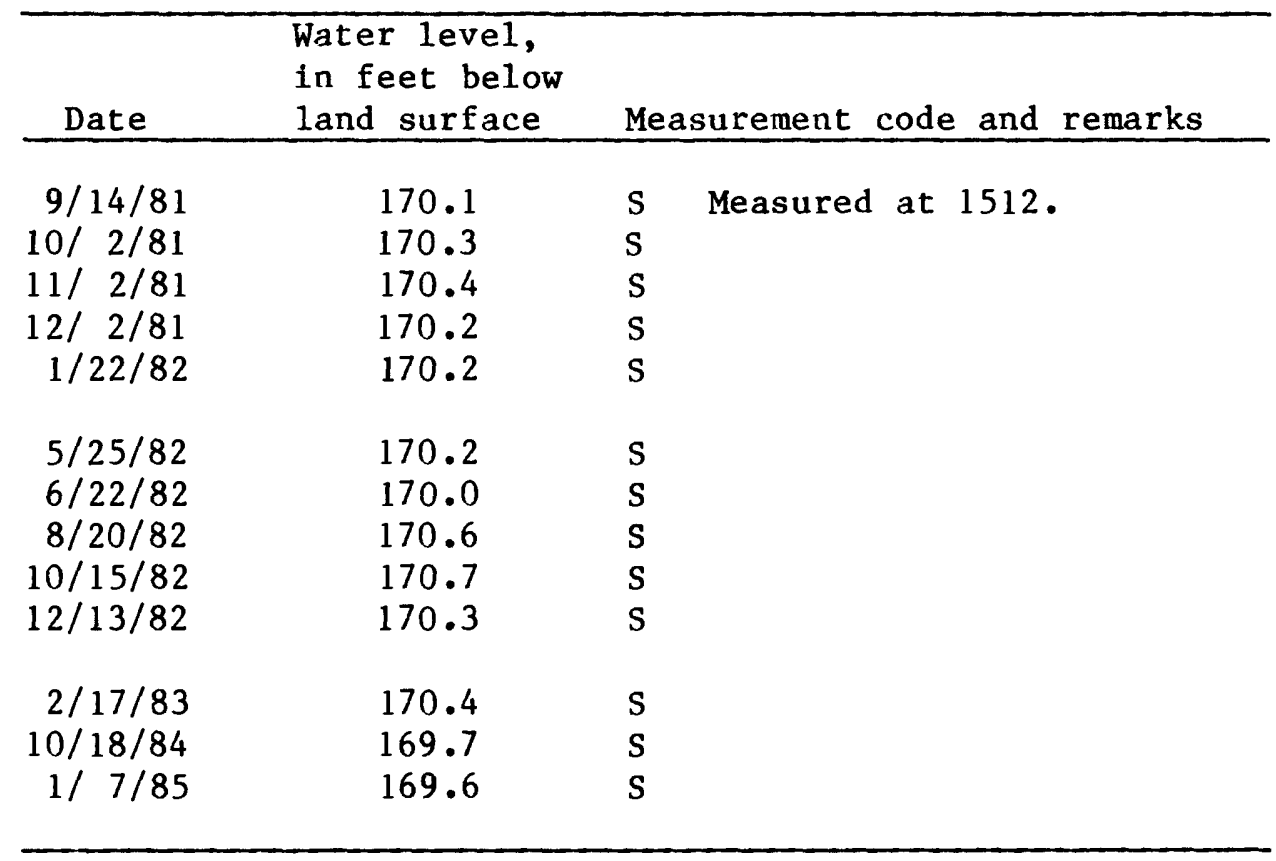


Table 26. Water-level records for well H-7C, Rustler FormationSalado Formation contact zone

[Measurement code: A, Air-1ine measurement; L, Lynes Pressure Sentry System; M, M-Scope; S, Steel tape; W, Winch. Total depth is in feet below land surface. Land-surface altitude is in feet above sea level.]

Location: $23 \cdot 30 \cdot 14 \cdot 2592 \mathrm{FNL} \cdot 2468 \mathrm{FWL}$

Total depth: $\quad 420.0$ Land-surface altitude: $\quad 3,163.5$

\begin{tabular}{|c|c|c|}
\hline Date & $\begin{array}{l}\text { Water level, } \\
\text { in feet below } \\
\text { land surface }\end{array}$ & Measurement code and remarks \\
\hline $9 / 19 / 79$ & 199 & $\mathrm{~W}$ \\
\hline $9 / 26 / 79$ & 199 & W \\
\hline $10 / 10 / 79$ & 199 & W \\
\hline $4 / 15 / 80$ & 199 & W \\
\hline $5 / 6 / 80$ & 199 & $\mathrm{~W}$ \\
\hline $6 / 10 / 80$ & 198.7 & S \\
\hline $7 / 1 / 80$ & 198.6 & S \\
\hline $8 / 2 / 80$ & 198.6 & $\mathrm{~S}$ \\
\hline $9 / 8 / 80$ & 198.5 & S \\
\hline $10 / 8 / 80$ & 198.4 & S \\
\hline $11 / 4 / 80$ & 198.3 & S \\
\hline $11 / 24 / 80$ & 214 & W \\
\hline $11 / 25 / 80$ & 213.3 & S \\
\hline $12 / 2 / 80$ & 214.9 & $\mathrm{~S}$ \\
\hline $2 / 4 / 81$ & 212.0 & $\mathrm{~S}$ \\
\hline $5 / 4 / 81$ & 206.6 & S \\
\hline $6 / 2 / 81$ & 206.4 & S \\
\hline $7 / 6 / 81$ & 206.3 & S \\
\hline $7 / 23 / 81$ & 206.3 & S \\
\hline $8 / 4 / 81$ & 206.2 & $S$ \\
\hline $9 / 14 / 81$ & 205.9 & S \\
\hline $9 / 16 / 81$ & 205.9 & $\mathrm{~S}$ \\
\hline $10 / 2 / 81$ & 205.7 & $\mathrm{~S}$ \\
\hline $11 / 2 / 81$ & 205.5 & $\mathrm{~s}$ \\
\hline $12 / 2 / 81$ & 205.2 & S \\
\hline $1 / 22 / 82$ & 204.8 & $\mathrm{~s}$ \\
\hline $5 / 15 / 82$ & 204.1 & S \\
\hline $6 / 22 / 82$ & 203.9 & S \\
\hline $8 / 20 / 82$ & 203.8 & $S$ \\
\hline $10 / 15 / 82$ & 203.7 & $S$ \\
\hline $12 / 13 / 82$ & 203.3 & S \\
\hline $2 / 17 / 83$ & 203.1 & $\mathrm{~S}$ \\
\hline
\end{tabular}


Table 27. Water-level records for well H-8A, Magenta Dolomite Member of the Rustler Formation

[Measurement code: A, Air-line measurement; L, Lynes Pressure Sentry System; M, M-Scope; S, Steel tape; W, Winch. Total depth is in feet below land surface. Land-surface altitude is in feet above sea level.]

Location: $24.30 .23 .1963 \mathrm{FNL} .1487 \mathrm{FEL}$

Total depth: $\quad 505.0$ Land-surface altitude: $\quad 3,433.0$

\begin{tabular}{ccc}
\hline & $\begin{array}{c}\text { Water level, } \\
\text { in feet below } \\
\text { 1and surface }\end{array}$ & Measurement code and remarks \\
\hline $11 / 1 / 79$ & 456 & W \\
$12 / 4 / 79$ & 420.5 & S \\
$1 / 2 / 80$ & 411.3 & S \\
$1 / 28 / 80$ & 407.0 & S \\
$2 / 15 / 80$ & 429 & W \\
& & \\
$3 / 2 / 80$ & 421 & W \\
$4 / 16 / 80$ & 411 & W \\
$5 / 6 / 80$ & 410 & W \\
$6 / 10 / 80$ & 408 & W \\
$7 / 1 / 80$ & 407 & W \\
$8 / 9 / 80$ & 406.0 & S \\
$9 / 6 / 80$ & 405.7 & S \\
$10 / 7 / 80$ & 405.4 & S \\
$11 / 5 / 80$ & 405.2 & S \\
$12 / 3 / 80$ & 405.1 & S \\
$2 / 6 / 81$ & 405.0 & S \\
$5 / 4 / 81$ & 405.0 & S \\
$7 / 7 / 81$ & 405.0 & S \\
$10 / 2 / 81$ & 405.1 & S \\
$1 / 22 / 82$ & 405.2 & S \\
$5 / 25 / 82$ & 405.2 & S \\
$6 / 22 / 82$ & 405.2 & S \\
$8 / 20 / 82$ & 405.3 & S \\
$10 / 15 / 82$ & 405.4 & S \\
$12 / 13 / 82$ & 405.4 & S \\
$2 / 17 / 83$ & 405.3 & S \\
$8 / 24 / 83$ & 405.3 & S \\
$1 / 24 / 84$ & 405.3 & S \\
$1 / 20 / 84$ & 405.2 & S \\
& 405.2 & S \\
\hline
\end{tabular}


Table 28. Water-level records for well H-8B, Culebra Dolomite Member of the Rustler Formation

[Measurement code: A, Air-line measurement; L, Lynes Pressure Sentry System; M, M-Scope; S, Steel tape; W, Winch. Total depth is in feet below land surface. Land-surface altitude is in feet above sea level.]

Location: $24 \cdot 30 \cdot 23 \cdot 1995 \mathrm{FNL} .1405 \mathrm{FEL}$

Total depth: $\quad 624.0$ Land-surface altitude: $\quad 3,433.8$

\begin{tabular}{|c|c|c|c|}
\hline Date & $\begin{array}{l}\text { Water level, } \\
\text { in feet below } \\
\text { land surface }\end{array}$ & Measurement code & and remarks \\
\hline $\begin{array}{l}8 / 13 / 79 \\
8 / 15 / 79 \\
8 / 17 / 79 \\
8 / 20 / 79 \\
8 / 23 / 79\end{array}$ & $\begin{array}{l}452 \\
450 \\
451 \\
449.6 \\
448.8\end{array}$ & $\begin{array}{l}W \\
W \\
W \\
S \\
S\end{array}$ & \\
\hline $\begin{array}{r}8 / 28 / 79 \\
9 / 7 / 79 \\
10 / 10 / 79 \\
11 / 1 / 79 \\
12 / 4 / 79\end{array}$ & $\begin{array}{l}441.2 \\
450 \\
450 \\
449.1 \\
449.3\end{array}$ & $\begin{array}{l}S \\
W \\
W \\
S \\
S\end{array}$ & \\
\hline $\begin{array}{l}1 / 2 / 80 \\
1 / 28 / 80 \\
1 / 30 / 80 \\
2 / 1 / 80 \\
2 / 2 / 80\end{array}$ & $\begin{array}{l}449.7 \\
448.3 \\
448.5 \\
448.8 \\
448.5\end{array}$ & $\begin{array}{l}S \\
S \\
S \\
S \\
S\end{array}$ & . \\
\hline $\begin{array}{ll}2 / & 3 / 80 \\
2 / & 4 / 80 \\
2 / & 5 / 80 \\
2 / & 6 / 80 \\
2 / 11 / 80\end{array}$ & $\begin{array}{l}448.6 \\
448.4 \\
448.6 \\
448.3 \\
448.4\end{array}$ & $\begin{array}{l}S \\
S \\
S \\
S \\
S\end{array}$ & \\
\hline $\begin{array}{l}3 / 2 / 80 \\
4 / 16 / 80 \\
5 / 6 / 80 \\
6 / 10 / 80 \\
7 / 1 / 80\end{array}$ & $\begin{array}{l}449 \\
448 \\
447 \\
448 \\
448\end{array}$ & $\begin{array}{l}W \\
W \\
W \\
W \\
W\end{array}$ & \\
\hline $\begin{aligned} 8 / & 9 / 80 \\
9 / & 6 / 80 \\
10 / & 7 / 80 \\
11 / & 5 / 80 \\
12 / & 3 / 80\end{aligned}$ & $\begin{array}{l}446.2 \\
446.5 \\
445.8 \\
445.3 \\
445.3\end{array}$ & $\begin{array}{l}S \\
S \\
S \\
S \\
S\end{array}$ & \\
\hline
\end{tabular}


Table 28. Water-level records for well H-8B, Culebra Dolomite Member of the Rustler Formation - Concluded

\begin{tabular}{rrr}
\hline & $\begin{array}{l}\text { Water level, } \\
\text { in feet below } \\
\text { Date }\end{array}$ & \\
\hline land surface & Measurement code and remarks \\
$2 / 6 / 81$ & 444.7 & $\mathrm{~S}$ \\
$5 / 4 / 81$ & 444.5 & $\mathrm{~S}$ \\
$7 / 7 / 81$ & 444.5 & $\mathrm{~S}$ \\
$10 / 2 / 81$ & 443.7 & $\mathrm{~S}$ \\
$1 / 22 / 82$ & 442.6 & $\mathrm{~S}$ \\
& & \\
$5 / 25 / 82$ & 442.2 & $\mathrm{~S}$ \\
$6 / 22 / 82$ & 442.3 & $\mathrm{~S}$ \\
$8 / 20 / 82$ & 443.0 & $\mathrm{~S}$ \\
$10 / 15 / 82$ & 442.1 & $\mathrm{~S}$ \\
$12 / 13 / 82$ & 441.1 & $\mathrm{~S}$ \\
& & \\
$2 / 17 / 83$ & 440.6 & $\mathrm{~S}$ \\
$8 / 24 / 83$ & 441.2 & $\mathrm{~S}$ \\
$1 / 24 / 84$ & 440.9 & $\mathrm{~S}$ \\
$10 / 20 / 84$ & 440.4 & $\mathrm{~S}$ \\
$1 / 7 / 85$ & 440.3 & $\mathrm{~S}$ \\
& & \\
\hline
\end{tabular}


Table 29. Water-level records for well H-8C, Rustler FormationSalado Formation contact zone

[Measurement code: A, Air-line measurement; L, Lynes Pressure Sentry System; M, M-Scope; S, Steel tape; W, Winch. Total depth is in feet below land surface. Land-surface altitude is in feet above sea level.]

Location: $24 \cdot 30 \cdot 23.2059 \mathrm{FNL} .1470 \mathrm{FEL}$

Total depth: $\quad 808.0$ Land-surface altitude: $\quad 3,433.0$

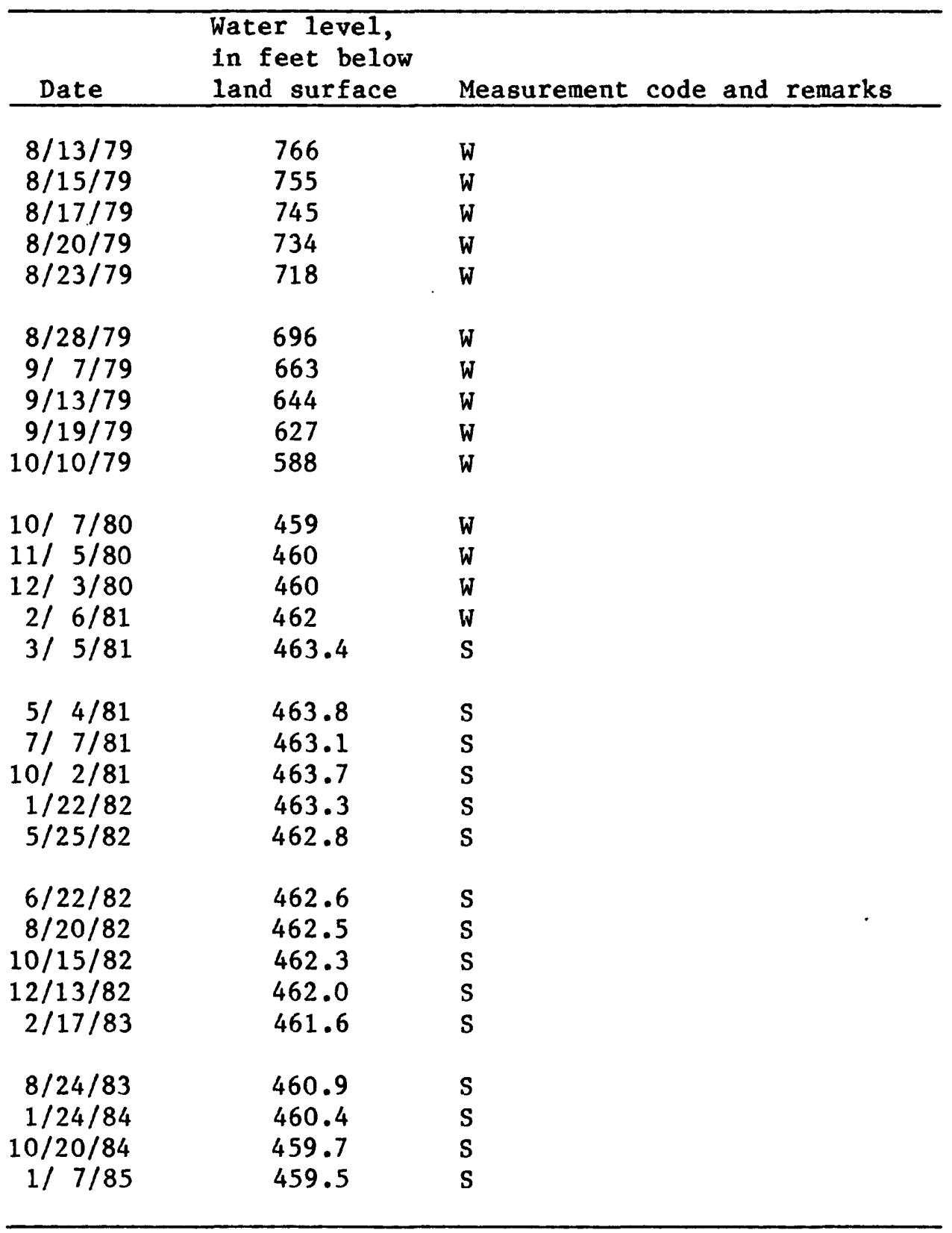


Table 30. Water-level records for well H-9A, Magenta Dolomite Member of the Rustler Formation

[Measurement code: A, Air-line measurement; L, Lynes Pressure Sentry System; M, M-Scope; S, Steel tape; W, Winch. Total depth is in feet below land surface. Land-surface altitude is in feet above sea level.]

Location: $24.31 .04 .2392 \mathrm{FNL} .0139 \mathrm{FWL}$

Total depth: $\quad 559.0$ Land-surface altitude: $\quad 3,404.4$

\begin{tabular}{|c|c|c|}
\hline Date & $\begin{array}{l}\text { Water level, } \\
\text { in feet below } \\
\text { land surface }\end{array}$ & Measurement code and remarks \\
\hline $\begin{array}{r}9 / 7 / 79 \\
9 / 13 / 79 \\
9 / 26 / 79 \\
10 / 10 / 79 \\
11 / 1 / 79\end{array}$ & $\begin{array}{l}297 \\
289 \\
284 \\
284 \\
284.4\end{array}$ & $\begin{array}{l}W \\
W \\
W \\
W \\
S\end{array}$ \\
\hline $\begin{array}{rr}12 / & 4 / 79 \\
1 / & 2 / 80 \\
2 / & 1 / 80 \\
2 / & 6 / 80 \\
2 / 1 & 3 / 80\end{array}$ & $\begin{array}{l}284.1 \\
283.6 \\
283.7 \\
289 \\
284\end{array}$ & $\begin{array}{l}S \\
S \\
S \\
W \\
W\end{array}$ \\
\hline $\begin{array}{l}3 / 2 / 80 \\
4 / 16 / 80 \\
5 / 6 / 80 \\
6 / 10 / 80 \\
7 / 1 / 80\end{array}$ & $\begin{array}{l}284 \\
283 \\
283 \\
284 \\
283\end{array}$ & $\begin{array}{l}W \\
W \\
W \\
W \\
W\end{array}$ \\
\hline 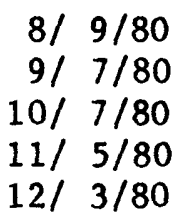 & $\begin{array}{l}283.0 \\
283.1 \\
282.8 \\
282.8 \\
282.7\end{array}$ & $\begin{array}{l}S \\
S \\
S \\
S \\
S\end{array}$ \\
\hline 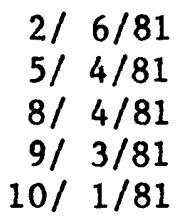 & $\begin{array}{l}282.0 \\
282.2 \\
282.4 \\
282.4 \\
282.5\end{array}$ & $\begin{array}{l}S \\
S \\
S \\
S \\
S\end{array}$ \\
\hline $\begin{array}{r}11 / 2 / 81 \\
12 / 2 / 81 \\
1 / 21 / 82 \\
2 / 18 / 82 \\
3 / 10 / 82\end{array}$ & $\begin{array}{l}282.5 \\
282.4 \\
282.3 \\
282.3 \\
281.0\end{array}$ & $\begin{array}{l}S \\
S \\
S \\
S \\
S\end{array}$ \\
\hline
\end{tabular}


Table 30. Water-level records for well H-9A, Magenta Dolomite Member of the Rustler Formation - Concluded

\begin{tabular}{|c|c|c|}
\hline Date & $\begin{array}{l}\text { Water level, } \\
\text { In feet below } \\
\text { land surface }\end{array}$ & Measurement code and remarks \\
\hline $\begin{array}{r}4 / 15 / 82 \\
5 / 17 / 82 \\
6 / 22 / 82 \\
9 / 2 / 82 \\
10 / 29 / 82\end{array}$ & $\begin{array}{l}282.1 \\
282.1 \\
282.3 \\
282.3 \\
282.3\end{array}$ & $\begin{array}{l}S \\
\text { S } \\
\text { S } \\
\text { S } \\
\text { S }\end{array}$ \\
\hline $\begin{array}{r}12 / 13 / 82 \\
2 / 17 / 83\end{array}$ & $\begin{array}{l}282.0 \\
282.0\end{array}$ & $\begin{array}{l}S \\
S\end{array}$ \\
\hline
\end{tabular}


Table 31. Water-level records for well $\mathrm{H}-9 \mathrm{~B}$, Culebra Dolomite Member of the Rustler Formation

[Measurement code: A, Air-line measurement; L, Lynes Pressure Sentry System; M, M-Scope; S, Steel tape; W, Winch. Total depth is in feet below land surface. Land-surface altitude is in feet above sea level.]

Location: $24.31 .04 .2391 \mathrm{FNL} .0239 \mathrm{FWL}$

Total depth: $\quad 708.0$ Land-surface altitude: $\quad 3,404.7$

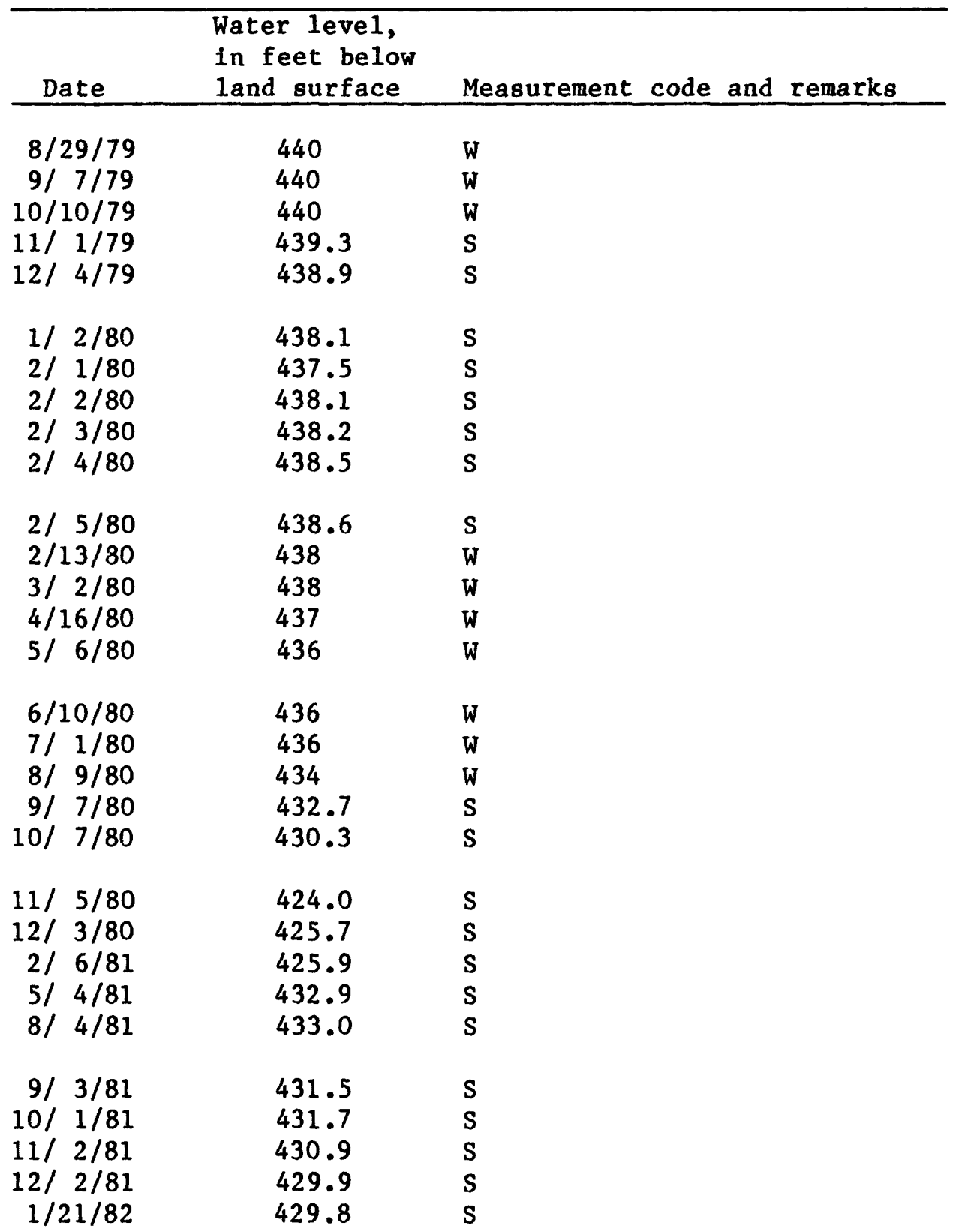


Table 31. Water-level records for well $\mathrm{H}-9 \mathrm{~B}$, Culebra Dolomite Member of the Rustler Formation - Concluded

\begin{tabular}{ccc}
\hline & $\begin{array}{l}\text { Water level, } \\
\text { 1n feet below } \\
\text { Date }\end{array}$ & \\
land surface & Measurement code and remarks \\
\hline $2 / 18 / 82$ & 429.2 & $\mathrm{~S}$ \\
$3 / 10 / 82$ & 428.1 & $\mathrm{~S}$ \\
$4 / 15 / 82$ & 428.3 & $\mathrm{~S}$ \\
$6 / 22 / 82$ & 429.4 & $\mathrm{~S}$ \\
$9 / 2 / 82$ & 429.4 & $\mathrm{~S}$ \\
& & \\
$10 / 29 / 82$ & 431.9 & $\mathrm{~S}$ \\
$12 / 13 / 82$ & 428.2 & $\mathrm{~S}$ \\
$2 / 17 / 83$ & 428.7 & $\mathrm{~S}$ \\
$10 / 20 / 84$ & 430.3 & $\mathrm{~S}$ \\
$1 / 7 / 85$ & 430.7 & $\mathrm{~S}$ \\
\hline
\end{tabular}


Table 32. Water-level records for well H-9C, Rustler FormationSalado Formation contact zone

[Measurement code: A, Alr-11ne measurement; L, Lynes Pressure Sentry System; M, M-Scope; S, Steel tape; W, Winch. Total depth is in feet below land surface. Land-surface altitude is in feet above sea level.]

Location: $\quad 24.31 .04 .2479 \mathrm{FNL} .0188 \mathrm{FWL}$

Total depth: $\quad 816.0$ Land-surface altitude: $\quad 3,405.9$

\begin{tabular}{rcc}
\hline & $\begin{array}{c}\text { Water level, } \\
\text { in feet below } \\
\text { land surface }\end{array}$ & Measurement code and remarks \\
\hline Date & 788 & W \\
$8 / 17 / 79$ & 788 & W \\
$8 / 20 / 79$ & 788 & W \\
$8 / 23 / 79$ & 788 & W \\
$8 / 28 / 79$ & 815 & W \\
$9 / 27 / 79$ & & \\
& 816 & W \\
$10 / 10 / 79$ & 809 & W \\
$2 / 13 / 80$ & 809 & W \\
$2 / 15 / 80$ & 809 & W \\
$2 / 16 / 80$ & 809 & W \\
$2 / 27 / 80$ & & \\
$3 / 2 / 80$ & 808 & W \\
$4 / 16 / 80$ & 804 & W \\
$5 / 6 / 80$ & 803 & W \\
$5 / 5 / 81$ & 728 & W \\
$6 / 2 / 81$ & 714 & W \\
$8 / 4 / 81$ & 713 & W \\
$9 / 3 / 81$ & 704 & W \\
$10 / 1 / 81$ & 690 & W \\
$12 / 2 / 81$ & 680.6 & S \\
$1 / 21 / 82$ & 665 & W \\
$2 / 18 / 82$ & 656.3 & S \\
$3 / 10 / 82$ & 656.5 & S \\
$8 / 19 / 82$ & 715.2 & S \\
$1 / 19 / 83$ & 708 & W Measured after pulling \\
$2 / 1 / 83$ & 717 & W packer. \\
& & \\
\hline
\end{tabular}


Table 33. Water-level records for well H-10A, Magenta Dolomite Member of the Rustler Formation

[Measurement code: A, Air-line measurement; L, Lynes Pressure Sentry System; M, M-Scope; S, Steel tape; W, Winch. Total depth is in feet below land surface. Land-surface altitude is in feet above sea level.]

Location: $23.32 \cdot 20.0433 \mathrm{FSL} .2069 \mathrm{FEL}$

Total depth: $\quad 1,318.0$ Land-surface altitude: $\quad 3,686.6$

\begin{tabular}{|c|c|c|}
\hline Date & $\begin{array}{l}\text { Water level, } \\
\text { in feet below } \\
\text { land surface }\end{array}$ & Measurement code and remarks \\
\hline $\begin{array}{l}8 / 28 / 79 \\
9 / 7 / 79 \\
9 / 13 / 79 \\
9 / 18 / 79 \\
9 / 26 / 79\end{array}$ & $\begin{array}{r}1,142 \\
847 \\
758 \\
711 \\
665\end{array}$ & $\begin{array}{l}W \\
W \\
W \\
W \\
W\end{array}$ \\
\hline $\begin{array}{l}10 / 10 / 79 \\
11 / 1 / 79 \\
11 / 21 / 79 \\
11 / 26 / 79 \\
12 / 3 / 79\end{array}$ & $\begin{array}{l}625 \\
602 \\
594 \\
593 \\
592\end{array}$ & $\begin{array}{l}\text { W } \\
W \\
W \\
W \\
W\end{array}$ \\
\hline $\begin{array}{r}12 / 10 / 79 \\
2 / 1 / 80 \\
2 / 13 / 80 \\
2 / 15 / 80 \\
2 / 16 / 80\end{array}$ & $\begin{array}{l}592 \\
590 \\
589 \\
589 \\
589\end{array}$ & $\begin{array}{l}W \\
W \\
W \\
W \\
W\end{array}$ \\
\hline $\begin{array}{l}2 / 17 / 80 \\
3 / 2 / 80 \\
4 / 16 / 80 \\
5 / 6 / 80 \\
6 / 10 / 80\end{array}$ & $\begin{array}{l}587 \\
670 \\
619 \\
600 \\
591\end{array}$ & $\begin{array}{l}\text { W } \\
W \\
W \\
W \\
W\end{array}$ \\
\hline 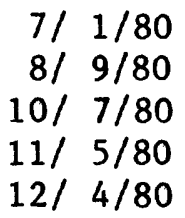 & $\begin{array}{l}591 \\
590 \\
589 \\
589 \\
588\end{array}$ & $\begin{array}{l}W \\
W \\
W \\
W \\
W\end{array}$ \\
\hline $\begin{aligned} 2 / & 6 / 81 \\
5 / & 5 / 81 \\
8 / & 4 / 81 \\
10 / & 1 / 81 \\
11 / & 2 / 81\end{aligned}$ & $\begin{array}{l}587.2 \\
586.8 \\
586.8 \\
586.8 \\
586.7\end{array}$ & $\begin{array}{l}\mathrm{S} \\
\mathrm{S} \\
\mathrm{S} \\
\mathrm{S} \\
\mathrm{S}\end{array}$ \\
\hline
\end{tabular}


Table 33. Water-level records for well H-10A, Magenta Dolomite Member of the Rustler Formation - Concluded

\begin{tabular}{|c|c|c|}
\hline Date & $\begin{array}{l}\text { Water level, } \\
\text { in feet below } \\
\text { land surface }\end{array}$ & Measurement code and remarks \\
\hline $\begin{array}{r}12 / 2 / 81 \\
1 / 21 / 82 \\
2 / 18 / 82 \\
3 / 10 / 82 \\
4 / 15 / 82\end{array}$ & $\begin{array}{l}586.7 \\
586.6 \\
586.5 \\
586.5 \\
586.4\end{array}$ & $\begin{array}{l}S \\
S \\
S \\
S \\
S\end{array}$ \\
\hline $\begin{array}{l}5 / 26 / 82 \\
8 / 20 / 82\end{array}$ & $\begin{array}{l}586.4 \\
586.6\end{array}$ & $\begin{array}{l}S \\
S\end{array}$ \\
\hline
\end{tabular}


Table 34. Water-level records for well $\mathrm{H}-10 \mathrm{~B}$, Culebra Dolomite Member of the Rustler Formation

[Measurement code: A, Air-1ine measurement; L, Lynes Pressure Sentry System; M, M-Scope; S, Steel tape; W, Winch. Total depth is in feet below land surface. Land-surface altitude is in feet above sea level.]

Location: 23.32 .20 .0485 FSL.1985FEL

Total depth: $\quad 1,398.0$ Land-surface altitude: $\quad 3,687.0$

\begin{tabular}{|c|c|c|}
\hline Date & $\begin{array}{l}\text { Water level, } \\
\text { in feet below } \\
\text { land surface }\end{array}$ & Measurement code and remarks \\
\hline $\begin{array}{l}11 / 1 / 79 \\
11 / 21 / 79 \\
11 / 26 / 79 \\
12 / 3 / 79 \\
12 / 10 / 79\end{array}$ & $\begin{array}{l}695 \\
689 \\
689 \\
688 \\
688\end{array}$ & $\begin{array}{l}W \\
W \\
W \\
W \\
W\end{array}$ \\
\hline $\begin{array}{l}2 / 1 / 80 \\
2 / 13 / 80 \\
2 / 15 / 80 \\
2 / 16 / 80 \\
2 / 21 / 80\end{array}$ & $\begin{array}{l}687 \\
687 \\
686 \\
686 \\
687\end{array}$ & $\begin{array}{l}W \\
W \\
W \\
W \\
W\end{array}$ \\
\hline $\begin{array}{l}2 / 25 / 80 \\
3 / 2 / 80 \\
4 / 16 / 80 \\
5 / 6 / 80 \\
6 / 10 / 80\end{array}$ & $\begin{array}{l}684 \\
718 \\
687 \\
699 \\
697\end{array}$ & $\begin{array}{l}W \\
W \\
W \\
W \\
W\end{array}$ \\
\hline $\begin{array}{rr}7 / & 1 / 80 \\
8 / & 9 / 80 \\
10 / & 7 / 80 \\
11 / & 5 / 80 \\
12 / & 4 / 80\end{array}$ & $\begin{array}{l}699 \\
699 \\
699 \\
699 \\
700\end{array}$ & $\begin{array}{l}W \\
W \\
W \\
W \\
W\end{array}$ \\
\hline $\begin{aligned} 2 / & 6 / 81 \\
5 / & 5 / 81 \\
8 / & 4 / 81 \\
10 / & 1 / 81 \\
11 / & 2 / 81\end{aligned}$ & $\begin{array}{l}697.9 \\
697.7 \\
697.8 \\
697.8 \\
691.3\end{array}$ & $\begin{array}{l}\text { S } \\
\text { S } \\
\text { S } \\
\text { S } \\
\text { S }\end{array}$ \\
\hline
\end{tabular}


Table 34. Water-level records for well H-10B, Culebra Dolomite Member of the Rustler Formation - Concluded

\begin{tabular}{|c|c|c|}
\hline Date & $\begin{array}{l}\text { Water level, } \\
\text { in feet below } \\
\text { land surface }\end{array}$ & Measurement code and remarks \\
\hline $\begin{array}{r}12 / 2 / 81 \\
1 / 21 / 82 \\
2 / 18 / 82 \\
3 / 10 / 82 \\
4 / 15 / 82\end{array}$ & $\begin{array}{l}687.1 \\
699 \\
696.4 \\
697.3 \\
697.0\end{array}$ & $\begin{array}{l}S \\
W \\
S \\
S \\
S\end{array}$ \\
\hline $\begin{array}{l}5 / 26 / 82 \\
8 / 20 / 82\end{array}$ & $\begin{array}{l}697.0 \\
697.2\end{array}$ & $\begin{array}{l}S \\
S\end{array}$ \\
\hline
\end{tabular}


Table 35. Water-level records for well H-10C, Rustler FormationSalado Formation contact zone

[Measurement code: A, Air-line measurement; L, Lynes Pressure Sentry System; M, M-Scope; S, Steel tape; W, Winch. Total depth is in feet below land surface. Land-surface altitude is in feet above sea level.]

Location: $23.32 \cdot 20.0385 \mathrm{FSL} .1982 \mathrm{FEL}$

Total depth: $\quad 1,538.0$ Land-surface altitude: $\quad 3,686.9$

\begin{tabular}{|c|c|c|}
\hline Date & $\begin{array}{l}\text { Water level, } \\
\text { in feet below } \\
\text { land surface }\end{array}$ & Measurement code and remarks \\
\hline $\begin{array}{r}8 / 23 / 79 \\
8 / 28 / 79 \\
9 / 7 / 79 \\
9 / 26 / 79 \\
10 / 10 / 79\end{array}$ & $\begin{array}{l}1,517 \\
1,517 \\
1,517 \\
1,516 \\
1,516\end{array}$ & $\begin{array}{l}W \\
W \\
W \\
W \\
W\end{array}$ \\
\hline $\begin{array}{l}2 / 17 / 80 \\
2 / 27 / 80 \\
3 / 2 / 80 \\
4 / 16 / 80 \\
5 / 6 / 80\end{array}$ & $\begin{array}{l}1,507 \\
1,508 \\
1,514 \\
1,498 \\
1,498\end{array}$ & $\begin{array}{l}W \\
W \\
W \\
W \\
W\end{array}$ \\
\hline 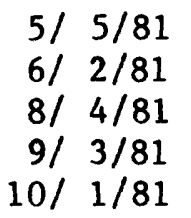 & $\begin{array}{l}1,402 \\
1,389 \\
1,375 \\
1,369 \\
1,365\end{array}$ & $\begin{array}{l}W \\
W \\
W \\
W \\
W\end{array}$ \\
\hline $\begin{array}{l}1 / 21 / 82 \\
5 / 26 / 82 \\
8 / 20 / 82 \\
1 / 19 / 83\end{array}$ & $\begin{array}{l}1,346 \\
1,328 \\
1,315 \\
1,292\end{array}$ & $\begin{array}{l}W \\
W \\
W \\
W\end{array}$ \\
\hline
\end{tabular}


Table 36. Water-level records for well P-14, Culebra Dolomite Member of the Rustler Formation

[Measurement code: A, Alr-1ine measurement; L, Lynes Pressure Sentry System; M, M-Scope; S, Steel tape; W, Winch. Total depth is in feet below land surface. Land-surface altitude is in feet above sea level.]

Location: $22 \cdot 30.24 .0309 \mathrm{FSL} .0613 \mathrm{FWL}$

Total depth: $1,545.0$ Land-surface altitude: $3,360.3$

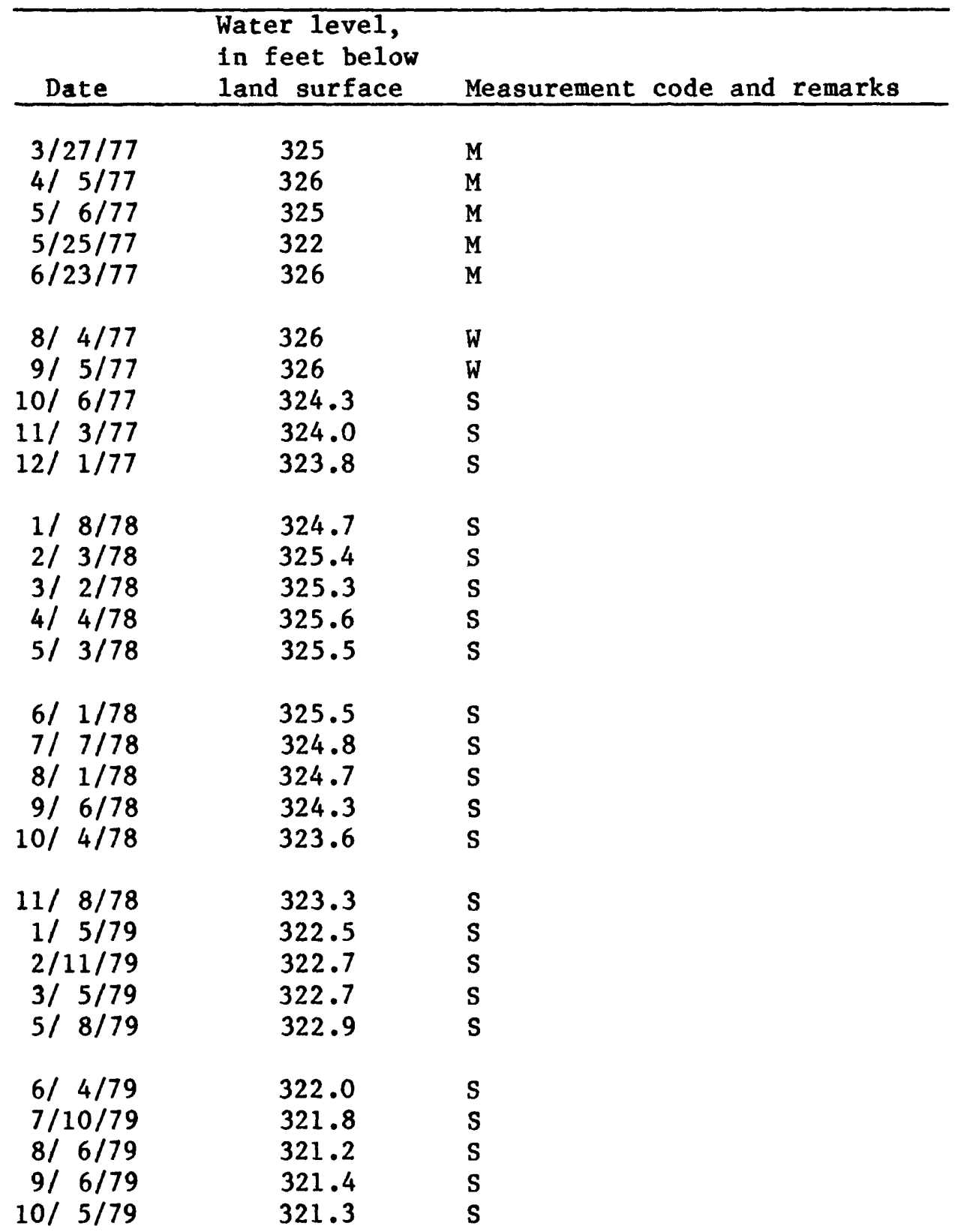


Table 36. Water-level records for well $\mathrm{P}-14$, Culebra Dolomite Member of the Rustler Formation - Concluded

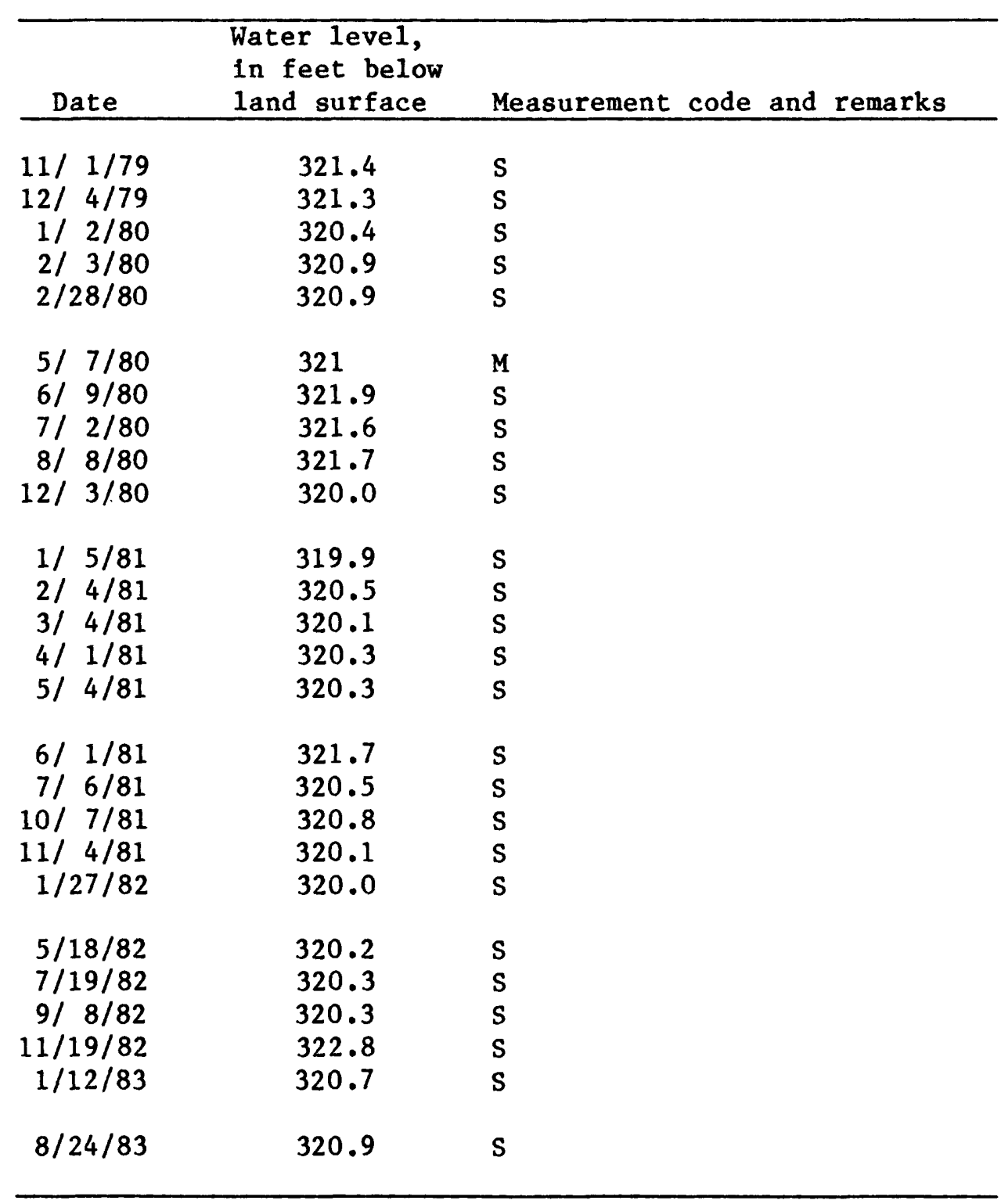


Table 37. Water-level records for well $\mathrm{P}-14$, Rustler FormationSalado Formation contact zone

[Measurement code: A, Alr-11ne measurement; L, Lynes Pressure Sentry System; M, M-Scope; S, Steel tape; W, Winch. Total depth is in feet below land surface. Land-surface altitude is in feet above sea level.]

Location: $22 \cdot 30 \cdot 24.0309 \mathrm{FSL} .0613 \mathrm{FWL}$

Total depth: $\quad 1,545.0$ Land-surface altitude: $3,360.3$

\begin{tabular}{|c|c|c|}
\hline Date & $\begin{array}{l}\text { Water level, } \\
\text { in feet below } \\
\text { land surface }\end{array}$ & Measurement code and remarks \\
\hline $\begin{array}{l}3 / 24 / 77 \\
3 / 25 / 77 \\
3 / 26 / 77 \\
3 / 27 / 77 \\
4 / 5 / 77\end{array}$ & $\begin{array}{l}457 \\
403 \\
389 \\
386 \\
385\end{array}$ & $\begin{array}{l}M \\
M \\
M \\
M \\
M\end{array}$ \\
\hline $\begin{array}{l}5 / 6 / 77 \\
5 / 25 / 77 \\
6 / 23 / 77 \\
8 / 4 / 77 \\
9 / 5 / 77\end{array}$ & $\begin{array}{l}386 \\
386 \\
386.2 \\
389 \\
383\end{array}$ & $\begin{array}{l}M \\
M \\
S \\
M \\
M\end{array}$ \\
\hline $\begin{aligned} 10 / & 6 / 77 \\
11 / & 3 / 77 \\
12 / & 1 / 77 \\
1 / & 8 / 78 \\
2 / & 3 / 78\end{aligned}$ & $\begin{array}{l}386.2 \\
386.2 \\
386.1 \\
386.2 \\
386.2\end{array}$ & $\begin{array}{l}S \\
S \\
S \\
S \\
S\end{array}$ \\
\hline $\begin{array}{ll}3 / & 2 / 78 \\
4 / & 4 / 78 \\
5 / & 3 / 78 \\
6 / & 1 / 78 \\
7 / & 7 / 78\end{array}$ & $\begin{array}{l}386.0 \\
385.9 \\
386.0 \\
386.0 \\
386.0\end{array}$ & $\begin{array}{l}\text { S } \\
\text { S } \\
\text { S } \\
\text { S } \\
\text { S }\end{array}$ \\
\hline $\begin{aligned} 8 / & 1 / 78 \\
9 / & 6 / 78 \\
10 / & 4 / 78 \\
11 / & 8 / 78 \\
1 / & 5 / 79\end{aligned}$ & $\begin{array}{l}386.0 \\
385.8 \\
385.7 \\
385.6 \\
385.6\end{array}$ & $\begin{array}{l}\text { S } \\
S \\
S \\
S \\
S\end{array}$ \\
\hline 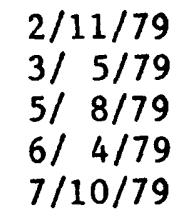 & $\begin{array}{l}386.2 \\
385.5 \\
385.4 \\
385.4 \\
385.5\end{array}$ & $\begin{array}{l}S \\
S \\
S \\
S \\
S\end{array}$ \\
\hline
\end{tabular}


Table 37. Water-level records for well P-14, Rustler FormationSalado Formation contact zone - Concluded

\begin{tabular}{rcl} 
& \multicolumn{1}{l}{$\begin{array}{c}\text { Water level, } \\
\text { in feet below }\end{array}$} \\
Date & land surface & Measurement code and remarks \\
\hline & & \\
$8 / 6 / 79$ & 385.6 & $\mathrm{~S}$ \\
$9 / 6 / 79$ & 385.5 & $\mathrm{~S}$ \\
$10 / 5 / 79$ & 385.5 & $\mathrm{~S}$ \\
$11 / 1 / 79$ & 385.5 & $\mathrm{~S}$ \\
$12 / 4 / 79$ & 385.5 & $\mathrm{~S}$ \\
& & \\
$1 / 2 / 80$ & 385.3 & $\mathrm{~S}$ \\
$1 / 31 / 80$ & 385.1 & $\mathrm{~S}$ \\
$2 / 3 / 80$ & 385.4 & $\mathrm{~S}$ \\
$2 / 28 / 80$ & 386.7 & $\mathrm{~S}$ \\
$9 / 8 / 80$ & 359 & $\mathrm{M}$ \\
$9 / 16 / 80$ & 360 & $\mathrm{M}$ \\
$10 / 3 / 80$ & 388 & $\mathrm{M}$ \\
$11 / 4 / 80$ & 389.2 & $\mathrm{~S}$ \\
$12 / 3 / 80$ & 388.9 & $\mathrm{~S}$ \\
$1 / 5 / 81$ & 388.9 & $\mathrm{~S}$ \\
& & \\
$2 / 4 / 81$ & 389.0 & $\mathrm{~S}$ \\
$3 / 4 / 81$ & 388.7 & $\mathrm{~S}$ \\
$4 / 1 / 81$ & 388.9 & $\mathrm{~S}$ \\
$5 / 4 / 81$ & 388.7 & $\mathrm{~S}$ \\
$6 / 1 / 81$ & 388.8 & $\mathrm{~S}$ \\
$7 / 6 / 81$ & 388.9 & $\mathrm{~S}$ \\
$10 / 7 / 81$ & 389.0 & $\mathrm{~S}$ \\
$11 / 4 / 81$ & 388.9 & $\mathrm{~S}$ \\
$1 / 27 / 82$ & 388.9 & $\mathrm{~S}$ \\
$5 / 18 / 82$ & 388.6 & $\mathrm{~S}$ \\
$7 / 19 / 82$ & 388.8 & $\mathrm{~S}$ \\
$9 / 8 / 82$ & 388.4 & $\mathrm{~S}$ \\
$11 / 19 / 82$ & 388.2 & $\mathrm{~S}$ \\
$1 / 12 / 83$ & 388.2 & $\mathrm{~S}$ \\
$8 / 24 / 83$ & 387.7 & $\mathrm{~S}$ \\
& & \\
\hline
\end{tabular}


Table 38. Water-level records for well P-15, Culebra Dolomite Member of the Rustler Formation

[Measurement code: A, Air-line measurement; L, Lynes Pressure Sentry System; M, M-Scope; S, Steel tape; W, Winch. Total depth is in feet below land surface. Land-surface altitude is in feet above sea level.]

Location: $22 \cdot 31 \cdot 31.0411 \mathrm{FSL} .0190 \mathrm{FWL}$

Total depth: $\quad 1,465.0$ Land-surface altitude: $3,309.5$

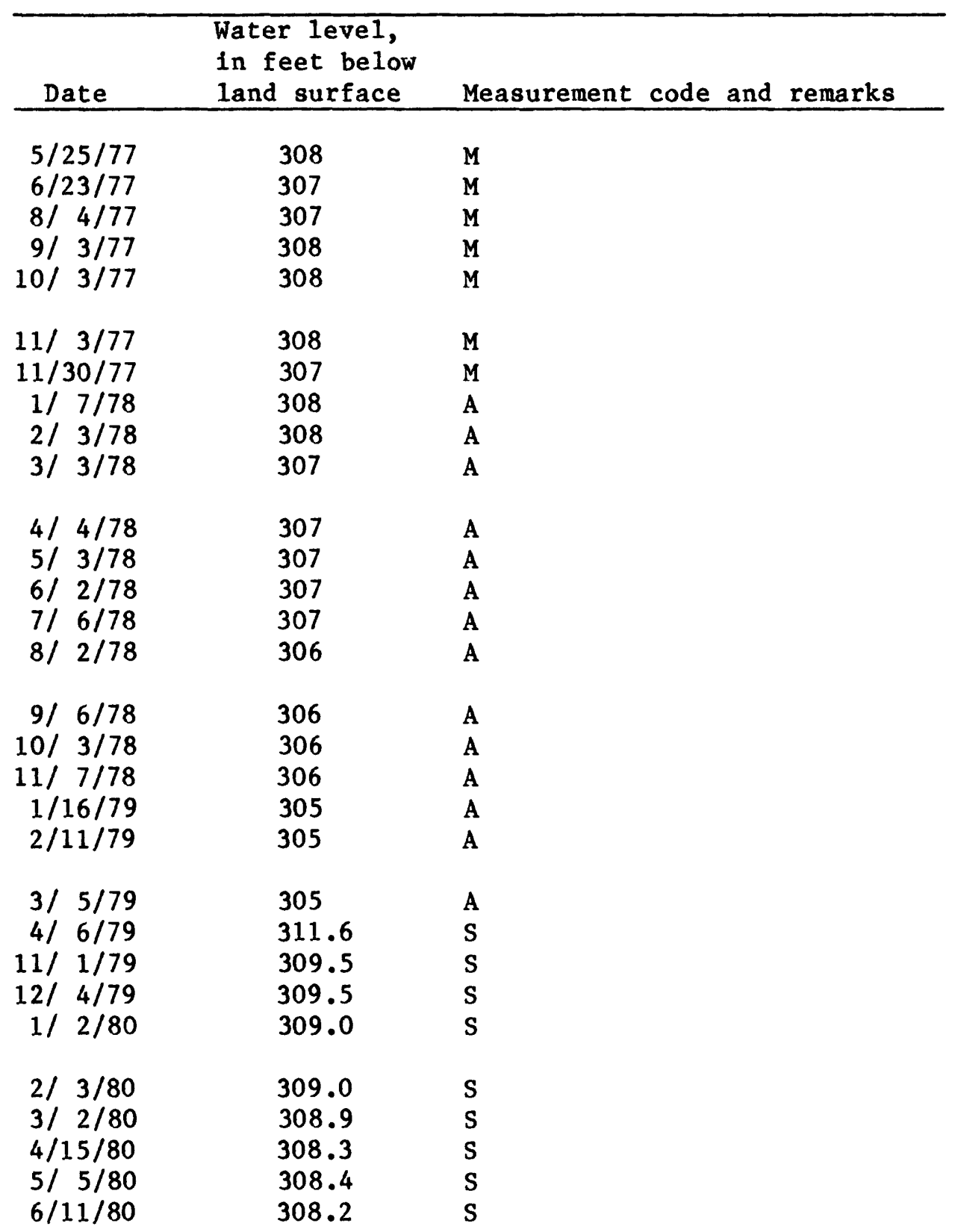


Table 38. Water-level records for well $\mathrm{P}-15$, Culebra Dolomite Member of the Rustler Formation - Concluded

\begin{tabular}{|c|c|c|}
\hline Date & $\begin{array}{l}\text { Water level, } \\
\text { in feet below } \\
\text { land surface }\end{array}$ & Measurement code and remarks \\
\hline 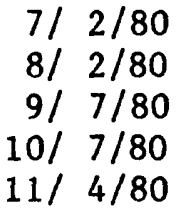 & $\begin{array}{l}308.3 \\
307.9 \\
307.9 \\
307.5 \\
307.1\end{array}$ & $\begin{array}{l}S \\
S \\
S \\
S \\
S\end{array}$ \\
\hline $\begin{aligned} 12 / & 4 / 80 \\
1 / & 5 / 81 \\
2 / & 4 / 81 \\
3 / & 5 / 81 \\
4 / & 2 / 81\end{aligned}$ & $\begin{array}{l}306.9 \\
306.6 \\
306.5 \\
306.2 \\
306.1\end{array}$ & $\begin{array}{l}S \\
S \\
S \\
S \\
S\end{array}$ \\
\hline $\begin{aligned} 5 / & 5 / 81 \\
6 / & 1 / 81 \\
7 / & 6 / 81 \\
10 / & 2 / 81 \\
11 / & 4 / 81\end{aligned}$ & $\begin{array}{l}305.9 \\
305.8 \\
306.0 \\
305.8 \\
305.4\end{array}$ & $\begin{array}{l}S \\
S \\
S \\
S \\
S\end{array}$ \\
\hline $\begin{array}{r}1 / 22 / 82 \\
5 / 25 / 82 \\
7 / 19 / 82 \\
9 / 8 / 82 \\
11 / 19 / 82\end{array}$ & $\begin{array}{l}305.2 \\
304.5 \\
304.2 \\
304.5 \\
304.2\end{array}$ & $\begin{array}{l}S \\
S \\
S \\
S \\
S\end{array}$ \\
\hline $\begin{array}{l}1 / 12 / 83 \\
8 / 25 / 83\end{array}$ & $\begin{array}{l}304.8 \\
304.9\end{array}$ & $\begin{array}{l}S \\
S\end{array}$ \\
\hline
\end{tabular}


Table 39. Water-level records for well P-15, Rustler FormationSalado Formation contact zone

[Measurement code: A, Air-line measurement; L, Lynes Pressure Sentry System; M, M-Scope; S, Steel tape; W, Winch. Total depth is in feet below land surface. Land-surface altitude is in feet above sea level.]

Location: 22.31.31.0411FSL.0190FWL

Total depth: $\quad 1,465.0$ Land-surface altitude: $\quad 3,309.5$

\begin{tabular}{|c|c|c|}
\hline Date & $\begin{array}{l}\text { Water level, } \\
\text { in feet below } \\
\text { land surface }\end{array}$ & Measurement code and remarks \\
\hline $\begin{array}{r}5 / 25 / 77 \\
6 / 23 / 77 \\
8 / 4 / 77 \\
9 / 4 / 77 \\
10 / 2 / 77\end{array}$ & $\begin{array}{l}341.7 \\
328.5 \\
328 \\
325 \\
327.4\end{array}$ & $\begin{array}{l}S \\
S \\
M \\
M \\
S\end{array}$ \\
\hline $\begin{array}{r}11 / 4 / 77 \\
11 / 30 / 77 \\
1 / 7 / 78 \\
2 / 3 / 78 \\
3 / 3 / 78\end{array}$ & $\begin{array}{l}327.2 \\
328.0 \\
328.6 \\
328.5 \\
328.5\end{array}$ & $\begin{array}{l}S \\
S \\
S \\
S \\
S\end{array}$ \\
\hline $\begin{array}{ll}4 / & 4 / 78 \\
4 / & 6 / 78 \\
5 / & 3 / 78 \\
6 / & 2 / 78 \\
7 / & 6 / 78\end{array}$ & $\begin{array}{l}323.1 \\
326.0 \\
322.2 \\
322.0 \\
322.1\end{array}$ & $\begin{array}{l}S \\
S \\
S \\
S \\
S\end{array}$ \\
\hline 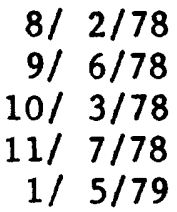 & $\begin{array}{l}323.1 \\
322.4 \\
320.4 \\
320.0 \\
318.7\end{array}$ & $\begin{array}{l}S \\
S \\
S \\
S \\
S\end{array}$ \\
\hline $\begin{array}{ll}2 / 10 / 79 \\
3 / & 5 / 79 \\
4 / & 6 / 79 \\
6 / 5 / 79 & 5 / 11 / 79 \\
7 / 11\end{array}$ & $\begin{array}{l}317.9 \\
316.9 \\
324.4 \\
371 \\
352.6\end{array}$ & $\begin{array}{l}S \\
S \\
S \\
M \\
S\end{array}$ \\
\hline $\begin{array}{rr}8 / & 6 / 79 \\
9 / & 6 / 79 \\
10 / & 9 / 79 \\
11 / & 1 / 79 \\
12 / & 4 / 79\end{array}$ & $\begin{array}{l}344.9 \\
348.3 \\
344.0 \\
339.2 \\
337.2\end{array}$ & $\begin{array}{l}S \\
S \\
S \\
S \\
S\end{array}$ \\
\hline
\end{tabular}


Table 39. Water-level records for well P-15, Rustler FormationSalado Formation contact zone - Concluded

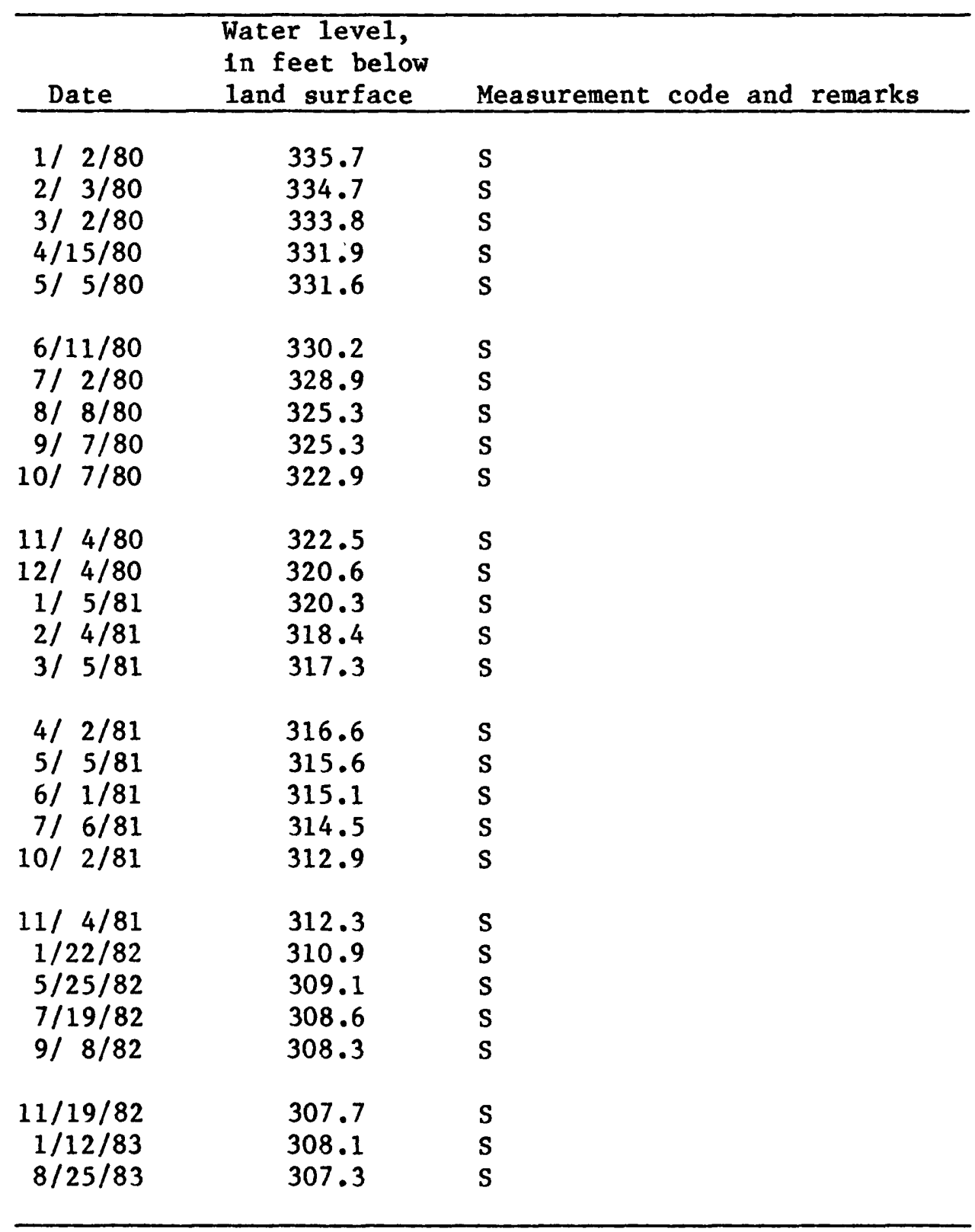


Table 40. Water-level records for well P-17, Culebra Dolomite Member of the Rustler Formation

[Measurement code: A, Alr-11ne measurement; L, Lynes Pressure Sentry System; M, M-Scope; S, Steel tape; W, Winch. Total depth is in feet below land surface. Land-surface altitude is in feet above sea level.]

Location: $23.31 .04 \cdot 1356 \mathrm{FSL} .0398 \mathrm{FWL}$

Total depth: $\quad 1,660.7$ Land-surface altitude: $3,334.2$

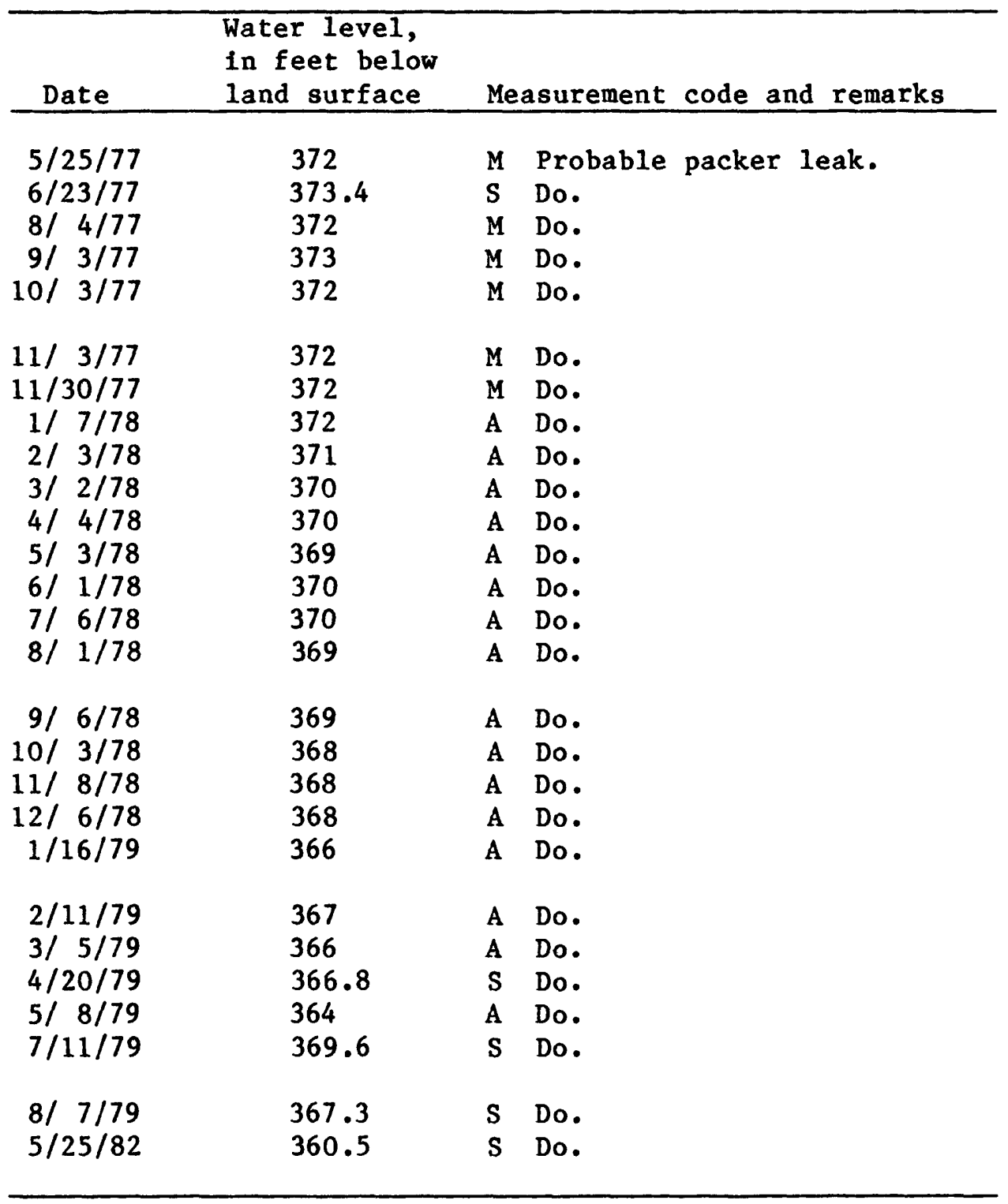


Table 41. Water-level records for well P-17, Rustler FormationSalado Formation contact zone

[Measurement code: A, Air-line measurement; L, Lynes Pressure Sentry System; M, M-Scope; S, Steel tape; W, Winch. Total depth is in feet below land surface. Land-surface altitude is in feet above sea level.]

Location: $23.31 .04 .1356 \mathrm{FSL} .0398 \mathrm{FWL}$

Total depth: 1,660.7 Land-surface altitude: $\quad 3,334.2$

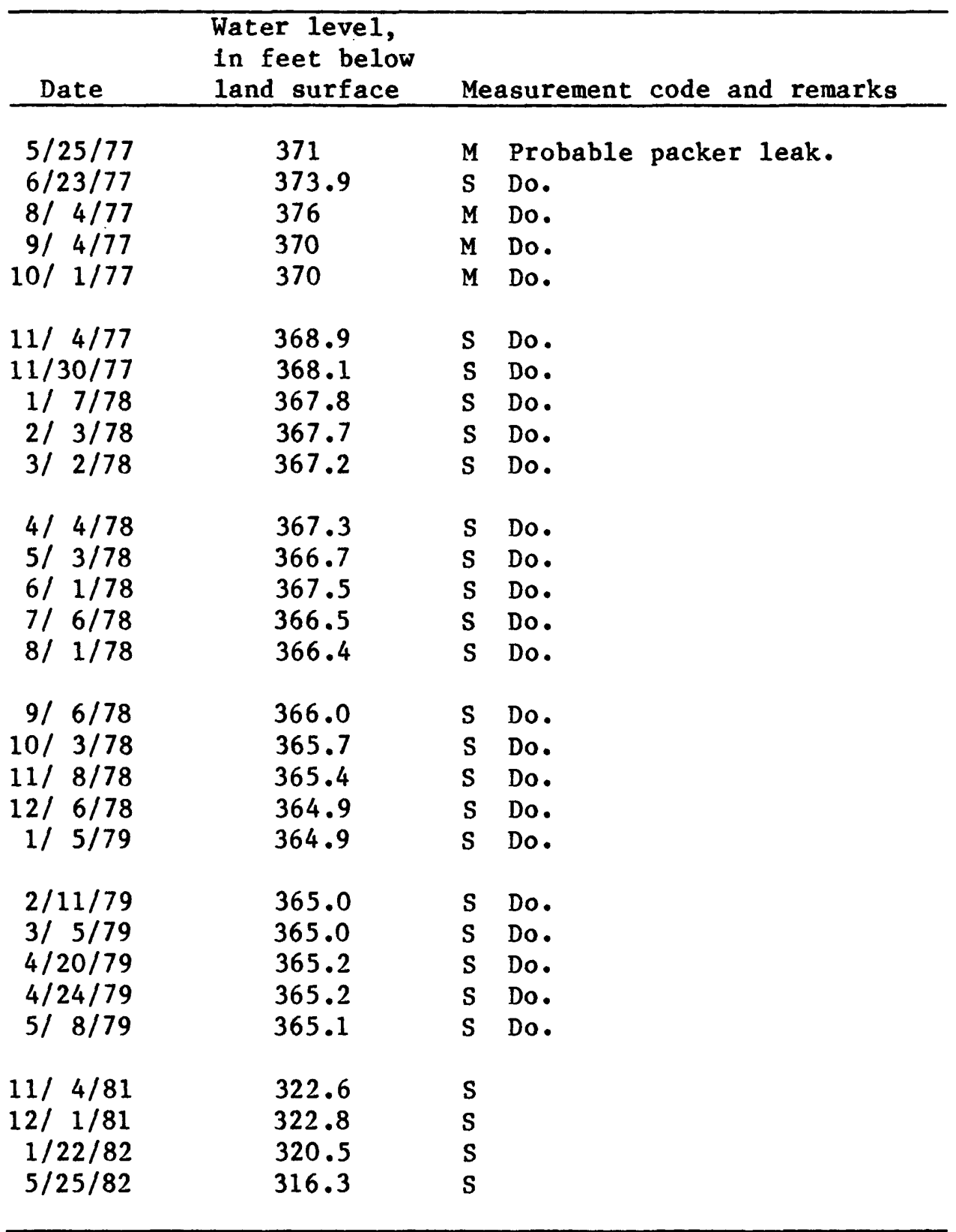


Table 42. Water-leve1 records for well P-18, Culebra Dolomite Member of the Rustler Formation

[Measurement code: A, Alr-1ine measurement; L, Lynes Pressure Sentry System; M, M-Scope; S, Steel tape; W, Winch. Total depth is in feet below land surface. Land-surface altitude is in feet above sea level.]

Location: $22.31 \cdot 26.0139 \mathrm{FSL} .0733 \mathrm{FEL}$

Total depth: $2,000.5$ Land-surface altitude: $\quad 3,475.8$

\begin{tabular}{|c|c|c|}
\hline Date & $\begin{array}{l}\text { Water level, } \\
\text { in feet below } \\
\text { land surface }\end{array}$ & Measurement code and remarks \\
\hline $\begin{array}{r}5 / 25 / 77 \\
8 / 4 / 77 \\
9 / 3 / 77 \\
10 / 3 / 77 \\
11 / 3 / 77\end{array}$ & $\begin{array}{r}1,034 \\
1,015 \\
1,016 \\
999 \\
993\end{array}$ & $\begin{array}{l}\text { A } \\
\text { A } \\
\text { A } \\
\text { A } \\
\text { A }\end{array}$ \\
\hline $\begin{array}{r}11 / 30 / 77 \\
1 / 7 / 78 \\
2 / 3 / 78 \\
3 / 2 / 78 \\
4 / 4 / 78\end{array}$ & $\begin{array}{l}986 \\
981 \\
972 \\
966 \\
958\end{array}$ & $\begin{array}{l}\text { A } \\
\text { A } \\
\text { A } \\
\text { A } \\
\text { A }\end{array}$ \\
\hline $\begin{array}{ll}5 / & 3 / 78 \\
6 / & 1 / 78 \\
7 / & 6 / 78 \\
8 / & 1 / 78 \\
9 / & 6 / 78\end{array}$ & $\begin{array}{l}951 \\
944 \\
934 \\
927 \\
917\end{array}$ & $\begin{array}{l}\text { A } \\
\text { A } \\
\text { A } \\
\text { A } \\
\text { A }\end{array}$ \\
\hline $\begin{array}{r}10 / 3 / 78 \\
11 / 8 / 78 \\
12 / 6 / 78 \\
1 / 16 / 79 \\
2 / 11 / 79\end{array}$ & $\begin{array}{l}907 \\
900 \\
895 \\
883 \\
878\end{array}$ & $\begin{array}{l}\text { A } \\
\text { A } \\
\text { A } \\
\text { A } \\
\text { A }\end{array}$ \\
\hline $\begin{array}{ll}3 / & 5 / 79 \\
5 / & 8 / 79 \\
5 / & 5 / 81 \\
5 / 11 / 81 \\
6 / 2 / 81\end{array}$ & $\begin{array}{l}874 \\
862 \\
765 \\
763 \\
759\end{array}$ & $\begin{array}{l}\text { A } \\
\text { A } \\
\text { A } \\
\text { A } \\
\text { A }\end{array}$ \\
\hline $\begin{array}{r}7 / 8 / 81 \\
8 / 4 / 81 \\
9 / 3 / 81 \\
10 / 1 / 81 \\
1 / 21 / 82\end{array}$ & $\begin{array}{l}753 \\
748 \\
744 \\
740 \\
714\end{array}$ & $\begin{array}{l}\text { A } \\
\text { A } \\
\text { A } \\
\text { A } \\
\text { A }\end{array}$ \\
\hline
\end{tabular}


Table 42. Water-leve1 records for well P-18, Culebra Dolomite Member of the Rustler Formation - Concluded

\begin{tabular}{|c|c|c|}
\hline Date & $\begin{array}{l}\text { Water level, } \\
\text { In feet below } \\
\text { land surface }\end{array}$ & Measurement code and remarks \\
\hline $\begin{array}{l}5 / 25 / 82 \\
8 / 19 / 82 \\
3 / 15 / 83\end{array}$ & $\begin{array}{l}713 \\
705 \\
703\end{array}$ & $\begin{array}{l}\text { A } \\
\text { A } \\
\text { W }\end{array}$ \\
\hline
\end{tabular}


Table 43. Water-level records for well P-18, Rustler FormationSalado Formation contact zone

[Measurement code: A, Air-1ine measurement; L, Lynes Pressure Sentry System; M, M-Scope; S, Steel tape; W, Winch. Total depth is in feet below land surface. Land-surface altitude is in feet above sea level.]

Location: $22.31 .26 .0139 \mathrm{FSL} .0733 \mathrm{FEL}$

Total depth: $\quad 2,000.5$ Land-surface altitude: $3,475.8$

\begin{tabular}{|c|c|c|}
\hline Date & $\begin{array}{l}\text { Water level, } \\
\text { in feet below } \\
\text { land surface }\end{array}$ & Measurement code and remarks \\
\hline $\begin{array}{r}5 / 25 / 77 \\
8 / 4 / 77 \\
9 / 4 / 77 \\
10 / 1 / 77 \\
11 / 4 / 77\end{array}$ & $\begin{array}{r}1,011 \\
882 \\
836 \\
801 \\
755\end{array}$ & $\begin{array}{l}M \\
M \\
M \\
M \\
M\end{array}$ \\
\hline $\begin{array}{r}11 / 30 / 77 \\
1 / 7 / 78 \\
2 / 3 / 78 \\
3 / 2 / 78 \\
4 / 4 / 78\end{array}$ & $\begin{array}{l}723 \\
676 \\
645 \\
612 \\
585\end{array}$ & $\begin{array}{l}\text { M } \\
\mathbf{A} \\
\mathbf{A} \\
\mathbf{A} \\
\mathbf{A}\end{array}$ \\
\hline $\begin{aligned} 5 / & 3 / 78 \\
6 / & 1 / 78 \\
7 / & 6 / 78 \\
10 / & 3 / 78 \\
11 / & 8 / 78\end{aligned}$ & $\begin{array}{l}557 \\
530 \\
503 \\
487 \\
472\end{array}$ & $\begin{array}{l}\mathbf{A} \\
\mathbf{A} \\
\mathbf{A} \\
\mathbf{A} \\
\mathbf{A}\end{array}$ \\
\hline 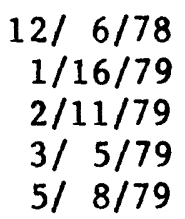 & $\begin{array}{l}461 \\
444 \\
435 \\
428 \\
409\end{array}$ & $\begin{array}{l}\mathbf{A} \\
\mathbf{A} \\
\mathbf{A} \\
\mathbf{A} \\
\mathbf{A}\end{array}$ \\
\hline $\begin{array}{ll}5 / & 5 / 81 \\
6 / & 2 / 81 \\
7 / & 8 / 81 \\
8 / & 4 / 81 \\
9 / & 3 / 81\end{array}$ & $\begin{array}{l}960 \\
921 \\
874 \\
840 \\
804\end{array}$ & $\begin{array}{l}W \\
W \\
W \\
W \\
W\end{array}$ \\
\hline $\begin{array}{r}10 / 1 / 81 \\
11 / 4 / 81 \\
12 / 1 / 81 \\
1 / 21 / 82 \\
2 / 18 / 82\end{array}$ & $\begin{array}{l}774 \\
737.6 \\
710.4 \\
665 \\
641.5\end{array}$ & $\begin{array}{l}W \\
S \\
S \\
W \\
S\end{array}$ \\
\hline
\end{tabular}


Table 43. Water-level records for well P-18, Rustler FormationSalado Formation contact zone - Concluded

\begin{tabular}{lcl}
\hline & $\begin{array}{l}\text { Water level, } \\
\text { in feet below } \\
\text { land surface }\end{array}$ & Measurement code and remarks \\
\hline $3 / 10 / 82$ & 626.7 & $\mathrm{~S}$ \\
$5 / 25 / 82$ & 574.9 & $\mathrm{~S}$ \\
$8 / 19 / 82$ & 527.9 & $\mathrm{~S}$ \\
$1 / 19 / 83$ & 624 & $\mathrm{~W}$ \\
\hline
\end{tabular}


Table 44. Water-level records for well WIPP-16, breccla

[Measurement code: A, Air-line measurement; L, Lynes Pressure Sentry System; M, M-Scope; S, Steel tape; W, Winch. Total depth is in feet below land surface. Land-surface altitude is in feet above sea level.]

Location: $21 \cdot 30.05 \cdot 2356 \mathrm{FSL}$.0139FWL

Total depth: $\quad 1,300.0$ Land-surface altitude: $\quad 3,383.0$

\begin{tabular}{ccc} 
& $\begin{array}{c}\text { Water level, } \\
\text { 1n feet below }\end{array}$ \\
Date & land surface & Measurement code and remarks \\
\hline $2 / 26 / 80$ & 282.7 & $\mathrm{~S}$ \\
$2 / 27 / 80$ & 282.9 & $\mathrm{~S}$ \\
$2 / 28 / 80$ & 283.0 & $\mathrm{~S}$ \\
$3 / 2 / 80$ & .284 .6 & $\mathrm{~S}$ \\
$3 / 6 / 80$ & 287.4 & $\mathrm{~S}$ \\
& & \\
$3 / 14 / 80$ & 291.6 & $\mathrm{~S}$ \\
$3 / 24 / 80$ & 294.7 & $\mathrm{~S}$ \\
$4 / 2 / 80$ & 296.5 & $\mathrm{~S}$ \\
$4 / 16 / 80$ & 298.7 & $\mathrm{~S}$ \\
$5 / 7 / 80$ & 300.8 & $\mathrm{~S}$ \\
& & \\
$6 / 9 / 80$ & 303.0 & $\mathrm{~S}$ \\
$6 / 30 / 80$ & 303.7 & $\mathrm{~S}$ \\
$10 / 8 / 80$ & 303.6 & $\mathrm{~S}$ \\
$11 / 3 / 80$ & 302.8 & $\mathrm{~S}$ \\
$12 / 1 / 80$ & 301.9 & $\mathrm{~S}$ \\
& & \\
$2 / 6 / 81$ & 299.6 & $\mathrm{~S}$ \\
$5 / 6 / 81$ & 297.4 & $\mathrm{~S}$ \\
$10 / 5 / 81$ & 294.5 & $\mathrm{~S}$ \\
$1 / 19 / 82$ & 293.5 & $\mathrm{~S}$ \\
& & \\
\hline
\end{tabular}


Table 45. Water-level records for well WIPP-25, Magenta Dolomite Member of the Rustler Formation

[Measurement code: A, Alr-1ine measurement; L, Lynes Pressure Sentry System; M, M-Scope; S, Steel tape; W, Winch. Total depth is in feet below land surface. Land-surface altitude is in feet above sea level.]

Location: 22.30.15.1853FSL.2838FEL

Total depth: $\quad 655.0$ Land-surface altitude: $\quad 3,211.8$

\begin{tabular}{rcc}
\hline & $\begin{array}{l}\text { Water level, } \\
\text { in feet below } \\
\text { Date }\end{array}$ & \\
\hline land surface & Measurement code and remarks \\
$10 / 8 / 80$ & 158 & $\mathrm{M}$ \\
$11 / 3 / 80$ & 157.8 & $\mathrm{~S}$ \\
$12 / 1 / 80$ & 158.3 & $\mathrm{~S}$ \\
$2 / 6 / 81$ & 158.6 & $\mathrm{~S}$ \\
$3 / 2 / 81$ & 158.6 & $\mathrm{~S}$ \\
& & \\
$5 / 7 / 81$ & 158.7 & $\mathrm{~S}$ \\
$10 / 5 / 81$ & 159.8 & $\mathrm{~S}$ \\
$1 / 19 / 82$ & 159.6 & $\mathrm{~S}$ \\
$5 / 18 / 82$ & 159.8 & $\mathrm{~S}$ \\
$7 / 19 / 82$ & 159.8 & $\mathrm{~S}$ \\
$9 / 8 / 82$ & 159.8 & $\mathrm{~S}$ \\
$11 / 19 / 82$ & 160.3 & $\mathrm{~S}$ \\
$1 / 12 / 83$ & 159.7 & $\mathrm{~S}$ \\
$8 / 24 / 83$ & 159.8 & $\mathrm{~S}$ \\
$9 / 29 / 83$ & 159.2 & $\mathrm{~S}$ \\
& & \\
$11 / 21 / 83$ & 158.6 & $\mathrm{~S}$ \\
$1 / 23 / 84$ & 158.3 & $\mathrm{~S}$ \\
$10 / 18 / 84$ & 157.7 & $\mathrm{~S}$ \\
$1 / 7 / 85$ & 157.2 & $\mathrm{~S}$ \\
& & \\
\hline
\end{tabular}


Table 46. Water-level records for well WIPP-25, Culebra Dolomite Member of the Rustler Formation

[Measurement code: A, Air-1ine measurement; L, Lynes Pressure Sentry System; M, M-Scope; S, Steel tape; W, Winch. Total depth is in feet below land surface. Land-surface altitude is in feet above sea level.]

Location: $22 \cdot 30 \cdot 15 \cdot 1853 \mathrm{FSL} .2838 \mathrm{FEL}$

Total depth: $\quad 655.0$ Land-surface altitude: $\quad 3,211.8$

\begin{tabular}{|c|c|c|}
\hline Date & $\begin{array}{l}\text { Water level, } \\
\text { in feet below } \\
\text { land surface }\end{array}$ & Measurement code and remarks \\
\hline $8 / 24 / 83$ & 160.4 & $\mathbf{s}$ \\
\hline $9 / 29 / 83$ & 160.4 & S \\
\hline $11 / 21 / 83$ & 160.5 & $\mathbf{S}$ \\
\hline $1 / 23 / 84$ & 161.3 & s \\
\hline $10 / 18 / 84$ & 159.7 & $\mathbf{s}$ \\
\hline $1 / 7 / 85$ & 161.0 & $\mathbf{s}$ \\
\hline
\end{tabular}


Table 47. Water-level records for well WIPP-25, Rustler FormationSalado Formation contact zone

[Measurement code: A, Afr-line measurement; L, Lynes Pressure Sentry System; M, M-Scope; S, Steel tape; W, Winch. Total depth is in feet below land surface. Land-surface altitude is in feet above sea level.]

Location: $22 \cdot 30 \cdot 15 \cdot 1853 \mathrm{FSL} .2838 \mathrm{FEL}$

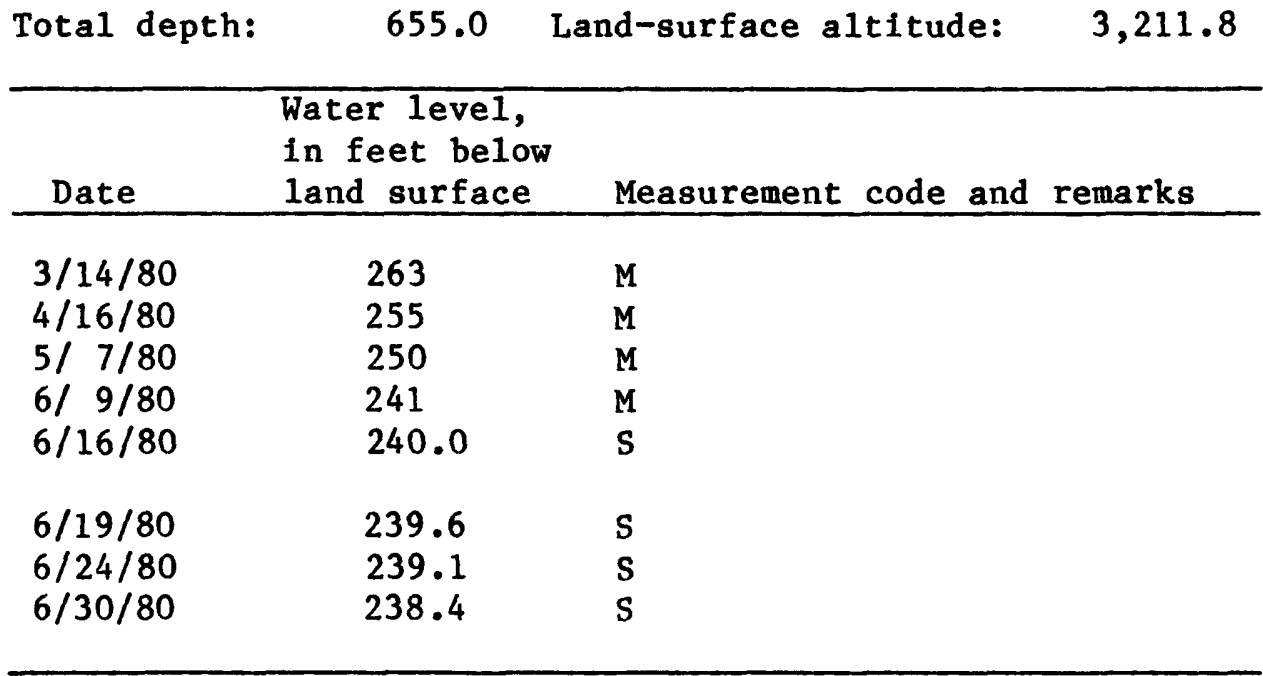


Table 48. Water-level records for well WIPP-26, Magenta Dolomite Member of the Rustler Formation

[Measurement code: A, Air-11ne measurement; L, Lynes Pressure Sentry System; M, M-Scope; S, Steel tape; W, Winch. Total depth is in feet below land surface. Land-surface altitude is in feet above sea leve1.]

Location: $22 \cdot 30 \cdot 29 \cdot 2232 \mathrm{FNL} .0012 \mathrm{FEL}$

Total depth: $\quad 503.0$ Land-surface altitude: $\quad 3,151.7$

\begin{tabular}{|c|c|c|}
\hline Date & $\begin{array}{l}\text { Water level, } \\
\text { in feet below } \\
\text { land surface }\end{array}$ & Measurement code and remarks \\
\hline $9 / 17 / 80$ & 127 & M \\
\hline $9 / 22 / 80$ & 127 & $M$ \\
\hline $10 / 8 / 80$ & 128 & M \\
\hline $11 / 3 / 80$ & 127.3 & $\mathbf{s}$ \\
\hline $12 / 1 / 80$ & 127.2 & s \\
\hline $3 / 4 / 81$ & 127.2 & $\mathbf{S}$ \\
\hline $5 / 7 / 81$ & 127.2 & S \\
\hline $1 / 19 / 82$ & 130.0 & S \\
\hline $5 / 19 / 82$ & 127.1 & $\mathbf{S}$ \\
\hline $7 / 19 / 82$ & 127.0 & $\mathbf{s}$ \\
\hline $9 / 8 / 82$ & 127.1 & $\mathbf{S}$ \\
\hline $11 / 19 / 82$ & 127.1 & s \\
\hline $1 / 12 / 83$ & 127.0 & $\mathbf{S}$ \\
\hline
\end{tabular}


Table 49. Water-1evel records for well WIPP-26, Culebra Dolomite Member of the Rustler Formation

[Measurement code: A, Alr-11ne measurement; L, Lynes Pressure Sentry System; M, M-Scope; S, Steel tape; W, Winch. Total depth is in feet below land surface. Land-surface altitude is in feet above sea level.]

Location: $22 \cdot 30 \cdot 29.2232 \mathrm{FNL} .0012 \mathrm{FEL}$

Total depth: $\quad 503.0$ Land-surface altitude: $\quad 3,151.7$

Water level,

in feet below

Date land surface

Measurement code and remarks

$8 / 24 / 83$

141.7

$S$

$9 / 29 / 83$

141.5

140.8

141.5

137.3

S

$1 / 24 / 84$

137.3

S

$10 / 20 / 84$

S 
Table 50. Water-level records for well WIPP-26, Rustler FormationSalado Formation contact zone

[Measurement code: A, Air-1ine measurement; L, Lynes Pressure Sentry System; M, M-Scope; S, Steel tape; W, Winch. Total depth is in feet below land surface. Land-surface altitude is in feet above sea level.]

Location: $22 \cdot 30 \cdot 29 \cdot 2232 \mathrm{FNL} .0012 \mathrm{FEL}$

Total depth: $\quad 503.0$ Land-surface altitude: $\quad 3,151.7$

\begin{tabular}{lcl}
\hline Date & $\begin{array}{l}\text { Water level, } \\
\text { in feet below } \\
\text { land surface }\end{array}$ & Measurement code and remarks \\
\hline $3 / 12 / 80$ & 217.0 & $\mathrm{~S}$ \\
$3 / 14 / 80$ & 201.3 & $\mathrm{~S}$ \\
$3 / 18 / 80$ & 197.6 & $\mathrm{~S}$ \\
$4 / 16 / 80$ & 195 & $\mathrm{M}$ \\
$5 / 7 / 80$ & 194 & $\mathrm{M}$ \\
$6 / 9 / 80$ & 192.1 & $\mathrm{~S}$ \\
$6 / 30 / 80$ & 191.7 & $\mathrm{~S}$ \\
\hline
\end{tabular}


Table 51. Water-level records for well WIPP-27, Magenta Dolomite Member of the Rustler Formation

[Measurement code: A, Air-line measurement; L, Lynes Pressure Sentry System; M, M-Scope; S, Steel tape; W, Winch. Total depth is in feet below land surface. Land-surface altitude is in feet above sea level.]

Location: $21 \cdot 30 \cdot 21.0090 \mathrm{FNL} \cdot 1485 \mathrm{FWL}$

Total depth: $\quad 592.0$ Land-surface altitude: $\quad 3,174.4$

\begin{tabular}{|c|c|c|}
\hline Date & $\begin{array}{l}\text { Water level, } \\
\text { in feet below } \\
\text { land surface }\end{array}$ & Measurement code and remarks \\
\hline $10 / 8 / 80$ & 106 & M \\
\hline $11 / 3 / 80$ & 104.1 & $S$ \\
\hline $12 / 1 / 80$ & 103.6 & S \\
\hline $2 / 6 / 81$ & 103.4 & S \\
\hline $3 / 4 / 81$ & 103.3 & S \\
\hline $5 / 6 / 81$ & 103.1 & $\mathrm{~s}$ \\
\hline $10 / 5 / 81$ & 102.1 & S \\
\hline $1 / 19 / 82$ & 102.2 & S \\
\hline $5 / 18 / 82$ & 103.5 & S \\
\hline $3 / 2 / 83$ & 105.6 & $\mathbf{S}$ \\
\hline $7 / 20 / 83$ & 107.3 & S \\
\hline $8 / 24 / 83$ & 107.5 & S \\
\hline $11 / 21 / 83$ & 107.0 & S \\
\hline $1 / 23 / 84$ & 106.9 & $\mathbf{S}$ \\
\hline $10 / 18 / 84$ & 106.4 & S \\
\hline $1 / 7 / 85$ & 106.5 & S \\
\hline
\end{tabular}


Table 52. Water-level records for well WIPP-27, Culebra Dolomite Member of the Rustler Formation

[Measurement code: A, Alr-1ine measurement; L, Lynes Pressure Sentry System; M, M-Scope; S, Steel tape; W, Winch. Total depth is in feet below land surface. Land-surface altitude is in feet above sea level.]

Location: $21 \cdot 30.21 .0090 \mathrm{FNL} \cdot 1485 \mathrm{FWL}$

\begin{tabular}{lll} 
Total depth: & 592.0 & Land-surface altitude: $3,174.4$ \\
\hline & $\begin{array}{l}\text { Water level, } \\
\text { 1n feet below } \\
\text { land surface }\end{array}$ & Measurement code and remarks \\
Date & 106.1 & $\mathrm{~S}$ \\
$8 / 24 / 83$ & 105.4 & $\mathrm{~S}$ \\
$11 / 21 / 83$ & 105.2 & $\mathrm{~S}$ \\
$1 / 23 / 84$ & 104.5 & $\mathrm{~S}$ \\
$10 / 18 / 84$ & 104.3 & $\mathrm{~S}$ \\
$1 / 7 / 85$ & & \\
\hline
\end{tabular}


Table 53. Water-level records for well WIPP-27, Rustler FormationSalado Formation contact zone

[Measurement code: A, Alr-1ine measurement; L, Lynes Pressure Sentry System; M, M-Scope; S, Steel tape; W, Winch. Total depth is in feet below land surface. Land-surface altitude is in feet above sea level.]

Location: $21 \cdot 30.21 .0090 \mathrm{FNL} .1485 \mathrm{FWL}$

Total depth: $\quad 592.0$ Land-surface altitude: $\quad 3,174.4$

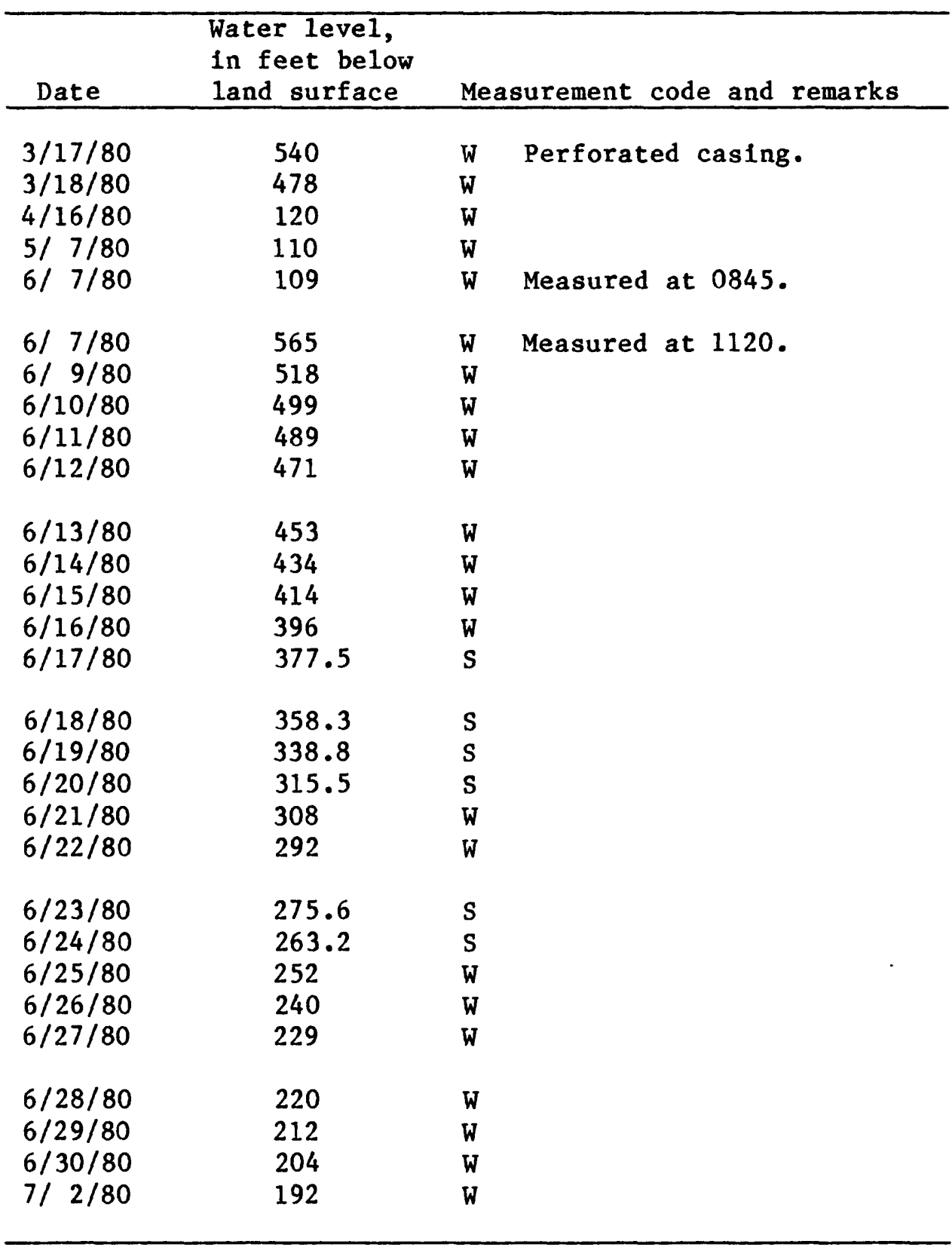


Table 54. Water-level records for well WIPP-28, Magenta Dolomite Member of the Rustler Formation

[Measurement code: A, Air-1ine measurement; L, Lynes Pressure Sentry System; M, M-Scope; S, Steel tape; W, Winch. Total depth is in feet below land surface. Land-surface altitude is in feet above sea level.]

Location: $21.31 \cdot 18.0099 \mathrm{FNL} .2401 \mathrm{FEL}$

Total depth: $\quad 801.0$ Land-surface altitude: $\quad 3,346.8$

\begin{tabular}{rcl}
\hline & $\begin{array}{c}\text { Water level, } \\
\text { 1n feet below } \\
\text { land surface }\end{array}$ & Measurement code and remarks \\
\hline Date & 340 & M \\
$9 / 23 / 80$ & 305 & M \\
$10 / 8 / 80$ & 232.8 & S \\
$11 / 3 / 80$ & 201.0 & S \\
$5 / 6 / 81$ & 201.7 & S \\
$7 / 7 / 81$ & 202.6 & S \\
$8 / 3 / 81$ & 202.9 & S \\
$9 / 1 / 81$ & 202.8 & S \\
$10 / 5 / 81$ & 202.4 & S \\
$1 / 19 / 82$ & 202.0 & S \\
$5 / 19 / 82$ & & \\
$3 / 2 / 83$ & 201.4 & S \\
\hline
\end{tabular}


Table 55. Water-level records for well WIPP-28, Culebra Dolomite Member of the Rustler Formation

[Measurement code: A, Air-line measurement; L, Lynes Pressure Sentry System; M, M-Scope; S, Steel tape; W, Winch. Total depth is in feet below land surface. Land-surface altitude is in feet above sea level.]

Location: $21.31 \cdot 18.0099 \mathrm{FNL} .2401 \mathrm{FEL}$

Total depth: $\quad 801.0$ Land-surface altitude: $\quad 3,346.8$

\begin{tabular}{|c|c|c|}
\hline Date & $\begin{array}{l}\text { Water level, } \\
\text { in feet below } \\
\text { land surface }\end{array}$ & Measurement code and remarks \\
\hline $9 / 29 / 83$ & 274.4 & s \\
\hline $11 / 21 / 83$ & 274.2 & s \\
\hline $1 / 23 / 84$ & 274.8 & S \\
\hline $10 / 18 / 84$ & 274.6 & $\mathbf{S}$ \\
\hline $1 / 7 / 85$ & 274.2 & S \\
\hline
\end{tabular}


Table 56. Water-level records for well WIPP-28, Rustler FormationSalado Formation contact zone

[Measurement code: A, Air-1ine measurement; L, Lynes Pressure Sentry System; M, M-Scope; S, Steel tape; W, WInch. Total depth is in feet below land surface. Land-surface altitude is in feet above sea level.]

Location: $21 \cdot 31.18 .0099 \mathrm{FNL} .2401 \mathrm{FEL}$

Total depth: $\quad 801.0$ Land-surface altitude: $\quad 3,346.8$

\begin{tabular}{lcl}
\hline & $\begin{array}{l}\text { Water level, } \\
\text { In feet below } \\
\text { land surface }\end{array}$ & Measurement code and remarks \\
\hline $3 / 15 / 80$ & 410 & W \\
$3 / 17 / 80$ & 330 & W \\
$4 / 16 / 80$ & 314 & W \\
$5 / 7 / 80$ & 308 & W \\
$6 / 9 / 80$ & 304 & W \\
& & \\
$6 / 19 / 80$ & 303.1 & $\mathrm{~S}$ \\
$6 / 24 / 80$ & 303.3 & $\mathrm{~S}$ \\
$6 / 30 / 80$ & 303.3 & $\mathrm{~S}$ \\
$8 / 24 / 83$ & 295.4 & $\mathrm{~S}$ \\
$9 / 29 / 83$ & 294.1 & $\mathrm{~S}$ \\
& & \\
$11 / 21 / 83$ & 294.2 & $\mathrm{~S}$ \\
$1 / 23 / 84$ & 293.9 & $\mathrm{~S}$ \\
$10 / 18 / 84$ & 293.0 & $\mathrm{~S}$ \\
$1 / 7 / 85$ & 291.8 & $\mathrm{~S}$ \\
& & \\
\hline
\end{tabular}


Table 57. Water-level records for we11 WIPP-29, Culebra Dolonite Member of the Rustler Formation

[Measurement code: A, Afr-1ine measurement; L, Lynes Pressure Sentry System; M, M-Scope; S, Steel tape; W, Winch. Total depth is in feet below land surface. Land-surface altitude is in feet above sea level.]

Location: $22 \cdot 29.34 .0407 \mathrm{FSL} .1828 \mathrm{FEL}$

Total depth: $\quad 376.0$ Land-surface altitude: $2,977.1$

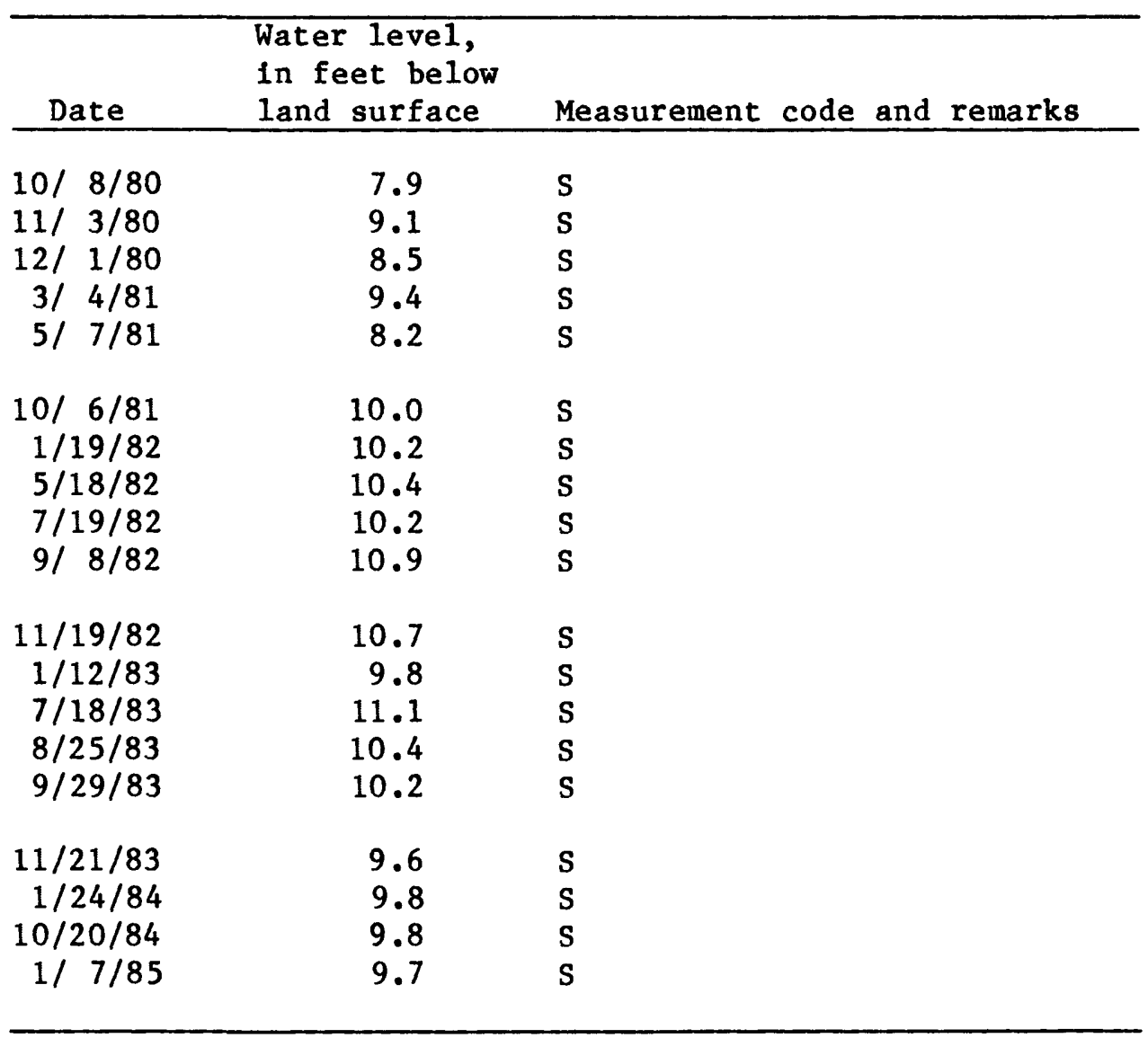


Table 58. Water-level records for well WIPP-29, Rustler FormationSalado Formation contact zone

[Measurement code: A, Alr-line measurement; L, Lynes Pressure Sentry System; M, M-Scope; S, Steel tape; W, Winch. Total depth is in feet below land surface. Land-surface altitude is in feet above sea level.]

Location: $22 \cdot 29 \cdot 34 \cdot 0407$ FSL.1828FEL
Total depth: 376.0
Land-surface altitude:
$2,977.1$

\begin{tabular}{|c|c|c|}
\hline Date & $\begin{array}{l}\text { Water level, } \\
\text { in feet below } \\
\text { land surface }\end{array}$ & Measurement code and remarks \\
\hline $\begin{array}{l}3 / 11 / 80 \\
3 / 14 / 80 \\
4 / 15 / 80 \\
5 / 7 / 80 \\
6 / 9 / 80\end{array}$ & $\begin{array}{l}20.5 \\
19.1 \\
19.2 \\
19.2 \\
17.0\end{array}$ & $\begin{array}{l}S \\
S \\
S \\
S \\
S\end{array}$ \\
\hline $\begin{array}{r}6 / 30 / 80 \\
8 / 25 / 83 \\
9 / 29 / 83 \\
11 / 21 / 83 \\
1 / 24 / 84\end{array}$ & $\begin{array}{l}17.6 \\
39.0 \\
38.4 \\
37.1 \\
36.4\end{array}$ & $\begin{array}{l}S \\
S \\
S \\
S \\
S\end{array}$ \\
\hline $\begin{array}{r}10 / 20 / 84 \\
1 / 7 / 85\end{array}$ & $\begin{array}{l}31.1 \\
30.0\end{array}$ & $\begin{array}{l}S \\
S\end{array}$ \\
\hline
\end{tabular}


Table 59. Water-level records for well WIPP-30, Magenta Dolomite Member of the Rustler Formation

[Measurement code: A, Air-line measurement; L, Lynes Pressure Sentry System; M, M-Scope; S, Steel tape; W, Winch. Total depth is in feet below land surface. Land-surface altitude is in feet above sea level.]

Location: $21 \cdot 31 \cdot 33.0668 \mathrm{FNL} .0177 \mathrm{FWL}$

Total depth: $\quad 913.0$ Land-surface altitude: $\quad 3,424.8$

\begin{tabular}{rcc}
\hline & $\begin{array}{l}\text { Water level, } \\
\text { 1n feet below }\end{array}$ \\
Date & land surface & Measurement code and remarks \\
\hline $9 / 22 / 80$ & 419 & $\mathrm{M}$ \\
$9 / 23 / 80$ & 411 & $\mathrm{M}$ \\
$9 / 24 / 80$ & 403 & $\mathrm{M}$ \\
$12 / 1 / 80$ & 303.7 & $\mathrm{~S}$ \\
$5 / 7 / 81$ & 316 & $\mathrm{M}$ \\
& & \\
$10 / 7 / 81$ & 308 & $\mathrm{M}$ \\
$1 / 19 / 82$ & 306 & $\mathrm{M}$ \\
$5 / 19 / 82$ & 304.0 & $\mathrm{~S}$ \\
$7 / 19 / 82$ & 303.9 & $\mathrm{~S}$ \\
$9 / 8 / 82$ & 303.9 & $\mathrm{~S}$ \\
& & \\
$11 / 19 / 82$ & 303.6 & $\mathrm{~S}$ \\
$1 / 12 / 83$ & 303.4 & $\mathrm{~S}$ \\
$7 / 21 / 83$ & 302.9 & $\mathrm{~S}$ \\
$8 / 23 / 83$ & 336.7 & $\mathrm{~S}$ \\
$9 / 29 / 83$ & 335.6 & $\mathrm{~S}$ \\
$11 / 21 / 83$ & 335.3 & $\mathrm{~S}$ \\
$1 / 23 / 84$ & 335.1 & $\mathrm{~S}$ \\
$10 / 18 / 84$ & 333.4 & $\mathrm{~S}$ \\
$1 / 7 / 85$ & 333.3 & $\mathrm{~S}$ \\
& & \\
\hline
\end{tabular}


Table 60. Water-1evel records for well WIPP-30, Culebra Dolomite Member of the Rustler Formation

[Measurement code: A, Alr-line measurement; L, Lynes Pressure Sentry System; M, M-Scope; S, Steel tape; W, Winch. Total depth is in feet below land surface. Land-surface altitude is in feet above sea level.]

Location: $21.31 \cdot 33.0668 \mathrm{FNL} .0177 \mathrm{FWL}$

Total depth: $\quad 913.0$ Land-surface altitude: $\quad 3,424.8$

\begin{tabular}{|c|c|c|}
\hline Date & $\begin{array}{l}\text { Water level, } \\
\text { in feet below } \\
\text { land surface }\end{array}$ & Measurement code and remarks \\
\hline $\begin{array}{r}8 / 23 / 83 \\
9 / 29 / 83 \\
11 / 21 / 83 \\
1 / 23 / 84 \\
10 / 18 / 84\end{array}$ & $\begin{array}{l}401.5 \\
400.3 \\
398.7 \\
397.0 \\
386.9\end{array}$ & $\begin{array}{l}S \\
S \\
S \\
S \\
S\end{array}$ \\
\hline $1 / 7 / 85$ & 386.4 & $S$ \\
\hline
\end{tabular}


Table 61. Water-level records for well WIPP-30, Rustler FormationSalado Formation contact zone

[Measurement code: A, Air-1ine measurement; L, Lynes Pressure Sentry System; M, M-Scope; S, Steel tape; W, Winch. Total depth is in feet below land surface. Land-surface altitude is in feet above sea level.]

Location: 21.31 .33 .0668 FNL.0177FWL

Total depth: $\quad 913.0$ Land-surface altitude: $\quad 3,424.8$

\begin{tabular}{|c|c|c|}
\hline Date & $\begin{array}{l}\text { Water level, } \\
\text { in feet below } \\
\text { land surface }\end{array}$ & Measurement code and remarks \\
\hline $\begin{array}{l}4 / 15 / 80 \\
5 / 7 / 80 \\
6 / 9 / 80 \\
6 / 16 / 80 \\
6 / 19 / 80\end{array}$ & $\begin{array}{l}474 \\
474 \\
473 \\
473.2 \\
473.2\end{array}$ & $\begin{array}{l}W \\
W \\
W \\
S \\
S\end{array}$ \\
\hline $\begin{array}{l}6 / 24 / 80 \\
6 / 30 / 80\end{array}$ & $\begin{array}{l}473.3 \\
473.3\end{array}$ & $\begin{array}{l}S \\
S\end{array}$ \\
\hline
\end{tabular}

